\title{
An Investigation into \\ Clustering \& \\ Segregation \\ in Granular Materials
}

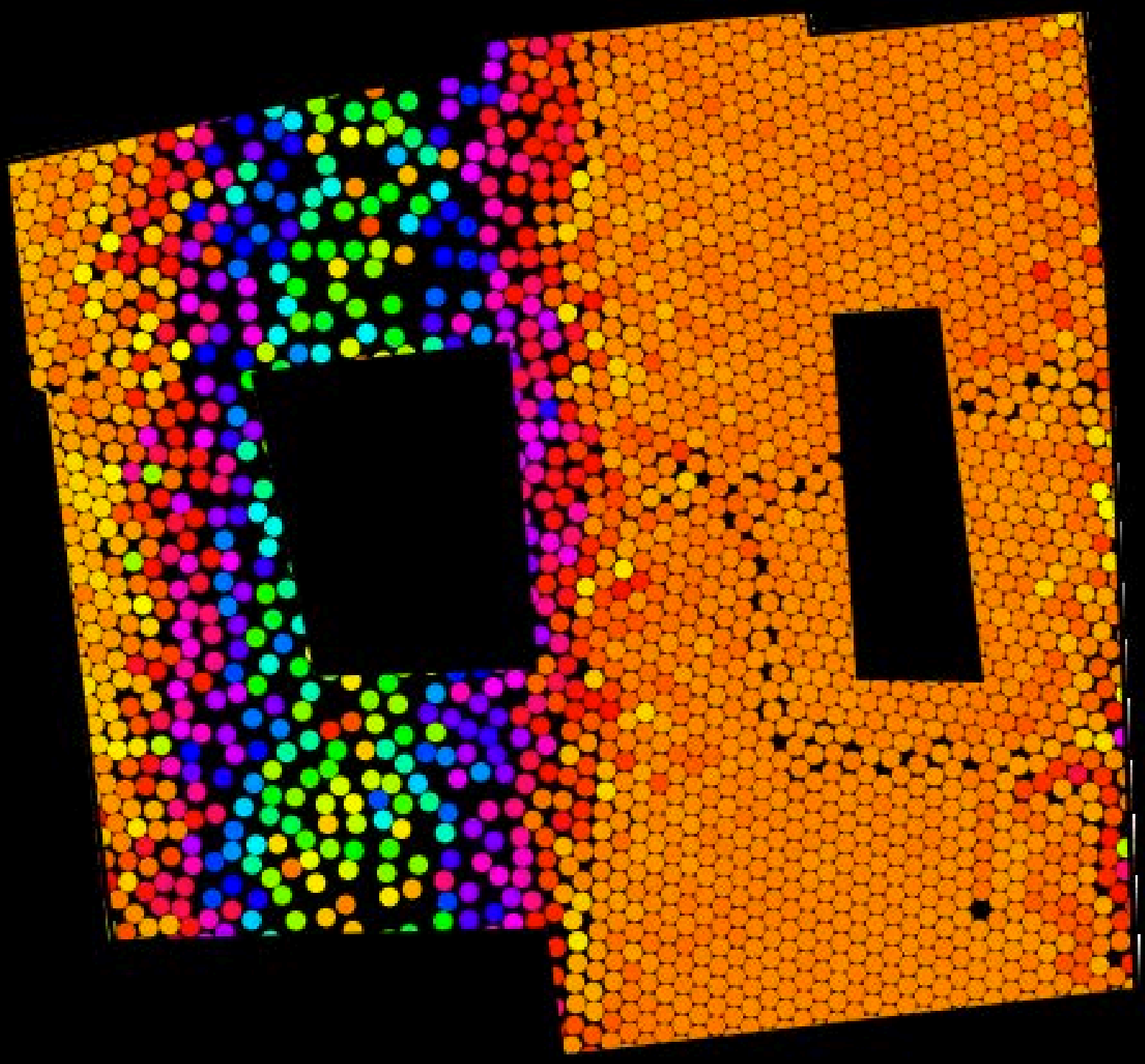

Sebastián González 


\title{
An Investigation into Clustering and Segregation in Granular Materials
}

\author{
Juan Sebastián Lisardo González Briones
}



Nec spe nec metu 


\section{Samenstelling promotiecommissie:}

Prof. dr. F. Eising (voorzitter)

Prof. dr. S. Luding (promotor)

Dr. A.R. Thornton (assistent promotor)

Prof. dr. D. van de Meer

Dr. ir. M. van der hoef

Prof. dr. T. Poeschel

Dr. ir. J.R. van Ommen

Dr. I. Einav

Dr. R. Soto
Univeristy of Twente Univeristy of Twente Univeristy of Twente Univeristy of Twente Univeristy of Twente Universität Erlangen-Nürnberg Delft Univerity of Technology University of Sydney Universidad de Chile

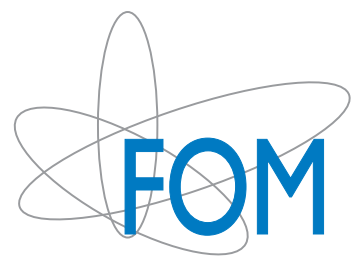

Dit werk maakt deel uit van het onderzoekprogramma van de Stichting voor Fundamenteel Onderzoek der Materie (FOM), die deel uit maakt van de Nederlandse Organisatie voor Wetenschappelijk Onderzoek (NWO).

ISBN : 978-90-365-3540-3

DOI nummer : 10.3990/1.9789036535403

Officiële URL: http://dx.doi.org/10.3990/1.9789036535403

This work (included the cover image) is licensed under the Creative Commons Attribution 3.0 Unported License. To view a copy of this license, visit http: //creativecommons.org/licenses/by/3.0/ or send a letter to Creative Commons, 444 Castro Street, Suite 900, Mountain View, California, 94041, USA.

\section{Cccreative}




\title{
An Investigation into Clustering and Segregation in Granular Materials
}

\author{
PROEFSCHRIFT
}

ter verkrijging van

de graad van doctor aan de Universiteit Twente, op gezag van de rector magnificus,

Prof. dr. H. Brinksma,

volgens besluit van het College voor Promoties in het openbare te verdedigen

om vrijdag 19 april 2013 om 14:45 uur

door

Juan Sebastián Lisardo González Briones geboren op 29 juli 1983

te Santiago, Chili 
Dit proefschrift is goedgekeurd door de promotor prof. dr. S. Luding en de assistent promotor dr. A.R. Thornton 


\section{Overview}

Granular matter is ubiquitous in our daily life yet far from completely understood. The aim of this thesis is to investigate extensions to and validation of simple granular models, for systems with either long-range force or particle size distributions. Electrostatic interactions due to tribo-charging, van der Waals forces in nano-particles, size-driven segregation in granular flows; all these phenomena necessitate going beyond the simple mono-sized short-range interaction model usually used to study granular matter.

The first chapter gives a general introduction to the themes and topics treated in this thesis. In the second chapter I study the differences in the stability properties of the Homogeneous Cooling State (HCS) of a twodimensional mono-disperse collection of rigid and near-elastic disks, obtained by using different advanced formulae for the pair-correlation function at high densities. I found out that for the modified pair correlation function at contact gives a better estimate for the energy dissipation and predicts the existence of new instable hydrodynamic modes. The third chapter presents the results of my extension to event-driven simulations in order to deal with sticky particles, as a way of mimicking van der Waals forces at play in nanoaerosols. I developed a new cluster-based event-driven algorithm that allows the simulation of cluster formation in a two dimensional gas: particles move freely until they collide and stick together irreversibly. These clusters aggregate into bigger structures in an isotropic and random way, forming fractal structures whose fractal dimension depends on the initial density of the system. The fourth chapter focuses on the phase diagram of granular cooling with long range interactions. I analyze structure formation in this system by means of two methods: molecular dynamics with discrete potentials and linear stability analysis of the corresponding hydrodynamic equations. For low dissipation and density, the data is exactly fitted by a previous mean field theory developed for continuous potentials which is quite remarkable and supports the use of discrete potentials and event-driven methods. The fifth chapter presents a new way to control axial segregation in rotating tumblers, which consist in making the tumbler non-homogeneous in the axial direction. I explain how this happens and the conditions for it. The sixth chapter is an essay on how I want to do science and the problems I see nowadays with it. Finally, conclusions and outlook close the thesis. 



\section{Sammenvatting}

Hoewel granulaire materie overal om ons heen aanwezig is, is de werking van de onderliggende fysische processen nog lang niet begrepen. Het doel van dit proefschrift is om de uitbreidingen en de validatie van eenvoudige granulaire modellen te onderzoeken voor systemen met lange-afstands krachten of met deeltjes die van grootte verschillen. Elektrostatische interacties door tribologische oplading, vanderwaalskrachten in nanodeeltjes, grootte gedreven segregatie in granulaire stromingen; al deze verschijnselen maken het noodzakelijk modellen te onwikkelen die complexer zijn dan de eenvoudige homogene grootte en korte afstands interactiemodellen die meestal gebruikt worden om granulaire materie te bestuderen. Het eerste hoofdstuk geeft een algemene inleiding op de thema's en onderwerpen behandeld in dit proefschrift. In het tweede hoofdstuk bestuderen we de verschillen in de stabiliteitseigenschappen van de homogene koelings toestand van een tweedimensionale, monodisperse verzameling van stijve en vrijwel elastische schijven, verkregen door het gebruiken van verschillende geavanceerde vergelijkingen voor paar-correlatie functies bij hoge dichtheden. Het derde hoofdstuk presenteert de resultaten van mijn modificatie op event-driven simulaties om cohesieve deeltjes te integreren, een manier om vanderwaalskrachten te modeleren in nano-aerosols. Ik heb een nieuw cluster-based event-driven algoritme ontwikkeld dat de simulatie mogelijk maakt van cluster formatie in een tweedimensionaal gas: deeltjes bewegen vrij tot dat deze botsen en onomkeerbaar samen kleven. Deze clusters vormen samen grotere structuren op een isotropische en willekeurige manier, om structuren te vormen wiens fractale dimensies afhangen van de initiele dichtheid van het systeem. Het vierde hoofdstuk richt zich op het fasediagram van granulaire koelprocessen met lange afstand interacties. Ik analyseer structuur formatie in dit systeem door gebruik te maken van twee methodes: moleculaire dynamica met discrete potentialen en lineaire stabiliteits analyse van de overeenkomende hydrodynamische vergelijkingen. Voor lage dissipatie en dichtheid komen de data precies overeen met een eerder ontwikkelde mean-field theorie voor continue potentialen. Het vijfde hoofdstuk presenteert een nieuwe manier om axiale segregatie in draaiende trommels te beïnloeden. Dit wordt bereikt door de trommel inhomogeen te maken in de axiale richting. We verklaren hoe dit gebeurt en onder welke condities. Het zesde hoofdstuk is een opstel over hoe ik aan de wetenschap wil bijdragen en de problemen van tegenwoordig zoals ik ze zie. Tot slot eindig ik dit proefschrift met conclusies en vooruitzichten. 



\section{Acknowledgements}

One is used to say "I'm doing my PhD...", like if the thesis would belong to one, or the PhD would be one's posession. I don't think so. This work, and more generally myself, wouldn't exist (or at least not in the present form) if it weren't for the help and support of many people. Friends, family and colleagues, they all contributed to make the last four years what they were, and in one way or another, they wrote with me this thesis.

In no particular order, then, I would like to thanks: Yasaman for her friendship, smile and dinners; Nico for all the talks, bottles and books we shared; Chanse for the music, the arguments, and the light he brought to my life; Willem for giving me a home and a life lasting friendship; Fela for just being there, always; Samie for his stories and his utter defense of the right to enjoy; Sadra and Pauli for the photos of the experiment and friendship; SinQuenza for bringing art, music and Dutch into my life; Marcelo for all the mails, the trips and BBQ's; Natalia, for giving me the first push to come here, and all her support; to my father, for teaching me that knowledge is the only thing one cannot part with; to my mother, for all the love; Pelao and Jaru for the friendship in the distance and the closeness; Mycke for the guitar and the translation; Gaspar, for all the words and his help; Stefan, my advisor, for the freedom, the rigor and the trust he gave me; Ant for his support and all the jokes; Sylvia, for bearing me all this time and for making the office a place I want to go; to Julia, for having the courage to stay with me and the patience to wait while I was finishing my thesis. Without you all, nothing would be possible, thanks again. 



\section{Contents}

1 Introduction $\quad 1$

1.1 Granular Matter . . . . . . . . . . . . . . . . . . . 2

1.1.1 Granular Gases . . . . . . . . . . . . . . . . 3

1.1.2 Granular Flow in Rotating Tumblers . . . . . . . . . . 4

1.2 Simulations . . . . . . . . . . . . . . . . 4

1.2.1 Event-Driven Molecular Dynamics . . . . . . . . . 5

1.2.2 Discrete Element Method . . . . . . . . . . . . . 6

1.3 Thesis Structure . . . . . . . . . . . . . . . . . 7

2 Cooling of dense 2D hard-spheres gases 9

2.1 Introduction . . . . . . . . . . . . . . . . . . . 9

2.2 From homogeneous to inhomogeneous cooling . . . . . . . . . 10

2.2.1 Collision Model . . . . . . . . . . . . . . . . 11

2.2.2 Free Cooling Granular Gas . . . . . . . . . . . . . . . . . 11

2.3 Hydrodynamics . . . . . . . . . . . . . . . . . . . . . 12

2.3.1 Mass balance . . . . . . . . . . . . . . 12

2.3.2 Momentum balance . . . . . . . . . . . . . 12

2.3.3 Energy balance . . . . . . . . . . . . . . 13

2.3.4 The ("classical") transport coefficients . . . . . . . 13

2.4 Stability Analysis . . . . . . . . . . . . . . . . . . . 18

2.4.1 Collision rate . . . . . . . . . . . . . . . 18

2.4 .2 Linear equations . . . . . . . . . . . . . . . . . . 18

2.4 .3 Shear instability . . . . . . . . . . . . . 20

2.4.4 Heat and sound instabilities . . . . . . . . . . 20

2.4.5 Numerical Simulations . . . . . . . . . . . . . 23

2.5 Conclusions . . . . . . . . . . . . . . . . . . . 25

3 An Event-Driven Algorithm for Fractal Cluster Formation 29

3.1 Introduction . . . . . . . . . . . . . . . . . . . . 29

3.2 Algorithm . . . . . . . . . . . . . . . . . . . . . . . . . . . . . . . . . . .

3.3 Simulations . . . . . . . . . . . . . . . . . . . . 32

3.3.1 Temporal Evolution . . . . . . . . . . . . 33

3.3.2 Cluster size distribution . . . . . . . . . . . . . 33

3.3.3 Fractal dimension and density . . . . . . . . . . . 34

3.4 Conclusions . . . . . . . . . . . . . . . . . . . 35 
4 Free Cooling of Hard-Spheres with Long-range Interactions 39

4.1 Introduction . . . . . . . . . . . . . . . . . . . . 39

4.2 Classical Granular Gas . . . . . . . . . . . . . . . 40

4.2.1 Free Cooling Theory . . . . . . . . . . . . . . 41

4.2.2 Hydrodynamic Equations . . . . . . . . . . . . . 41

4.2 .3 Cluster Instability . . . . . . . . . . . . . . . . 42

4.3 Long-range cooling: modification of the dissipation rate . . . . 43

4.4 Stability Analysis . . . . . . . . . . . . . . . . . . . 44

4.5 Event-Driven simulations . . . . . . . . . . . . . . . . . . 48

4.5.1 Discontinuous potentials . . . . . . . . . . . . 49

4.5.2 System and Preparation . . . . . . . . . . . . . 51

4.6 Numerical Results . . . . . . . . . . . . . . . . . . . . . . 51

4.6.1 Attractive forces . . . . . . . . . . . . . . . . 51

4.6.2 Repulsive forces . . . . . . . . . . . . . . . . 58

4.7 Phase diagram for cooling with long-range interactions . . . . 62

4.8 Conclusions . . . . . . . . . . . . . . . . 63

$\begin{array}{lll}5 & \text { Shaping Segregation } & 67\end{array}$

5.1 Introduction . . . . . . . . . . . . . . . . . 67

5.2 Experimental set-up . . . . . . . . . . . . . . . . 68

5.3 The effect of the filling fraction . . . . . . . . . . . 70

5.4 Bi-stable flow . . . . . . . . . . . . . . . . . 70

5.5 Comparison of Star with Pentagon . . . . . . . . . . 71

5.6 Shape-induced axial segregation . . . . . . . . . . . . 76

5.7 Conclusions . . . . . . . . . . . . . . . . . . 78

6 A ritual that is not rotten $\quad 81$

6.1 Introduction . . . . . . . . . . . . . . . . . 81

6.2 The Beginnings . . . . . . . . . . . . . . . . . . . 82

6.3 Genealogy of Science . . . . . . . . . . . . . . . . 85

6.4 The University Today . . . . . . . . . . . . . . . . . . . 86

6.5 What science can say . . . . . . . . . . . . . . . 86

6.6 Philosophical Position . . . . . . . . . . . . . . 87

6.7 What is to be done? . . . . . . . . . . . . . . 90 90

6.8 A ritual that is not rotten . . . . . . . . . . . . . . . 92

6.9 Conclusion: The science I want to make . . . . . . . . . . 93

7 Conclusions and Outlook $\quad 95$ 


\section{Chapter 1}

\section{Introduction}

Spheres have fascinated humanity since its origins; as Borges tell us [1] - and deserves to be quoted at length -

Six centuries before the Christian era, the rhapsodist Xenophanes of Colophon, wearied of the Homeric verses he recited from city to city, lashed out at the poets who attributed anthropomorphic traits to the gods, and offered the Greeks a single God, a god who was an eternal sphere. In the Timaeus of Plato we read that the sphere is the most perfect and most uniform figure, for all points of its surface are equidistant from its centre; Olof Gigon (Ursprung de griechischen Philosophie, 183) understands Xenophanes to speak analogically: God is spherical because that form is best - or least inadequate - to represent the Divinity. Parmenides, forty years later, rephrased the image: "The Divine Being is like the mass of a well-rounded sphere, whose force is constant from the center in any direction." Calogero and Mondolfo reasoned that Parmenides intuited an infinite, or infinitely expanding sphere, and that the words just transcribed possess a dynamic meaning (Albertelli: Gli Eleati, 148). Parmenides taught in Italy; a few years after his death, the Sicilian Empedocles of Agrigentum constructed a laborious cosmology: a stage exists in which the particles of earth, water, air and fire make up a sphere without end, "the rounded Sphairos, which exults in its circular solitude."

It may be that universal history is the history of the different intonations given a handful of metaphors.

The aim of this $\mathrm{PhD}$ thesis is to yet again enunciate a handful of metaphors concerning spheres with some of the simplest possible interaction models. 
We will focus on the study of granular matter through simulations. In practice, this means that the spheres we will consider are governed by two physical principles: excluded volume and dissipation. There are several ways of implementing such behavior. We focus on two of them: (a) the discrete particle method (DPM), where for a given model of the material the interactions forces are computed and numerically integrated the resultant dynamic equations, and (b) event-driven (ED) simulations where the interaction potential between the particles is considered to be zero if they do not touch and infinite otherwise. Each has its own pros and cons.

The reasons to study granular matter is explained in the following section. The use of simulations introduced after it. Finally, the structure of the rest of the thesis is presented.

\subsection{Granular Matter}

Why is it important to study granular matter? They are the second most handled materials in the world after water. It behaves differently from any of the other forms of matter - solid, liquid or gases - and therefore should be considered an additional state of matter in its own right. See, for example, Refs. $[2,3]$.

Particulate systems of all scales are at the heart of a wide range of natural phenomena. From the vibrations of atoms within a fluid to the swirlings of interstellar dust, the behaviour of these many-body systems is a rich field for study.

Granular media, as an special case of particulate systems, appears in our daily life everywhere: the sand at the beach where we walk; the pharmaceutical pills we take; the cobble stones used for constructing the streets of our cities and buildings. The behavior of granular media is very rich, and it will depend on its packing density, pressure and forcing, whether it behaves like a solid, a liquid or a gas. For example, granular media with high densities are encountered in industrial sintering where they form extremely rigid solids. In other processes, the knowledge about the flow behavior of a more dilute granular medium such as suspensions or pastes is important.

In recent times the modelling of these systems has progressed rapidly thanks to large increases in computational power; however, our understanding of these systems and how the complex behaviour they exhibit arises is still quite limited.

There are two particularly important aspects that contribute to the unique properties of granular materials: ordinary temperature plays no role, and the interactions between grains are dissipative because of contact friction and the inelasticity of the particles. We might at first be tempted to view any gran- 
ular flow as that of a dense gas since gases, too, consist of discrete particles with negligible cohesive forces between them. In contrast to ordinary gases, however, the energy scale $k_{B} T$ is insignificant here. The relevant energy scale is the potential energy $P=m g d$ of a grain of mass, $m$, raised by its own diameter, $d$, in under the Earth's gravity, $g$. For typical sand, this energy is at least $10^{12}$ times the thermal energy at room temperature. Since $k_{B} T$ is irrelevant, ordinary thermodynamic arguments become useless.

In their now classical review, Jaeger, Nagel and Beringer [3] begin quoting the following line from Victor Hugo:

"Who could ever calculate the path of a molecule? How do we know that the creations of worlds are not determined by falling grains of sand?" (Victor Hugo, Les Miserables).

They aimed to show that Hugo's metaphor could have a literal meaning: "what happens to a pile of sand in a table is relevant to processes taking place on an astrophysical scale" [3].

Indeed, consider granular matter with long range interaction. The formation of planets is only due to two mechanisms: gravitational attraction and dissipative interactions amongst the dust particles. This can be easily simulated in a computer. Furthermore, thanks to today's computers and advanced algorithms, we can study astronomical processes literally on top of our tables without the need of a super-computer.

That is what we (mostly) did during this thesis: simulation of granular matter with advanced algorithms. In particular, we focused in granular gases and dense flows, each explained in what follows.

\subsubsection{Granular Gases}

Most of this thesis deals with structure formation on granular gases. By a granular gas we understand an ensemble of particles in a given spatial domain, be it two or three-dimensional, in the absence of gravity and that interact via dissipative collisions and/or long range potentials.

In contrast to classical gases, the dissipative character of the interactions makes that without energy input, the system is never stable. For an initially homogeneous system, as it dissipates energy, density fluctuation can give rise to the formation of large-scale structures and shear banding. Since there are not external forcing, granular gases are a perfect system of study where to understand the particularities of granular media.

We have studied how different theories predict this behaviour; a toy model for the formation of clusters and the consequent structures obtained with it; and the interplay between dissipation and long-range interaction and how this affects the structure formation. The guiding thread was always how 
to understand and model the ephemeral structures that born and die in a free-cooling granular gas.

\subsubsection{Granular Flow in Rotating Tumblers}

Rotating tumblers are widely used in industry, in processes as diverse as mixing, milling and calcination - the three processes present in the fabrication of cement, for example. They are one of the easiest experiments to perform with granular matter and present a rich phenomenology. Due to the movement of the wall, the grains flow when the angle of inclination is grater than the repose angle. Depending on the rotational speed, different flow regimes can appear: among others, sliding, continuous flow, centrifuging [4]. In the continuous regime, grains on the surface flow like in an avalanche while the bottom of the drum moves together with the walls.

When the material is not homogeneous, say composed of different size particles, the particles will segregate and form beautiful spatial patters [5]; fig. 1.1 shows one example of this. These patterns appear in the radial direction [6] - where the small grains tend to concentrate in the center of the drum - as well as in the axial direction [7] - where there is banding, i.e. the system separates in bands of mostly large and small particles along the axial direction - when the tumbler is long enough. In this thesis, we focus on the influence of container shape on the axial segregation.

\subsection{Simulations}

Nowadays, computer simulations have become a standard of scientific practice. For granular materials, the use of computer simulations has since long helped to study where the experiments cannot go and there is a standard set of methods for its study [8].

There are mainly two methods for the simulation of granular matter. The first consist in assuming perfectly rigid spheres that interact via instantaneous collision, realising the same assumptions of kinetic theory. This method is know as event-driven since the simulation advances through collisions, called events.

On the other hand, one can model the grains as soft particles that interact via a given interaction force.

Most models used in granular matter simulations are based on continuous potentials. However, discrete potentials exhibit much of the complex behaviour of real systems and provide some of the most elegant minimal models which exhibit complex behaviour.

In this thesis, we use both approaches. We use the event-driven method 


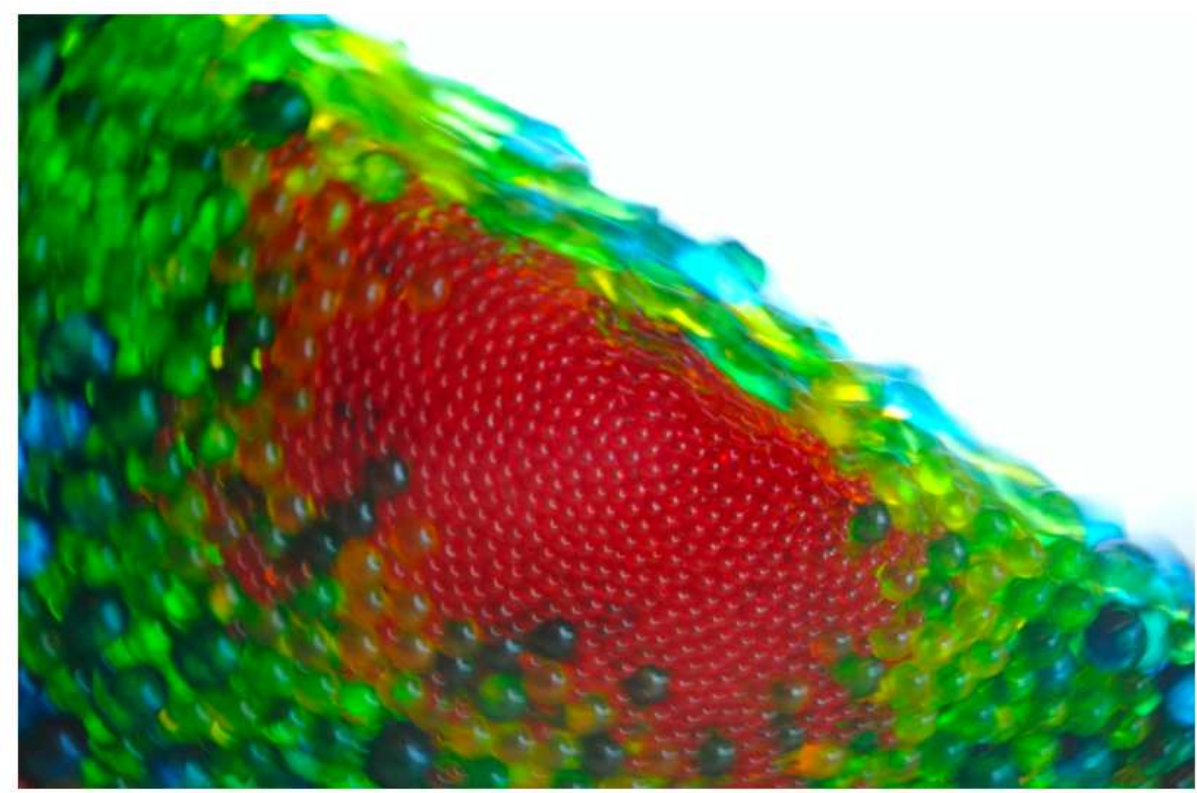

Figure 1.1: Picture of the avalanche in the centre of a rotating drum. Particles have different colour depending on their sizes: red, small; blue, large; yellow and green, medium sizes. The particles in the free surface flow in an avalanche while the particles in the bulk move solidarily with the drum (clockwise). For a detailed explanation of the experimental set up, see Chapter 4 .

to study the role of long-range interaction in the formation of clusters for free-cooling gases. For the simulation of granular flow in rotating tumbler, we the discrete particle method (DPM). In what follows, we introduce both models and the codes we have used in this thesis.

\subsubsection{Event-Driven Molecular Dynamics}

Discrete potentials have also played a major role in the development of molecular dynamics simulation methods. The very first molecular dynamics simulations were performed using discrete potentials because of their relatively inexpensive computational cost. Recent advances in the simulation of discrete potential systems have allowed the construction of molecular dynamics algorithms that scale linearly with the system size, allowing access to large system sizes and the long time scales required to extract the long time behaviour. Discrete potentials can even accurately approximate soft potentials, with possible savings in computational cost and time.

The simplest discrete potential is the hard-spheres model: the interaction energy is zero if particles are not in contact, and infinite if they are, hence creating instantaneous and binary collisions. A collision is called an event since something happens, and the state of the particles changes discontinu- 
ously.

By event-driven we mean that the state of the system is evolved in time from one event to the next. Since the dynamics can be solved analytically, the integration of the equations of motion is processed as a sequence of events rather than by fixed, small time-steps. After each event, the time of the next event in the system is calculated and the system advances in time. In brief, the algorithm consists of:

1. Given the instantaneous positions and velocities of all particles in the system,

2. predict the time of the next collision,

3. advance the time of the system to that instant, and

4. update the velocities of the particles that collide with a given collision rule; repeat from 1 .

For the details of the algorithm we refer the reader to Chapter 2 together with standard papers and books, see e.g. Refs. $[9,10]$.

In this thesis, we used two different codes. For Chapters 2 and 3, a code based on $[11,12]$ was used. For the results reported on Chapter 4, a new open source code was used instead: DynamoMD [13]. The advantage of using an open-source code is that it enables more collaboration with other scientist, since there is an standard to exchange works. Furthermore, DynamoMD includes many of the most recent advances in event-driven simulations and it is in continuous development.

\subsubsection{Discrete Element Method}

In contrast to what we did with the ED method, for the simulations with did not develop new methods but used existing algorithms. We used the code Mercury $^{1}$. Mercury is a code for discrete particle simulations. That is, it simulates the motion of particles by applying forces and torques that stem either from external body forces, (e.g. gravity) or from particle interaction laws (e.g. Lennard-Jones). For details see Ref. [14].

Particles are modeled as spherical and soft, so particles can overlap. Depending on the material model of the particles, the repulsion force can be obtained. In general, a spring-dashpot model is used, where there is a repulsive force proportional to the overlap, and a dissipative force proportional to the relative velocity. In this code, different contacts law are implemented [15]. For a given contact law, the resulting Newton's equations are integrated, and the temporal evolution of the system obtained.

\footnotetext{
${ }^{1}$ available at http://www2.msm.ctw.utwente.nl/athornton/MD/
} 
The biggest advantage of the Mercury code, besides it ease of use, is the inclusion of the Hierarchical Grid algorithm for contact detection [16]. With this algorithm, the simulation of arbitrary poly-dispersed system scales linearly with the number of particles.

\subsection{Thesis Structure}

In the second chapter we study the consequences of using more complex pair correlation functions at contact on the stability of an homogeneous 2D granular gas. Here we show that changing the pair correlation function at contact gives a better prediction for the cooling of dense systems and that new hydrodynamic modes are predicted at the transition density $(\nu \sim 0.7)$.

In the third chapter we develop a new even-driven cluster-cluster aggregation algorithm and study the evolution of the clusters formation for different densities. In our model, particles stick once they collide and do not rotate. We show that the fractal dimension of the final clusters depends on the initial density of the system.

Chapter four deals with the cooling of hard spheres with long range interaction either attractive or repulsive. We study the stability analysis for a modified cooling rate based on previous research, and realize ED simulations of the system, showing that in the dilute limit they fit perfectly the theory for the repulsive regime. In the attractive regime we obtain something similar to the cluster-cluster aggregation process we developed in chapter three, but this time taking into account the inelasticity of the particles. Due to the inelasticity, the structure formation is enhanced and a complex interplay between dissipation and attraction is found.

Chapter five deviates from the subject of granular gases and deals with the segregation of binary mixtures in a rotating tumbler. The rationale behind this research is the following: in our attempt to use advanced ED algorithms in the simulation of dense granular matter, we needed to develop a framework that would allow us to compare experiments, DPM and ED in a reliable way. The rotating drum was the obvious set-up for its experimental simplicity. However, since doing research means sometimes forgetting about your goals and listen to that the phenomenon has to say, we ended up by studying the segregation of binary mixtures in a concave rotating tumbler. We show how to control the axial segregation by modifying the shape of the tumbler. The results of this and the explanation of the phenomena is presented in this chapter.

In chapter six is the text I wrote for a talk I was invited to give in the music festival "Green Vibrations", in spring 2012 in Enschede. In this text, I give a personal account of what science represents for me and present a 
philosophical discussion concerning the way science is treated nowadays (by policy makers, scientific journals and scientists themselves). I added it to my thesis since I put the same intellectual effort I use in doing science in trying to understand how and why science is done in such a way today.

Finally, the conclusions and outlook close this work in chapter seven. 


\section{Chapter 2}

\section{Cooling of dense 2D hard-spheres gases*}

We show the differences in the stability properties of the Homogeneous Cooling State (HCS) of a two-dimensional mono-disperse collection of rigid and near-elastic disks, obtained by using different formulae for the pair-correlation function.

For an equation of state that takes into account the crystallisation and ordering of the particles (and the respective pressure drop), the critical wavelength of the heat conduction mode is considerably modified in the transition zone, involving a bifurcation and an additional mode of instability. The theoretical predictions, using the improved equation of state, are confirmed by numerical simulations. Nevertheless, some open questions remain.

\section{$2.1 \quad$ Introduction}

Structure formation in a granular gas has attracted much attention during the last decades (see for example, Refs. [17-26]). Starting from a macroscopically homogeneous system, structures evolve and a dilute granular gas coexists with denser, possibly much denser and even solid, clusters - in nonequilibrium. However, the coexistence of a fluid-like granular gas with a solid-like packing also occurs in many other systems, like during avalanche flow on inclined planes or in vibrated containers, see Refs. [27, 28] and references therein.

In the absence of walls and external forces, the crucial phenomena in a freely cooling granular gas involve the fluctuations in density, velocity and temperature, which cause position-dependent energy loss [18]. In denser areas, due to strong local dissipation, pressure and energy decay rapidly and material moves from 'hot' to 'cold' regions, there leading to even stronger

\footnotetext{
*Based on S. González and S. Luding, Eur. Phys. J. Special Topics 179, 55-68 (2010).
} 
dissipation and thus causing the density instability with ever growing (dense) clusters.

The freely cooling granular gas will be introduced first, as an example of a case where dilute and dense granular media co-exist. Even though the need to treat walls is avoided by using periodic boundary conditions, and the initial state is macroscopically homogeneous, resembling a classical, elastic hard sphere gas, the system develops interesting dynamics and structure formation - only due to the dissipative interactions of the particles, see Fig. 2.1 in section 2.2. Hydrodynamic equations and constitutive relations are introduced in section 2.3 and a stability analysis of these equations is presented in section 2.4. This has been done several times in the literature (see for example Refs. $[18,19,29,30])$, but always for a given (Enskog) pair correlation function (at contact distance, $g(\nu ; r=d)$ ). Our contribution is to study the consequences of an empirical pair correlation function on the instabilities of the HCS. Section 4.8 provides concluding comments.

\subsection{From homogeneous to inhomogeneous cool- ing}

When a homogeneous granular gas cools down due to collisional dissipation, one observes an initial homogeneous cooling state. Previous studies have shown that granular cooling results in the formation of structures: both mass and momentum density spontaneously become nonuniform. Four different regimes - kinetic, shearing, clustered, and collapsed - have been identified, (see Ref. [20] and references therein). For the clustered regime (the one we are interested in here), two stages with different energy dissipation behaviour can be identified: a cluster growth regime that follows the homogeneous state, and a final (inhomogeneous, non-equilibrium) state, where the cluster size is comparable to the system size and the structures span the whole system [18-20, 22,31-34].

Using the hard sphere model and event driven simulations, see e.g. Ref. [9], it is straightforward to simulate the time-evolution of a homogeneous granular gas with density (area fraction) $\nu=0.25$, about $N=2000$ parti-

cles of diameter $\hat{d}$, mass $\hat{m}$, and moderate dissipation with a coefficient of restitution $r=0.9$. 


\subsubsection{Collision Model}

For two particles, $p=1,2$, at positions $\vec{r}_{1,2}$, conservation of momentum leads to a collision rule:

$$
\vec{v}_{1,2}^{\prime}=\vec{v}_{1,2} \mp \frac{1+r}{2}\left(\hat{\vec{k}} \cdot\left(\vec{v}_{1}-\vec{v}_{2}\right)\right) \hat{\vec{k}}
$$

where the prime indicates the velocities $\vec{v}$ after the collision, and $\hat{\vec{k}}=\left(\vec{r}_{2}-\right.$ $\left.\vec{r}_{1}\right) /\left|\vec{r}_{2}-\vec{r}_{1}\right|$ is the unit vector pointing along the line of centers, from particle 1 to particle 2 .

At collision, the normal component, $v_{n}=\hat{\vec{k}} \cdot\left(\vec{v}_{1}-\vec{v}_{2}\right)$, of the relative velocity, $\vec{v}_{1}-\vec{v}_{2}$, changes its sign and is reduced by a factor $1-r$, with the coefficient of restitution $r$. Therefore, the kinetic energy related to the normal component is reduced by the factor $\epsilon^{2}=\frac{1-r^{2}}{2} \simeq 1-r$ for $r \approx 1$. The elastic limit, $r=1$, implies no dissipation $(\epsilon=0)$, while $r<1$ implies $\epsilon>0$.

$\tau_{E}=2.3$

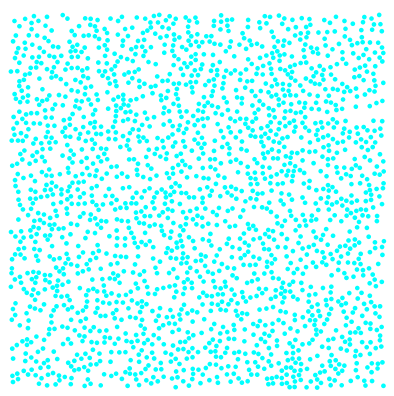

$$
\tau_{E}=2300
$$

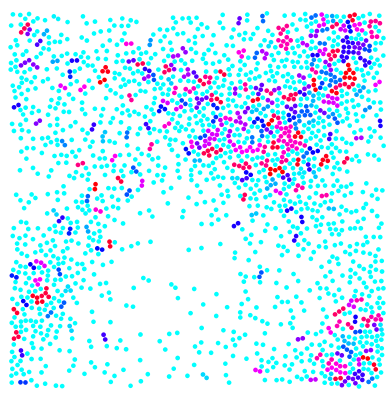

$\tau_{E}=23000$

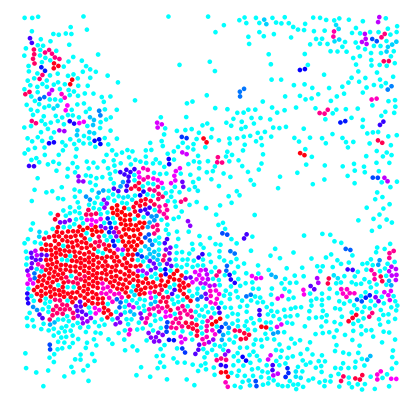

Figure 2.1: Two dimensional ED simulation snapshots at different dimensionless times $\tau_{E}$, for a periodic system with $N=2037$ particles, volume fraction $\nu=0.25$, and a coefficient of restitution $r=0.9$. The colour indicates if the particle is part of a cluster defined by distance, relative velocity, and angle of movement, as explained in the main text.

\subsubsection{Free Cooling Granular Gas}

Three snapshots at different (dimensionless) times, $\tau_{E}=\hat{t} / \hat{t}_{E}(0)$, with (dimensional) time $\hat{t}$ and initial collision rate $\hat{t}_{E}^{-1}(0)$, as defined in Eq. (2.11), are displayed in Fig. 2.1. Different colours (turquoise/light-gray for single particles) correspond to particles forming different "clusters". Clusters are defined as particles that are closer than $0.1 d$ and their relative angle of movement, given by $\arccos \left(\vec{v}_{1} \cdot \vec{v}_{2} /\left(\left|\vec{v}_{1}\right|\left|\vec{v}_{2}\right|\right)\right)$, is smaller than $30^{\circ}$. This definition is similar to the one used in Ref. [35], but differs from previous work [22,33] where only the relative position of the particles was considered. 


\section{$2.3 \quad$ Hydrodynamics}

Consider a mono-disperse system of particles of fixed mass whose collisions conserve momentum. The conservation of total mass and momentum is the (standard) basis for the macroscopic balance equations presented below. While mass and momentum are conserved, energy can be dissipated.

The constitutive relations needed to close the balance equations can be obtained on the basis of certain models and assumptions [3,21,22], part of which are presented below.

In this section, dimensional quantities are indicated by a hat.

\subsubsection{Mass balance}

Assume that $N$ particles with total mass, $\hat{M}=\sum_{p \in V} \hat{m}$, reside in a certain representative volume element (RVE), with volume $\hat{V}$. The continuity equation for the density $(\hat{\rho}=\hat{M} / \hat{V})$ reads:

$$
\frac{D \hat{\rho}}{D \hat{t}}+\hat{\rho} \frac{\partial \hat{u}_{i}}{\partial \hat{x}_{i}}=0
$$

with the average streaming velocity components, $\hat{u}_{i}=(1 / \hat{M}) \sum_{p \in V} \hat{m} \hat{v}_{i}^{p}$, the particle mass, $\hat{m}$, and the particle velocity components, $\hat{v}_{i}^{p}$. The substantial derivative (or material derivative) is defined as: $\frac{D}{D \hat{t}}=\frac{\partial}{\partial \hat{t}}+\hat{u}_{i} \frac{\partial}{\partial \hat{x}_{i}}$, and the sum over equal indices is implied.

\subsubsection{Momentum balance}

Momentum-conservation allows the momentum density $\hat{\rho} \hat{u}_{i}$ to change with time, not only due to a (momentum carrying) flux, $\hat{\rho} \hat{u}_{i} \hat{u}_{k}$, in or out of the RVE, but also due to inhomogeneous/directed forces (like during collisions) exerted from the outside on its interior, leading to:

$$
\hat{\rho} \frac{D \hat{u}_{i}}{D \hat{t}}=-\frac{\partial \hat{\sigma}_{i j}}{\partial \hat{x}_{j}}
$$

with the stress tensor components $\hat{\sigma}_{i j}$ on the right hand side. The stress can be split into an isotropic and a deviatoric part, $\hat{\sigma}_{i j}=\hat{p}^{I} 1_{i j}+\hat{\sigma}_{i j}^{D}$, with (isotropic) pressure, $\hat{p}^{I}$, where $1_{i j}$ denotes the unit tensor.

For a Newtonian fluid, the deviatoric stress is proportional to the rate of shear strain (symmetric, trace-free velocity gradient):

$$
\hat{\sigma}_{i j}^{D}=-2 \hat{\mu}\left(\frac{1}{2}\left[\frac{\partial \hat{u}_{i}}{\partial \hat{x}_{j}}+\frac{\partial \hat{u}_{j}}{\partial \hat{x}_{i}}\right]-\frac{1}{\mathcal{D}} \frac{\partial \hat{u}_{k}}{\partial \hat{x}_{k}} 1_{i j}\right),
$$


with the shear viscosity $\hat{\mu}$. Note that the isotropic pressure, $\hat{p}^{I}=\hat{p}+\hat{p}_{\zeta}$, also contains viscous terms, proportional to volume changes (divergence of the velocity field $\partial \hat{u}_{k} / \partial \hat{x}_{k}$ ), which are explicitly subtracted in Eq. (2.4), for $\mathcal{D}=2,3$ dimensions. The isotropic, strain rate dependent stress, $\hat{p}_{\zeta}=-\hat{\zeta} \frac{\partial \hat{u}_{k}}{\partial \hat{x}_{k}}$, contains the bulk viscosity, $\hat{\zeta}$. The pressure will be assumed to be dominated by its rate independent part, unless otherwise mentioned.

\subsubsection{Energy balance}

Energy-balance involves the kinetic energy density, $\hat{\rho} \hat{u}^{2} / 2$, due to the streaming velocity, $\hat{u}_{i}$, and $\hat{u}^{2}=\hat{u}_{i} \hat{u}_{i}$. In addition, there is also a fluctuating energy density, related to the fluctuating velocity $\hat{v}_{T}$ and to the "granular temperature",

$$
\hat{T}_{g}=\frac{2 \hat{E}_{\mathrm{kin}}}{\mathcal{D} N}=\frac{1}{\mathcal{D} N} \sum_{p \in V} \hat{m}^{p}\left(\hat{v}_{i}^{p}-\hat{u}_{i}\right)^{2}=\frac{\hat{m} \hat{v}_{T}^{2}}{\mathcal{D}},
$$

i.e., twice the fluctuating kinetic energy per particle per degree of freedom, where the sum runs over all $N$ particles in the averaging volume $V$. Note that in the following, we use $\hat{T}=\hat{T}_{g} / \hat{m}=\hat{v}_{T}^{2} / 2$. The energy-density balance then reads:

$$
\hat{\rho} \frac{D}{D \hat{t}} \hat{T}=-\hat{\sigma}_{i k} \frac{\partial \hat{u}_{i}}{\partial \hat{x}_{k}}-\frac{\partial \hat{q}_{k}}{\partial \hat{x}_{k}}-\hat{\gamma} \hat{T}
$$

with the energy density dissipation rate, $-\hat{\gamma} \hat{T}$. The right hand side of Eq. (2.5) contains also the rate of shear-heating, and the divergence of the heatflux. The latter contains the classical term proportional to the temperature gradient with the thermal conductivity, $\hat{\kappa}$, and a second, non-classical term, proportional to the density gradient with the corresponding transport coefficient $\hat{\lambda}[23,36]$, so that:

$$
\hat{q}_{k}=-\hat{\kappa} \frac{\partial \hat{T}}{\partial \hat{x}_{k}}-\hat{\lambda} \frac{\partial \hat{\rho}}{\partial \hat{x}_{k}} .
$$

Below we neglect the "non-classical" term since it is proportional to $1-r^{2}$ and we specialise to near-elastic systems, i.e, $r \sim 1$ so the non-classical contribution is of second order. The validity of this assumption has to be studied in future works.

\subsubsection{The ("classical") transport coefficients}

The equation of state (for the pressure), expressions for the shear and bulk viscosities and the heat conductivity, as well as the expression for the dissi- 
pation rate of the energy density, are collectively referred to as "expressions for the transport coefficients", for the sake of brevity. They are, a priori, not constant but dependent on the hydrodynamic fields and, possibly, also on their gradients or other terms, which are not considered and discussed here.

\section{Transport coefficients in 2D}

In $2 \mathrm{D}$, for a single species, in the elastic limit, $r \rightarrow 1$, in lowest order in powers of $1-r^{2}$ and the gradients, the transport coefficients can be expressed in various forms, see Ref. [37] for a detailed review.

All transport coefficients are proportional to $\hat{\rho}=\hat{\rho}^{p} \nu$, to powers of $\hat{v}_{T}$, and to powers of the product $s=s_{*} / \nu=(\nu g(\nu))^{-1}$, i.e., proportional to the non-dimensional mean free path, which depends on the pair-correlation function at contact $g(\nu)$. If one divides the transport coefficients by the combination $\hat{\rho} \hat{t}_{E}^{-1}$ that is common to all (see Luding's notation [37]), only powers of $\hat{d}, \hat{v}_{T}$ and $s$ remain as variables:

$$
\begin{aligned}
\hat{p} & =\hat{\rho} \hat{t}_{E}^{-1} \hat{v}_{T} \frac{\hat{d}}{2 \sqrt{2} \tilde{\gamma}}(s+2), \\
\hat{\mu} & =\hat{\rho} \hat{t}_{E}^{-1} \frac{\hat{d}^{2}}{4 \tilde{\gamma}^{2}}\left(s^{2}+2 s+\left(1+\frac{8}{\pi}\right)\right), \\
\hat{\zeta} & =\hat{\rho} \hat{t}_{E}^{-1} \frac{\hat{d}^{2}}{4} \\
\hat{\kappa} & =\hat{\rho} \hat{t}_{E}^{-1} \frac{\hat{d}^{2}}{\tilde{\gamma}^{2}}\left(s^{2}+3 s+\left(\frac{9}{4}+\frac{4}{\pi}\right)\right), \\
\hat{\gamma} \hat{T} & =\hat{\rho} \hat{t}_{E}^{-1} \epsilon^{2} \frac{\hat{v}_{T}^{2}}{2}
\end{aligned}
$$

with $\tilde{\gamma}=4 / \sqrt{\pi}$, and hiding the proportionality

$$
\hat{\rho} \hat{t}_{E}^{-1}=\sqrt{2} \tilde{\gamma} \frac{\hat{\rho} \hat{v}_{T}}{\hat{d} s} \propto \frac{\nu \hat{v}_{T}}{s}
$$

with the collision rate $\hat{t}_{E}^{-1}=2 \tilde{\gamma} \sqrt{\hat{T}} /(\hat{d} s)$. These equations have been obtained from a Chapman-Enskog expansion applied to the Enskog-Boltzmann equation in Ref. [38] and are frequently cited, e.g. in Refs. [26, 39-41].

In order to compare our results with the ones shown in Ref. [19], we normalise and non-dimensionalise the hydrodynamic equations. Lengths are scaled with particle diameter $\hat{d}$, masses by particles mass $\hat{m}$, and velocities with the initial thermal velocity $\sqrt{\hat{T}_{0}}=\hat{v}_{T}(0) / \sqrt{2}$. The hat that denotes the dimensional nature of the variable will be dropped after non- 
dimensionalisation, as for example:

$$
\hat{u}_{i}=\hat{T}_{0}^{1 / 2} u_{i}, \hat{T}=\hat{T}_{0} T, \hat{x}=\hat{d} x, \hat{t}=\hat{d} \hat{T}_{0}^{-1 / 2} t
$$

Consequently, the transport coefficients, in McNamara's notation [19], are defined as:

$$
\begin{aligned}
\hat{p} & =\tilde{p}(\nu)\left[\hat{\rho}^{p} \hat{d} \frac{\hat{T}}{\hat{s}}\right], \\
\hat{\mu} & =\tilde{\mu}(\nu) \nu^{-1}\left[\hat{\rho}^{p} \hat{d}^{2} \frac{\hat{T}^{1 / 2}}{\hat{s}}\right], \\
\hat{\zeta} & =\tilde{\zeta} \nu\left[\hat{\rho}^{p} \hat{d}^{2} \frac{\hat{T}^{1 / 2}}{\hat{s}}\right], \\
\hat{\kappa} & =\tilde{\kappa}(\nu) \nu^{-1}\left[\hat{\rho}^{p} \hat{d}^{2} \frac{\hat{T}^{1 / 2}}{\hat{s}}\right], \\
\hat{\gamma} \hat{T} & =2 \epsilon^{2} \tilde{\gamma} \nu\left[\hat{\rho}^{p} \frac{\hat{T}^{3 / 2}}{\hat{s}}\right],
\end{aligned}
$$

with $\hat{s} \equiv s \hat{d}^{*}$ The positive non-dimensional functions $\tilde{p}(\nu), \tilde{\mu}(\nu), \tilde{\zeta}, \tilde{\kappa}(\nu)$, and $\tilde{\gamma}$ are of order $\mathcal{O}(1)$ for any value of $\nu$. Their explicit form, as used in the next section, is given by:

$$
\begin{aligned}
\tilde{p}(\nu) & =s_{*}+2 \nu, \\
\tilde{\mu}(\nu) & =\frac{\sqrt{\pi}}{8}\left(s_{*}+\nu\right)^{2}+\frac{\nu^{2}}{\sqrt{\pi}}, \\
\tilde{\zeta} & =\frac{2}{\sqrt{\pi}}, \\
\tilde{\kappa}(\nu) & =\frac{\sqrt{\pi}}{2}\left(s_{*}+\frac{3 \nu}{2}\right)^{2}+\frac{2 \nu^{2}}{\sqrt{\pi}}, \\
\tilde{\gamma} & =\frac{4}{\sqrt{\pi}} .
\end{aligned}
$$

Note that the last term in the heat conductivity is different by a factor $2 \nu^{2}$ from McNamara [19], what we think is due to a typo in his paper, however, this term has a small effect only.

*We give McNamara's notation here since there is no standard in the literature, and it is useful to know how to translate form one to the other. 


\section{Pair-correlation functions}

Note that the pair correlation function $g(\nu)$ has not been specified as yet. In this chapter we will consider, as a working example, the differences between the predictions obtained by four of them: the classical (Enskog) function proposed by Henderson [42]:

$$
g_{2}(\nu)=\frac{1-7 \nu / 16}{(1-\nu)^{2}},
$$

the one proposed by Grossman et al. in Ref. [43],

$$
g_{\mathrm{pm}}(\nu)=1 /\left(\nu_{m}-\nu\right)
$$

where $\nu_{m}=\pi /(2 \sqrt{3})$ is the closest packing fraction in $2 \mathrm{D}$, and the high density limit of the previous one

$$
g_{\mathrm{fv}}(\nu)=g_{\mathrm{pm}}+(2 \nu)^{-1}
$$

which is evidently wrong at low densities since it diverges [37,44], and finally $g_{Q}(\nu)$, an empirical mix between $g_{2}$ and $g_{\mathrm{fv}}$, which takes into account the disorder-order phase transition at $\nu=0.7$, for which the explicit definition can be found in Ref. [37].

These functions are plotted in Fig. 2.2 to illustrate the differences. When compared with the non dimensional pressure obtained from ED simulations [44], the classical formula does not have the correct behaviour for high densities. In the range $\nu>0.75$, the high density limit formula $g_{\mathrm{fv}}$ behaves well, while $g_{\mathrm{pm}}$ behaves qualitatively well for all densities. The empirical $g_{Q}(\nu)$ performs quantitatively correct at all densities, including the disorder-order phase transition at $\nu_{c} \simeq 0.699$. In the inset of Fig. 2.2 we plot $P=2 \nu g(\nu)$, i.e., the non-dimensional collisional pressure. As can be seen from the data, when zooming in closer [44], the slope for $2 \nu g_{Q}(\nu)$ is always positive, whereas $g(\nu)$ has a negative slope in the transition zone.

In the rest of the chapter, we will assume that Eqs. (2.2), (2.3), and (2.5) are complete and sufficient to describe arbitrary flow conditions and rheology. This ansatz implies, that the flow behaviour of very dense, realistic granular matter can be already rather well described, in most cases, by using the constitutive relations in Eqs. (2.10) with an improved version of the paircorrelation function $g(\nu)$. In the following we will study how this works in the case of the stability analysis of the homogeneous cooling state. 


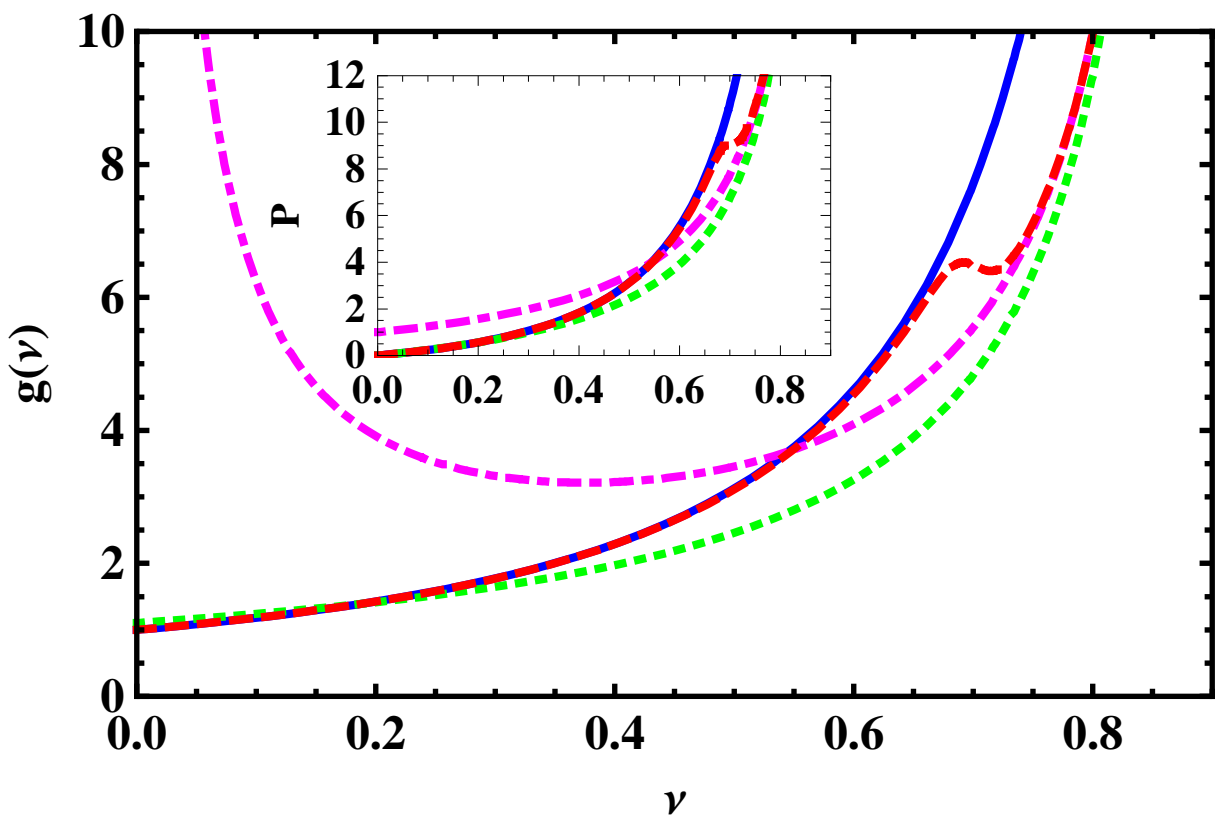

Figure 2.2: Pair-correlation function at contact. The solid blue line corresponds to $g_{2}$, the magenta dot-dashed line to $g_{\mathrm{fv}}$, the green dotted line to $g_{\mathrm{pm}}$ and the red dashed line to $g_{Q}$. The slope of the $g_{Q}$ curve around $\nu_{c}$ is strongly determined by the numerical coefficients, the width of the transition between low and high densities is $m_{c}=0.0111$, and the critical density is $\nu_{c}=0.699$, as in Ref. [37]. In the inset, the non-dimensional collisional pressure, $P=2 \nu g(\nu)$ is plotted against $\nu$. 


\subsection{Stability Analysis}

The homogeneous reference solution for a freely cooling gas is found by taking all the spatial derivatives in the hydrodynamic equations equal to zero, leaving just equation (2.5), non-dimensionalised as described above (and dropping density that occurs on both sides):

$$
\frac{d T}{d t}=-\frac{2 \tilde{\gamma} \epsilon^{2}}{s_{0}} T^{3 / 2}
$$

The solution is known as Haff's law [17], and can be written (following the notation of Ref. [19]):

$$
T(t)=\frac{1}{\left(\epsilon^{2} \tilde{\gamma} t / s_{0}+1\right)^{2}}
$$

with the other fields remaining homogeneous and constant:

$$
\nu=\nu_{0}, u:=u_{x}=0, v:=u_{y}=0
$$

The homogeneous-state variables and coefficients are designated by a subscript " 0 " $\left(\nu_{0}, p_{0} \equiv p\left(\nu_{0}\right), s_{0} \equiv s\left(\nu_{0}\right)\right.$, etc. $)$, and the derivatives with respect to density (evaluated at $\nu_{0}$ ) are denoted as, e.g., $p_{\nu_{0}} \equiv d p /\left.d \nu\right|_{\nu_{0}}$.

\subsubsection{Collision rate}

Starting from the initial configuration with homogeneous $\nu_{0}$ and $T_{0}$, the system dissipates energy at a rate that also depends on the energy of the system. As the energy of the system is dissipated, the relative velocities of the particles become smaller. The dynamics becomes slower, and with this, less energy is dissipated in each collision. The natural time unit of the system is thus the number of collisions per particle, given by the integral of the non-dimensional collision frequency $f(t):=T(t)^{1 / 2}=t_{E}^{-1}(t) \hat{d} s /\left(2 \tilde{\gamma} \hat{T}_{0}^{1 / 2}\right)$ :

$$
\tau \equiv \frac{1}{s_{0}} \int_{0}^{t} f\left(t^{\prime}\right) d t^{\prime}=\frac{1}{\epsilon^{2} \tilde{\gamma}} \log \left(1+\frac{\epsilon^{2} \tilde{\gamma} t}{s_{0}}\right)=-\frac{\log (f(t))}{\epsilon^{2} \tilde{\gamma}}
$$

\subsubsection{Linear equations}

In the following, we study the linear stability of the system in its new time variable, $\tau$, since then the equations can be written in the form $d \mathbf{Z}^{\prime} / d \tau=$ $F\left(\mathbf{Z}^{\prime}\right)$, with the vector of perturbed fields $\mathbf{Z}^{\prime}$. If we would keep the physical time as our variable, the equation for the temperature would be explicitly time-dependent. 
In order to linearise the hydrodynamic equations about the homogeneous but time-dependent solution (2.23), the fields are split into their homogeneous and their perturbation parts:

$$
\begin{aligned}
\nu & =\nu_{0}\left(1+\nu^{\prime}\right), \\
u & =f u^{\prime}, \\
v & =f v^{\prime}, \\
T & =f^{2}\left(1+T^{\prime}\right),
\end{aligned}
$$

where $\nu$ is the volume fraction, $u(v)$ the bulk velocity in $x(y)$ direction, and the primes indicate the perturbations. The primed quantities define $\mathbf{Z}^{\prime}$, and the factor $f$ is introduced for later convenience.

The final goal of this section is to examine the linear stability of small perturbations of the form $\exp (i \mathbf{k} \cdot \mathbf{x})$. Using Squire's theorem, that is, projecting the $2 D$ problem into one dimension, we will consider perturbations only along the $x$ direction so that the derivatives in the $y$ direction vanish. Taking the perturbations up to first order gives four coupled equations, by inserting the fields into Eqs. (2.2), (2.3) and (2.5):

$$
\begin{aligned}
\nu_{t}^{\prime} & =-f u_{x}^{\prime}, \\
\nu_{0}\left(f u^{\prime}\right)_{t} & =-p_{x}^{\prime}+\left(\mu_{0}+\zeta_{0}\right) f u_{x x}^{\prime}, \\
\nu_{0}\left(f v^{\prime}\right)_{t} & =\mu_{0} v_{x x}^{\prime} \\
\nu_{0}\left(f^{2} T^{\prime}\right)_{t} & =-p_{0} f u_{x}^{\prime}+\kappa_{0} f^{2} T_{x x}^{\prime}-\gamma_{0} f^{2} T^{\prime}-\gamma^{\prime} f^{2}-2 \nu_{0} f f_{t} \nu^{\prime},
\end{aligned}
$$

where the explicit expressions for the non-dimensional transport coefficients in the homogeneous state, $p_{0}, \mu_{0}, \zeta_{0}, \gamma_{0}$, and their perturbed values $p^{\prime}$ and $\gamma^{\prime}$ can be found in Ref. [19]

Changing to the new time variable $\tau$, using the identities $f=\exp \left(-\epsilon^{2} \tilde{\gamma} \tau\right)$, $d \tau / d t=f / s_{0}$, and scaling lengths by density as $X=\nu_{0} x$ and thus wave numbers as $K=\nu_{0}^{-1} k$, gives [19]:

$$
\begin{aligned}
\nu_{\tau}^{\prime} & =-s_{0}^{*} u_{X}^{\prime}, \\
u_{\tau}^{\prime} & =-\tilde{p}_{0}\left(T_{X}^{\prime}-s_{X}^{\prime}\right)-\tilde{p}_{\nu_{0}} \nu_{0} \nu_{X}^{\prime}+\left(\tilde{\mu}_{0}+\nu_{0}^{2} \tilde{\zeta}\right) u_{X X}^{\prime}+\epsilon^{2} \tilde{\gamma} u^{\prime}, \\
v_{\tau}^{\prime} & =\tilde{\mu}_{0} v_{X X}^{\prime}+\epsilon^{2} \tilde{\gamma} v^{\prime}, \\
T_{\tau}^{\prime} & =-\tilde{p}_{0} u_{X}^{\prime}+\tilde{\kappa}_{0} T_{X X}^{\prime}+2 \epsilon^{2} \tilde{\gamma} s^{\prime}-\epsilon^{2} \tilde{\gamma} T^{\prime} .
\end{aligned}
$$

Because the coefficients do not explicitly depend on time, we can perform the stability analysis for any choice of the pair correlation function $g(\nu)$, as long as it does not explicitly depends on temperature. 


\subsubsection{Shear instability}

The equation for the transverse velocity perturbation, $v^{\prime}$, that is the shear mode, is decoupled from the others. Assuming $v^{\prime}=\breve{v} \exp (\sigma \tau+i K X)$, with $\sigma$ the grow rate and the checked quantity, $\check{v}$, a constant, Eq. (2.35) reads:

$$
\sigma=-\tilde{\mu}_{0} K^{2}+\epsilon^{2} \tilde{\gamma}
$$

indicating growing shear instabilities for perturbations with wave numbers $K<K_{c}^{\text {shear }} \equiv \epsilon\left(\tilde{\gamma} / \tilde{\mu}_{0}\right)^{1 / 2}$.

In Fig. 2.3 we present the critical shear mode wave numbers (solid blue lines) as functions of the density for the four pair-correlation functions. Just the $g_{\mathrm{fv}}$ solution behaves differently (and incorrectly) for low densities, while the other three functions yield similar results since the dependence of $\tilde{\mu}_{0}$ on the pair-correlation function goes as $g(\nu)^{-2}$. In order to quantify the difference between the classical value and the one obtained with the modified pair correlation function, we plot the shear mode onset criterion normalised by the classical prediction (in Fig. 2.4 left). As expected, the only differences between $g_{2}$ and $g_{Q}$ occur for $\nu>0.65$, nevertheless, they are very small $(\sim 2 \%)$. In the case of $g_{\mathrm{pm}}$, for low densities it predicts an up to $10 \%$ bigger critical wavelength than $g_{2}$ (since $\left.g_{\mathrm{pm}}(\nu \sim 0)>g_{2}(\nu \sim 0)\right)$, while for intermediate densities it predicts an up to $5 \%$ smaller one. For high densities the prediction of $g_{\mathrm{pm}}, g_{\mathrm{fv}}$ and $g_{Q}$ are practically identical.

\subsubsection{Heat and sound instabilities}

Now, we must resolve three remaining coupled equations with five variables, which will give us the dispersion relation $\sigma=\sigma(K)$. Since $s=s(\nu)$ one has $s^{\prime}=\left.\frac{\nu_{0}^{2}}{s_{0}^{*}}\left(\frac{d s}{d \nu}\right)\right|_{\nu_{0}} \nu^{\prime}$, with this, and using the same plane wave ansatz as before, the equations read:

$$
\begin{aligned}
\sigma \check{\nu} & =-s_{0}^{*} i K \check{u} \\
\sigma \check{u} & =-\tilde{p}_{0}(i K \check{T}-i K \check{s})-i K \tilde{p}_{\nu_{0}} \nu_{0} \check{\nu}-K^{2}\left(\tilde{\mu}_{0}+\nu_{0}^{2} \tilde{\zeta}\right) \check{u}+\epsilon^{2} \tilde{\gamma} \check{u} \\
\sigma \check{T} & =-i K \tilde{p}_{0} \check{u}-K^{2} \tilde{\kappa}_{0} \check{T}+2 \epsilon^{2} \tilde{\gamma} \check{s}-\epsilon^{2} \tilde{\gamma} \check{T} .
\end{aligned}
$$

From Eq. (2.33) we have $\check{\nu}=-\frac{i K s_{0}^{*}}{\sigma} \check{u}$. Substituting the values of $\check{\nu}$ and $\check{s}$, and defining $a^{*} \equiv-\left.\nu_{0}^{2}\left(\frac{d s}{d \nu}\right)\right|_{\nu_{0}}$, we can write $\check{s}=i K a^{*} \check{u} / \sigma$, the dispersion relation is found to be: 

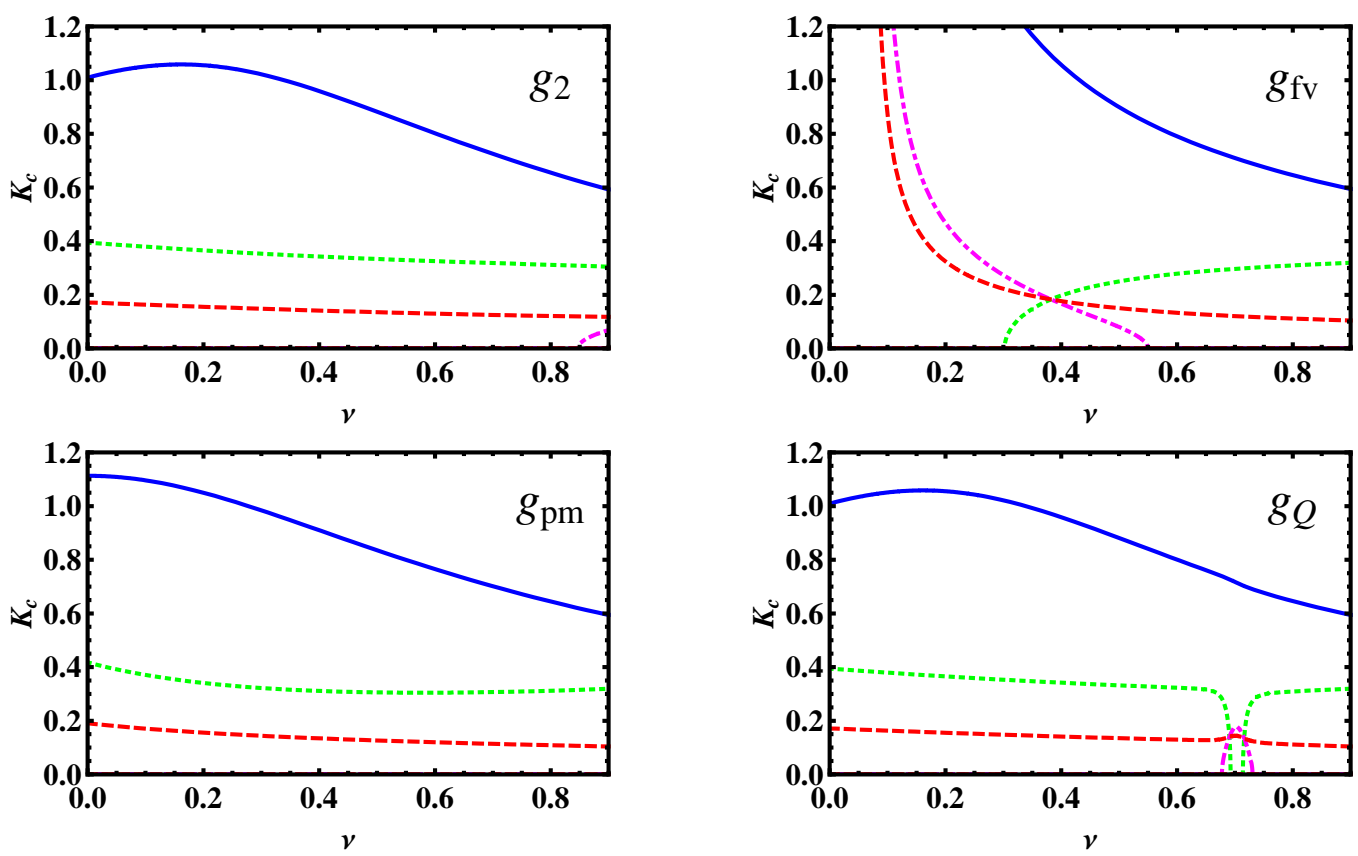

Figure 2.3: The critical wave numbers $K_{c}^{\text {shear }}$ (solid-blue) and $K_{c}^{\text {heat }}$ (dottedgreen), together with the other stability criteria (see [19]): $b>0$ (dashed-red) and $a b-c>0$ (dash-dotted-magenta). In the upper left figure we used $g_{2}$, in the upper right $g_{\mathrm{fv}}$, in the bottom left we used $g_{\mathrm{pm}}$, and in the bottom right $g_{Q} \cdot$

$$
\begin{array}{r}
\sigma\left(\sigma+K^{2} \tilde{p}_{0} \sigma a^{*}+\tilde{p}_{\nu_{0}} \nu_{0} K^{2} \frac{s_{0}^{*}}{\sigma}+K^{2}\left(\tilde{\mu}_{0}+\nu_{0}^{2} \tilde{\zeta}\right)-\epsilon^{2} \tilde{\gamma}\right) \\
\left(\sigma+\tilde{\kappa}_{0} K^{2}+\epsilon^{2} \tilde{\gamma}\right)+K^{2} \tilde{p}_{0}^{2} \sigma-2 \epsilon^{2} \tilde{\gamma} a^{*} K^{2} p_{0}=0
\end{array}
$$

This equation can be written in the form $\sigma^{3}+a \sigma^{2}+b \sigma+c=0$. It can be shown [19] that if any of $a, b, c$ or $a b-c$ are negative, then there exists a $\sigma$ with a positive real part, i.e., there exists an unstable mode. In this case, $a$ is always positive, but $b, c$ and $a b-c$ can become negative, indicating a growing instability.

The above criteria, together with the critical wavelength $K_{c}^{\text {shear }}$ are shown in Fig. 2.3 for different pair-correlation functions and a fixed coefficient of restitution $r=0.9$. Qualitatively $g_{2}, g_{\mathrm{pm}}$ and $g_{Q}$ have the same shape. Since $g_{\mathrm{fv}}$ is incorrect for low densities (and will not be further analyzed), the predictions are not valid and it even adds a new mechanism of instability for densities below $\nu \sim 0.4$. Since $g_{\mathrm{pm}}(\nu \sim 0) \neq g_{2}(\nu \sim 0)$, their critical wavelengths differ somewhat $(10 \%)$ for low densities.

Like for the shear mode, the predictions for the heat mode associated to $g_{2}$ and $g_{Q}$ are almost equal for low and moderate densities, differing around 

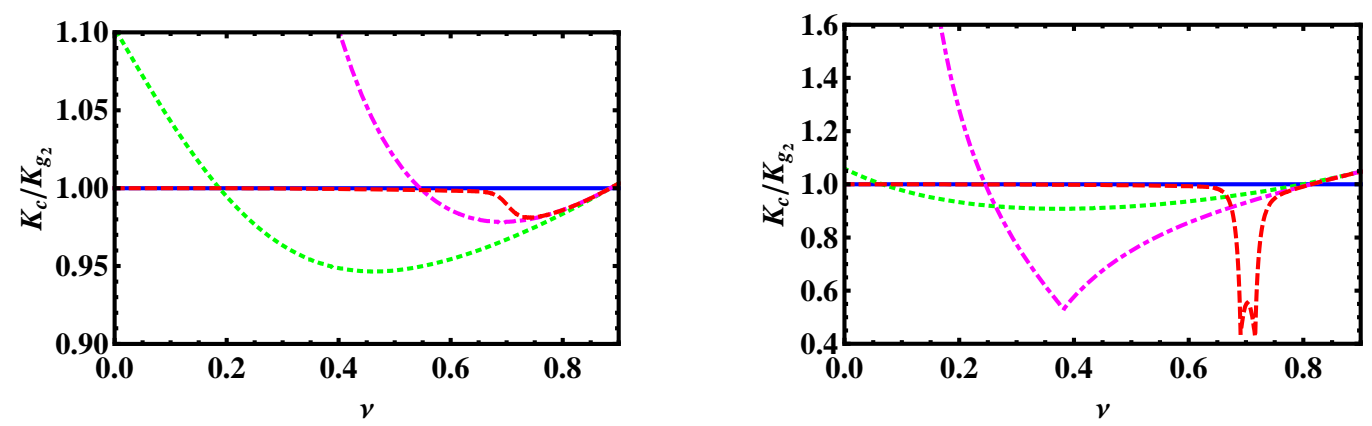

Figure 2.4: The critical wave numbers $K_{c}^{\text {shear }}$ (left) and $K_{c}^{\text {heat }}$ (right) for the four different pair-correlation functions normalised by the prediction given by the classical pair-correlation function $g_{2}$, and a fixed coefficient of restitution $r=0.9$. The solid blue line indicates identity and corresponds to $g_{2}$, the magenta dot-dashed line corresponds to $g_{\mathrm{fv}}$, the green dotted line corresponds to $g_{\mathrm{pm}}$ and the red dashed line corresponds to $g_{Q}$. For the shear mode the variations are of the order of $10 \%$ while for the heat mode the variations can be as big as $40 \%$ in the case of $g_{Q}$ (note the different scale in the vertical axis for both plots).

the critical density $\nu_{c} \approx 0.7$ and above. In the transition region, the theory based on $g_{Q}$ predicts a critical wavelength that is about $40 \%$ smaller than the classical value, and a new unstable mode appears.
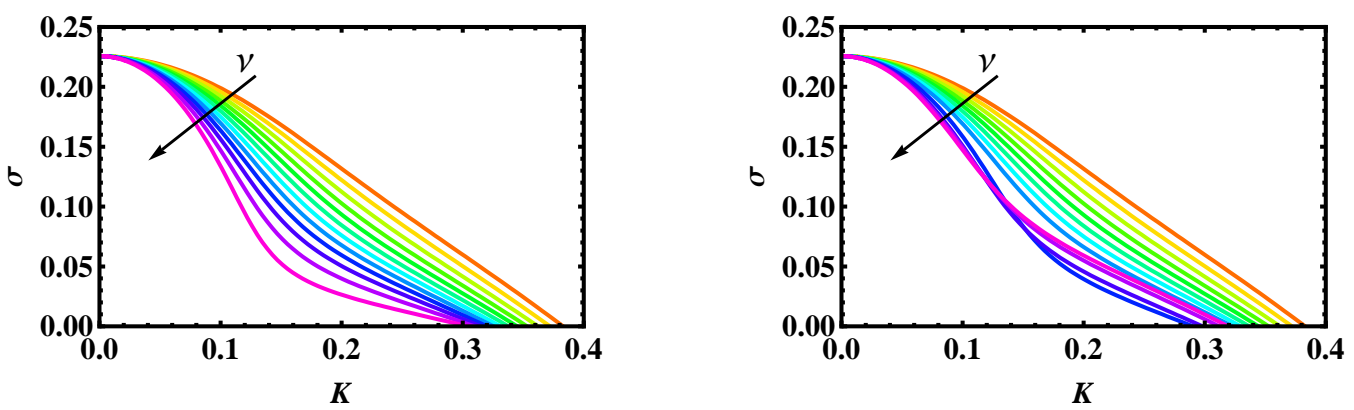

Figure 2.5: The dispersion relation for different $\nu$ increasing from $\nu=\Delta \nu=$ 0.075 up to $\nu=0.9$ in steps of $\Delta \nu$. In the upper left figure we used $g_{2}$, in the right $g_{Q}$, with a coefficient of restitution $r=0.9$.

In Fig. 2.5, we show the dispersion relation of the unstable modes, i.e. the positive roots of Eq. (2.40), for $g_{2}$ and $g_{Q}$ as a function of the scaled wave number $K=\nu_{0}^{-1} k$. The density is increasing from the top- to the bottomline, and the coefficient of restitution is again $r=0.9$. The dispersion relation for $g_{\mathrm{pm}}$ (away from $\nu=0.7$ ) is qualitatively similar to the one predicted by $g_{Q}$ and therefore is not shown.

For $\nu \geq 0.75$ the critical wave number $K_{c}$ (where the lines cross the horizontal axis) changes behaviour when $g_{Q}$ is used, i.e., the critical wave 
number is smaller (relative to the one predicted by $g_{2}$ ) at densities $0.675 \leq$ $\nu \leq 0.85$. Also, the shape of the dispersion relation is different. For the highest density, $\nu=0.9$, the critical $K_{c}$ is increased due to excluded volume effects, relative to the classical case with $g_{2}$.

For densities around $\nu=0.7$ a new mode appears for $g_{Q}$. As seen in Fig. 2.6 , for $\nu=0.7$ and $r=0.9$, the dispersion relation has two positive roots that merge around $K=0.115$. To illustrate the differences between the two new modes and the classical one, we plot the eigenmodes for $K=0.1$ in Fig. 2.7. The classical mode (Fig. 2.7 left) has $\sigma=0.158$ and is a temperature mode with small amplitude for velocity and density, which are shifted by $\pm \pi / 2$ respectively, from the temperature. The first new mode (Fig. 2.7 center) has $\sigma_{1}=0.135$, and is mainly a temperature mode, with small amplitude for velocity and a yet smaller amplitude for density, which are in phase and shifted by $-\pi / 2$ from the temperature. This mode is analogous to the classical mode (Fig. 2.7 left) but the velocity is shifted by $-\pi / 2$. The second new mode (Fig. 2.7 right) has $\sigma_{2}=0.020$ and the three fields have comparable amplitudes. The temperature is again the biggest mode, followed by the density and then the velocity. The phase shift is the same as in the previous mode, that is, velocity and density are shifted by $-\pi / 2$ from the temperature.
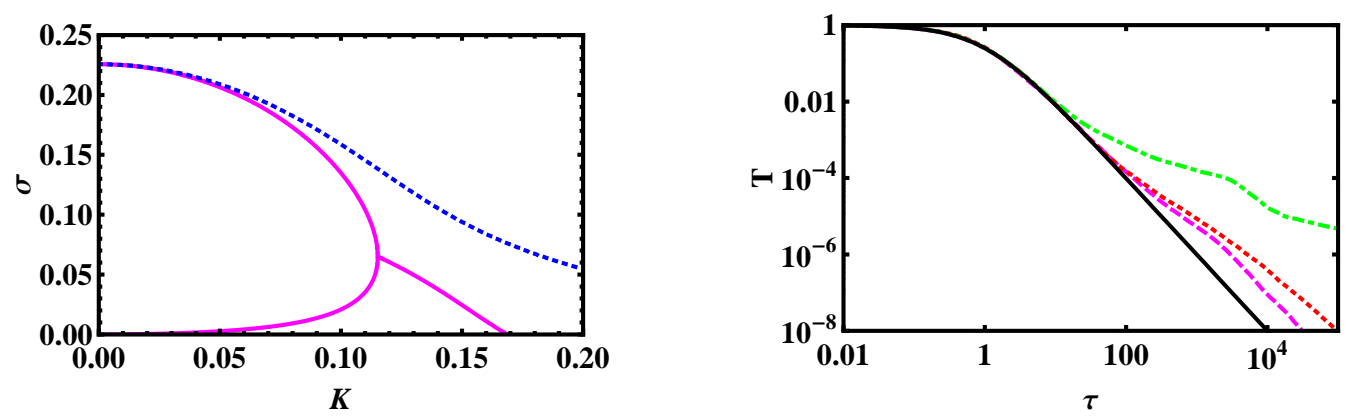

Figure 2.6: (Left) Dispersion relation for $\nu=0.7$ and $r=0.9$ for different pair-correlation functions. The magenta solid line is the one obtained by using $g_{Q}$, while the classical one is the dotted blue line. The onset of the instability is shifted to smaller $K$ when using $g_{Q}$. The bifurcation starts at $K_{b} \simeq 0.115$ and the new mode appears. (Right) The non-dimensional temperature as a function of the non-dimensional time, for $L=20$ magenta dashed line, $L=30$ red dotted line, and $L=100$ green dot-dashed line.

\subsubsection{Numerical Simulations}

To test the predictions of the theory, numerical simulations were carried out for three systems with density $\nu=0.7$ and $r=0.9$, for different sizes $L=\hat{L} / \hat{d}=20,30$ and 100 . The minimal wave numbers are $K_{m}=2 \pi \nu \hat{d} / \hat{\lambda}_{c} \simeq$ 

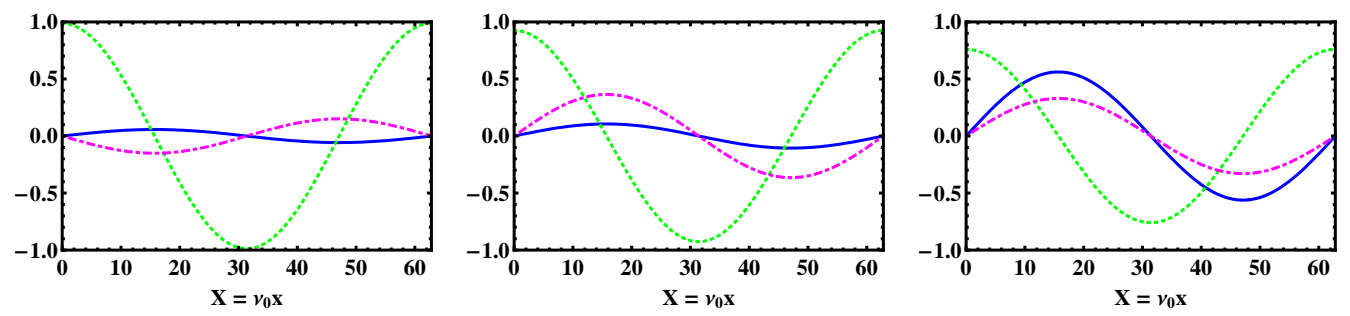

Figure 2.7: The unstable modes for $\nu=0.7, r=0.9, K=0.1$ using $g_{2}$ and $g_{Q}$. In the left panel, the modes for the classical pair-correlation function, in the middle panel the $\sigma_{1}$ mode (the fast one), and in the right panel the $\sigma_{2}$ mode (the slow one) for $g_{Q}$ are shown. The solid blue line is the density, $\nu$, the dotted green line is the temperature, $T$, and the dot-dashed magenta line is the velocity in the $x$ direction, $u$.

$0.20,0.14$ and 0.04 , respectively (see Fig. 2.6), where $\hat{\lambda}_{c}$ is the dimensional wavelength of the marginally stable wave, i.e., the smallest size of the system above which the instability is expected.

The system is thermalised in a way similar to [18]: starting from a square lattice with uniform random velocities, the system evolves during at least 100 collisions per particle with $r=1$, so that a Maxwellian velocity distribution is reached. A small $\left(\delta u \sim 0.1 v_{T}\right)$ sinusoidal perturbation of the velocity (in $x$ direction) is added to the initial state of the system and its evolution with $r=0.9$ is studied. The temperature evolution for the three systems is presented in Fig. 2.6 (right). After initial agreement, the systems deviate from Haff's law (solid line), the largest system first, the smallest last, as expected from the dispersion relation (see Fig. 2.6 left).

The dependence of Haff's law on the pair-correlation function is through $s_{0}$. In Fig. 2.6 we have used $g_{Q}$, which fits the data better than $g_{2}$. The ratio between the two pair-correlation functions for this density, $q_{g}=g_{2}(0.7) / g_{Q}(0.7)$ is equal to the inverse of the ratio of the $s_{0}$, that is $s_{0}^{g_{2}} / s_{0}^{g_{Q}}=0.84=1 / q_{g}$.

To quantify the role of the clustering instability, we measure the power spectra for the density fluctuations and the horizontal velocity, on the $x$ axis, averaged over the $y$ direction in bins of size $d$. In Fig. 2.8 we present the power spectra at three different times $\tau \simeq 0.001,5$ and 1000 for the $L=100$ system, for two different initial conditions, one perturbed as described above and the original thermal configuration. The power spectra are normalised so that the relative power of each mode is displayed. This way we can perform comparisons between different times, for which the amplitude of the velocity fluctuations is decreasing. The only system that displays a clear peak for low wave numbers, for both density and velocity fluctuations, is the one with $L=100$. The system with $L=30$ presents a qualitatively similar behaviour but slower, and the peaks are not as marked as for $L=100$. The $L=20$ 
system does not present a peak, showing that the clustering mode is not present and the deviation from Haff's law in this system is due to the shear mode instability.

In order to visualise the emergence of the clustering mode, a space-time diagram of the density fluctuations of the three systems is presented in Fig. 2.9. The system with $L=20$ does not develop a sinusoidal profile, at least for the time scales studied here. In contrast, for $L=30$, it is possible to identify a sinusoidal perturbation at $\tau \sim 10^{4}$, while the system with $L=100$ presents a clear sinusoidal behaviour already for $\tau \sim 10^{2}$, and later develops fluctuations with a larger wave number at $\tau \sim 10^{4}$.

The evolution of the smallest $K$, the one where the perturbation in the velocity is added, is presented in Fig. 2.10. The sinusoidal density mode that can be appreciated in Fig. 2.9, is reflected here in the growth of the relative power. For the system with $L=30$, a growth is observed at $\tau \sim 10^{2}-10^{4}$, while for $L=100$ the growth occurs earlier at $\tau \sim 0.1-10^{2}$. The smallest system does not present a clear growth of the field perturbation in the time scale studied.

To illustrate the change in density in real space, four snapshots of the perturbed system with $L=100$ are shown in Fig. 2.11. The first two snapshots from the left correspond to the same $\tau$ used in the right two plots of Fig. 2.8; the snapshot corresponding to $\tau=0.001$ is not shown due to its similarity with the one at $\tau=5$. The colour code represents the kinetic energy of each particle normalised by the average temperature at that instant. The system develops density fluctuations (clustering) that grow with time, to finally arrive to a sheared regime with two shear bands, visible in the right panel of Fig. 2.11.

\subsection{Conclusions}

In this chapter different constitutive models for the pair correlation function at contact have been used to predict the onset of instability in a freely cooling 2D granular gas. First, the classical $g_{2}$ proposed by Henderson [42], second the $g_{\mathrm{pm}}$ by Grossman et al. [43], third the high density limit of the latter, $g_{\mathrm{fv}}$ [44], and finally, the $g_{Q}$ proposed by Luding in Refs. [37,44].

Only the latter takes into account the disorder-order phase transition and the corresponding drop in the pressure around and above the critical density. This is also the one that results in the best fit with the data from ED simulations for mono-disperse disks.

While for low density the results do not depend on the choice of either $g_{2}$ or $g_{Q}$, the expected difference for the critical wave numbers at $\nu>0.75$ is rather small. The main result of this study is that around the crystallization 

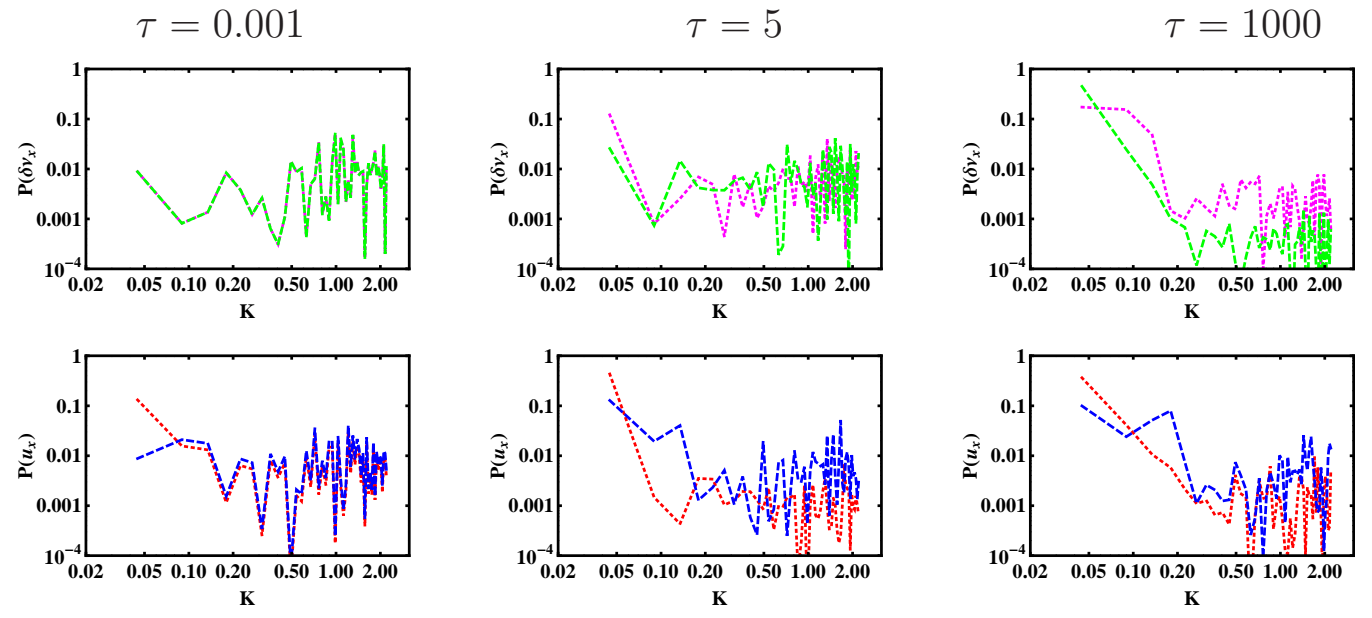

Figure 2.8: (Upper panel) Normalised power spectra of the density for three different times. The dotted magenta line corresponds to the perturbed system, and the dashed green line to the non-perturbed system. (Bottom panel) Normalised power spectra of the velocity in the $x$-direction, $u$, for the same three times as above. The red dotted line corresponds to the perturbed system, and the dashed blue line to the non-perturbed system. The contribution of the initial perturbation can be clearly seen in the first graph with the peak at $K \simeq 0.04$.

density $(\nu \simeq 0.7)$, the instability of the sound mode - that may precede the clustering instability - is delayed, and a new mechanism of instability appears. The growth rate of the new mechanism is one order of magnitude slower than the classical heat mode and it was not possible to excite it in the current simulations. Even though we present theory and simulations for moderate dissipation, the theoretical predictions of this study are strictly valid for smaller dissipation only. Whether it is justified to neglect the density gradient contribution to heat flux has to be studied elsewhere.

For a given dissipation and density $\nu=0.7$, using $g_{Q}$, theory predicts that in order to become instable under clustering, the system size must be approximately $40 \%$ bigger than previously predicted by using $g_{2}$. We have found that for $\nu=0.7$ the best fit for Haff's cooling law is given by $g_{Q}$, and that a system with $L=20$ is stable to clustering, in contrast to the theory using $g_{2}$. These are clear differences and should be checked by more systematically examining the onset of the clustering instability at these densities, by using hard sphere simulations. The present results support the use of a modified pair-correlation function even though open questions remain.

Furthermore, we observe that the inclusion of modified constitutive models does not change the mechanism of the instability for small dissipation: the shear and the heat conduction modes are the principally unstable modes. Nevertheless, in the critical region, for different pair-correlation functions, the 

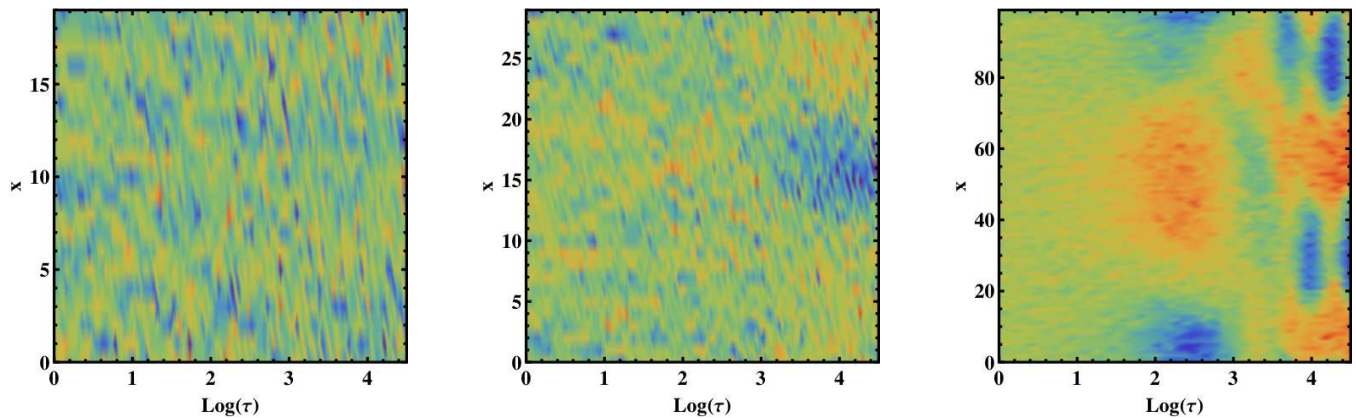

Figure 2.9: Space-time diagrams of the density fluctuations for the three perturbed systems, from left to right $L=20,30$ and 100 . The colour code shows in red the fluctuations above the average density, while in blue the fluctuations under the average density.
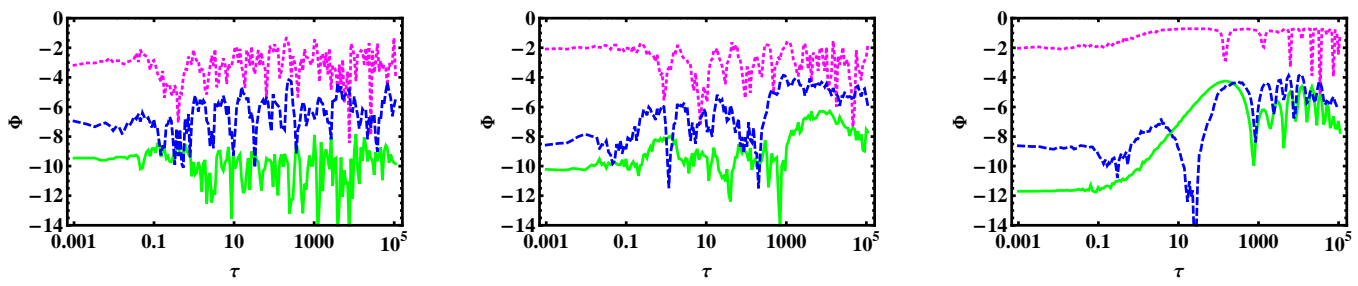

Figure 2.10: Time evolution for the first component of the normalised power spectra, $\Phi=\log \left(P\left(K_{m}\right)\right)$, in the three perturbed systems: from left to right $L=20,30$ and 100 . The magenta dotted line corresponds to the velocity, the dashed blue line to the temperature, and the solid green line to the density.

heat conduction mode is affected, while the shear mode is almost unchanged $(<10 \%)$. 

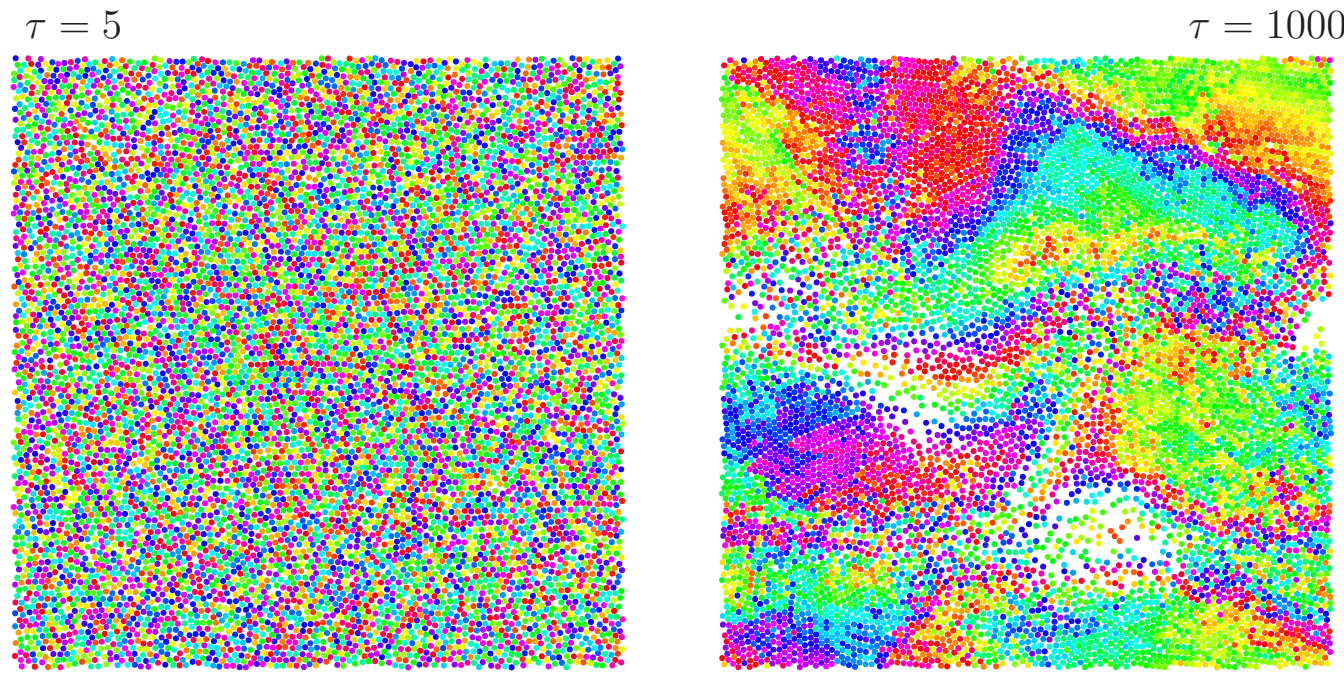

$\tau=10000$
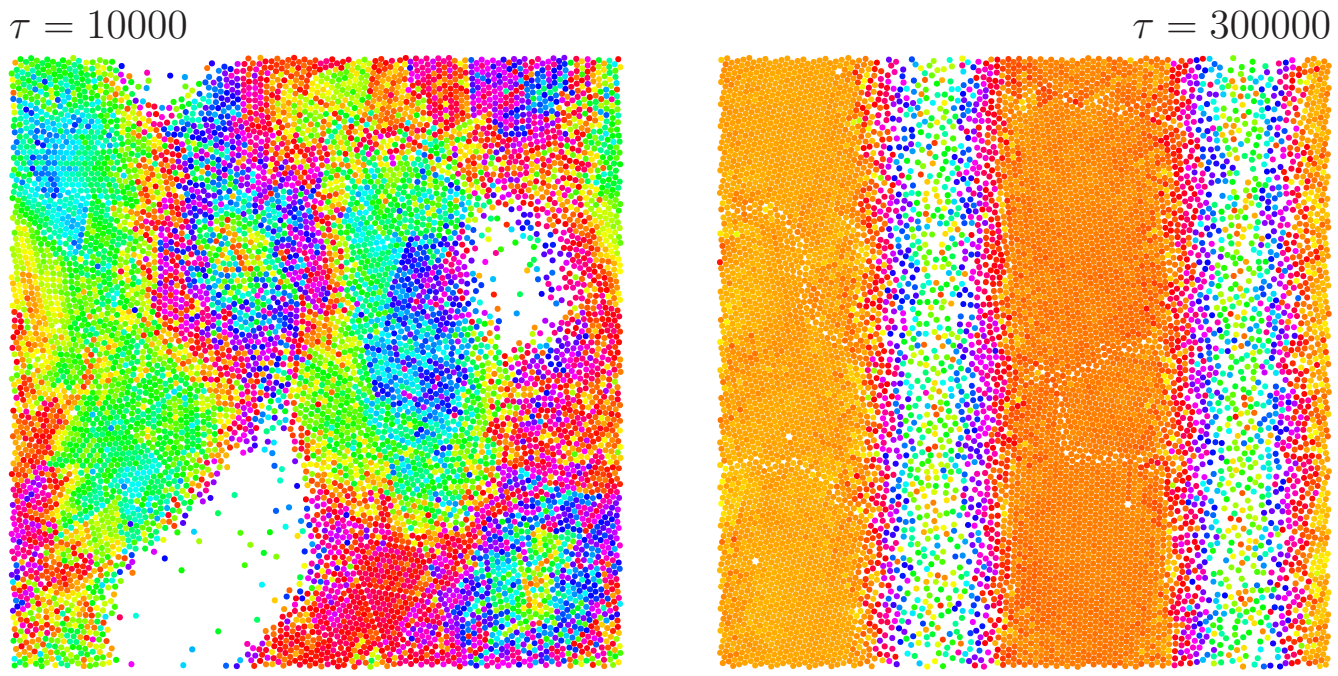

Figure 2.11: Four snapshots for the perturbed system with $L=100$ for times $\tau \simeq 5,1000,10000$ and 300000. The particles are colour coded depending on their relative kinetic energy normalised by the average. Red particles have more kinetic energy than the average, and blue particles have less. 


\section{Chapter 3}

\section{An Event-Driven Algorithm for Fractal Cluster Formation*}

A new cluster based event-driven algorithm is developed to simulate the formation of clusters in a two dimensional gas: particles move freely until they collide and "stick" together irreversibly. These clusters aggregate into bigger structures in an isotropic (random) way, forming fractal structures whose fractal dimension depends on the initial density of the system.

\subsection{Introduction}

Cluster formation is an important subject in various areas of physics; for example, in astronomy, ice clusters are believed to aggregate into planetesimals [45], the base of today's planets. In granular materials, the main theme of this chapter, tiny nano-Newton forces are responsible for macroscopic clusters in free falling jets [46]. These are similar to those that appear in nano jets from plasma physics [47]. Clusters are also found in granular avalanches [35], and air-driven granular beds [48].

Motivated by nano-aerosols [49], a cluster based event-driven algorithm is developed to simulate the formation of clusters in a $2 D$ gas with periodic boundary conditions: particles move freely until they collide and "stick" together irreversibly, moving as one cluster. The dynamics of the clusters is utterly simplified in our model. Conserving only linear momentum during collisions, angular momentum is disregarded. These clusters evolve and aggregate into bigger fractal structures, whose dimension $d_{f}$ is found to be in the range $1.4<d_{f}<2$; in contrast, to the case of diffusion-limited aggregation (DLA), where $d_{f}=1.67$ [50]. Here, we keep track of the dynamics of the clusters instead of adding particles one by one like in DLA. This procedure

*Based on S. González, A.R. Thornton, S. Luding, Computer Physics Communications, 182, 9, (2011). 
can be seen as a mix between irreversible coalescence [51], and a lattice-free version of a cluster-cluster aggregation model [52].

Implementing clusters in an event-driven algorithm has two advantages: Firstly, defining clusters of particles avoids the need to predict the events between particles of the same cluster. Since particles in a cluster move together as a rigid solid, they cannot collide. This alone decreases the computational effort required to simulate the clusters, where in standard event-driven models most of the collisions occur [33]. Secondly, the concept of clusters appears in a wide range of particulate physics: granular structures develop long correlations in space and time; see for example, Keys et al., Ref. [48], where it is found that particles move in one-dimensional paths ("strings") that aggregate into clusters.

In the next section we explain the algorithm used and how it is related to the classical event-driven model. After that, we present a selection of numerical simulations. Finally, concluding remarks and plans for future work are discussed.

\subsection{Algorithm}

By event-driven we mean that the state of the system is evolved in time from one event to the next. After each event, the time of the next event is calculated and the system advances. For the details of the algorithm we refer the reader to chapter 2 together with standard papers and books, see e.g. Refs. $[9,10]$. In brief, the algorithm consists of:

1. Given the instantaneous positions and velocities of all particles in the system,

2. predict the time of the next collision,

3. advance the time of the system to that instant, and

4. update the velocities of the particles that collide with a given collision rule, and repeat from 1.

The event-driven algorithm presented here builds on previous work, where the static phase in dense granular systems was simulated with a different dynamics, also improving the performance see Ref. [12] and the second chapter of this thesis. This is a necessary step towards a multiple-scale event-driven simulation for granular matter, where each cluster can have its own dynamics and collision rules.

The kind of clusters we are interested in at the moment are, e.g., suspensions of nano-particles in a gas, which stick together at contact due to Van 
der Waals forces (as in Ref. [53]). In reality, clusters of particles conserve angular momentum when they collide, which induces rotation in the clusters. For the sake of simplicity, and since (at the moment) we are mainly interested in the algorithm rather than in recovering the right physics, we will disregard rotations of the clusters and, hence, consider only translational motion. This approximation should be valid for fairly dilute systems and break down as the density of the system increases. Note that, anyway, no considerable rotation of large clusters is expected in a gas.

In normal event-driven algorithms one has to predict the next collision between all two-particle pairs. In this version, we introduce a new object called cluster (which may consist of just one particle or many), and only collisions between these objects have to be computed. Since a cluster consists of a finite number of particles, the position of a particle $i$ within a cluster $C$, is given by

$$
\vec{r}_{i, C}(t)=\Delta \vec{r}_{i}+\vec{r}_{0}+\vec{v}_{C} t
$$

where $\vec{v}_{C}$ is the linear velocity of the cluster and $\Delta \vec{r}_{i}$ relative position of the particle $i$ in the cluster $C$. The time is measured since its last collision, and $r_{0}$ is the centre of mass of the cluster at that instant.

Now that we have defined the evolution of particles within a cluster, collisions between particles in different clusters can be detected. This is a massive time saving as collision between particles within the same cluster do not have to be checked for, and as the size of the clusters increases the total number of checks decreases. Once the collision of two clusters has been carried out, the colliding particles "stick" together and the two clusters are combined into a single larger one. The velocity of the newly formed cluster is calculated by considering the conservation of linear momentum only. This process is repeated until the system consists only of a single cluster.

The classical event-driven model needs to deal with a quadratic equation, both in the case with or without gravity. Like in the classical case, here we have to find the time of collision between two particles $i, j$ by (analytically) finding the first (smallest) positive root of

$$
\left|\vec{r}_{i}(t)-\vec{r}_{j}(t)\right|^{2}=d^{2}
$$

with $d$ the diameter of a particle. The inclusion of rotating clusters in the simulation makes the equation to find the collision time highly nonlinear. Furthermore, the number of roots cannot be a priori determined making the problem extremely difficult. Recently, methods have been developed to deal with these situations $[54,55]$, but the inclusion of rotation is beyond the scope of this chapter.

Summarizing the simulation procedure, one has to: 
1. Start with an initial configuration of particles,

2. find the time for the next collision in the system.

3. Advance the system to that instant and merge the two particles (clusters) into a single cluster,

4. predict the next event with the new configuration, and

5. repeat until all the energy is dissipated and a single cluster is present in the simulation (the simulations are run in the centre of mass reference frame).

Three snapshots of a simulation are shown in Fig. 3.1. At the beginning of the simulation, (a) particles are arranged in a square lattice with random velocities (each component of the velocity is take from an uniform distribution with zero mean). The colour code represents different clusters in the simulation. At this initial time, every cluster correspond to strictly one single particle. At a later time, (b) clusters of different size coexist in the simulation and aggregate as soon as they are in contact. Finally, (c) the system contains only two clusters that will collide in the next event of the simulation, form one cluster, and end the aggregation process.

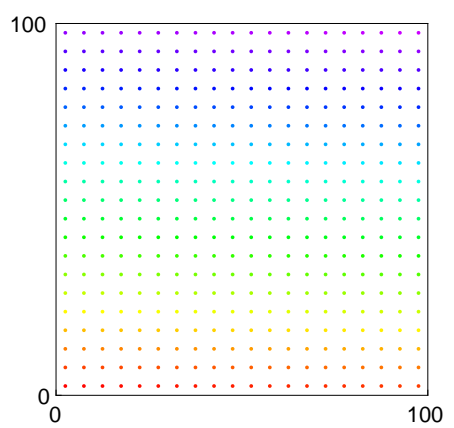

a

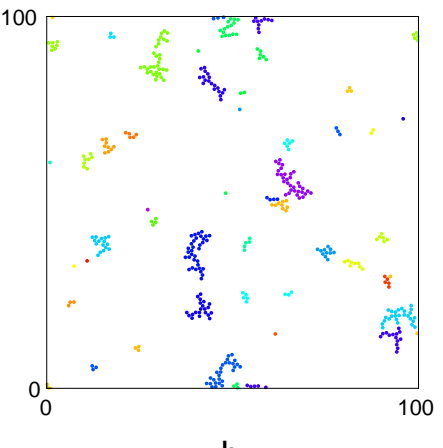

b

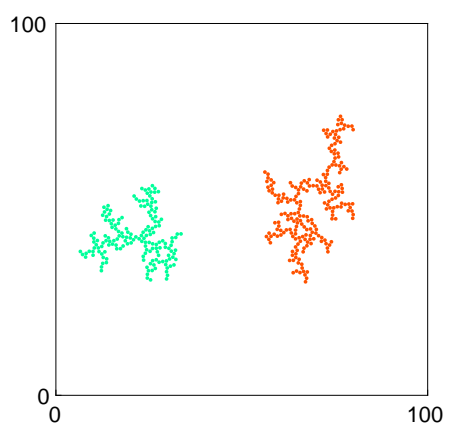

C

Figure 3.1: Three snapshots during the evolution of a system of $N=400$ particles in a box of size $L=100 d$, a packing fraction of $\nu \simeq 0.03$. Each colour represents a different cluster. Time increases from left to right.

\subsection{Simulations}

The simulation consists of a system of $N$ particles in a $2 D$ square box of size $L$ with periodic boundary conditions. Particles are mono-disperse with diameter $d$ and mass $m$. The packing fraction of the system is given by $\nu=N \pi d^{2} /\left(4 L^{2}\right)$. In order to start with a homogeneous configuration, we let 
the system equilibrate: starting from a square lattice, each particle collides at least 10 times elastically until a homogeneous regime is reached with average velocity $v_{0}$. Once thermalised, the clustering algorithm is switched on, and the simulation runs until one big cluster is formed.

\subsubsection{Temporal Evolution}

The natural time scale is the initial Boltzmann mean collision time (as defined in [51]), $\tau_{0}=\left(4 d \pi^{2} / v_{0}^{2}\right)^{1 / 2} / \nu$, with $\nu$ the packing fraction, and $g(\nu) \simeq 1$ for low densities.

The scaling behavior of the energy was studied. For dilute systems, the mean kinetic energy per particle follows a power law $\left\langle E_{K}\right\rangle /\left\langle E_{K}\right\rangle_{0} \propto \tau^{-\delta}$, with $\delta=1.3$ for almost four decades, as can be seen in figure 3.2. This results is similar to the coalescence model from Ref. [51], where particles move freely until they collide and merge into a single particle of larger mass and radius (instead of a cluster, as in our model) and a scaling with $\delta=1.12$ was found. This scaling breaks down when the number of clusters is small and finite size effects become important, leading to bad statistics, since we did not employ ensemble averaging.

For these systems, the average cluster size also follows a power law scaling $\left\langle S_{C}\right\rangle \propto \tau^{\zeta}$ with $\zeta=1$, which corresponds exactly to the mean field predictions in Ref. [56].

\subsubsection{Cluster size distribution}

As the simulation evolves, the distribution of clusters develops from $N$ clusters of size one (free particles), to one cluster of size $N$. The change of the cluster size distribution as a function of time is plotted in figure 3.3. Since the raw probability density function (PDF) is noisy, see Fig. 3.4, we plot the cumulative distribution function $(\mathrm{CDF})$ as a function of cluster size for different non-dimensional times. The data presented here correspond to $N=10^{6}$ and a fairly dilute packing fraction of $\nu=0.0097$. As time increases the number of clusters decreases and the distribution broadens, i.e. the difference between the biggest cluster and the smallest becomes larger, reaching a maximum around $\tau \sim 924$.

The resulting PDF cannot be fitted by an exponential function as in [51]. For intermediate times a fit of the form $P(s ; \tau)=a(\tau) s^{-\gamma(\tau)} \exp (-w(\tau) s)$, with $a(\tau), \gamma(\tau)$, and $w(\tau)$ are time dependent free parameters, while $s$ is the cluster size. This can reproduce the qualitative behavior of the distribution: Fig. 3.4 shows the PDF for three intermediate times together with the best fit. The numerical values for the coefficients appear in the caption of the figure. 


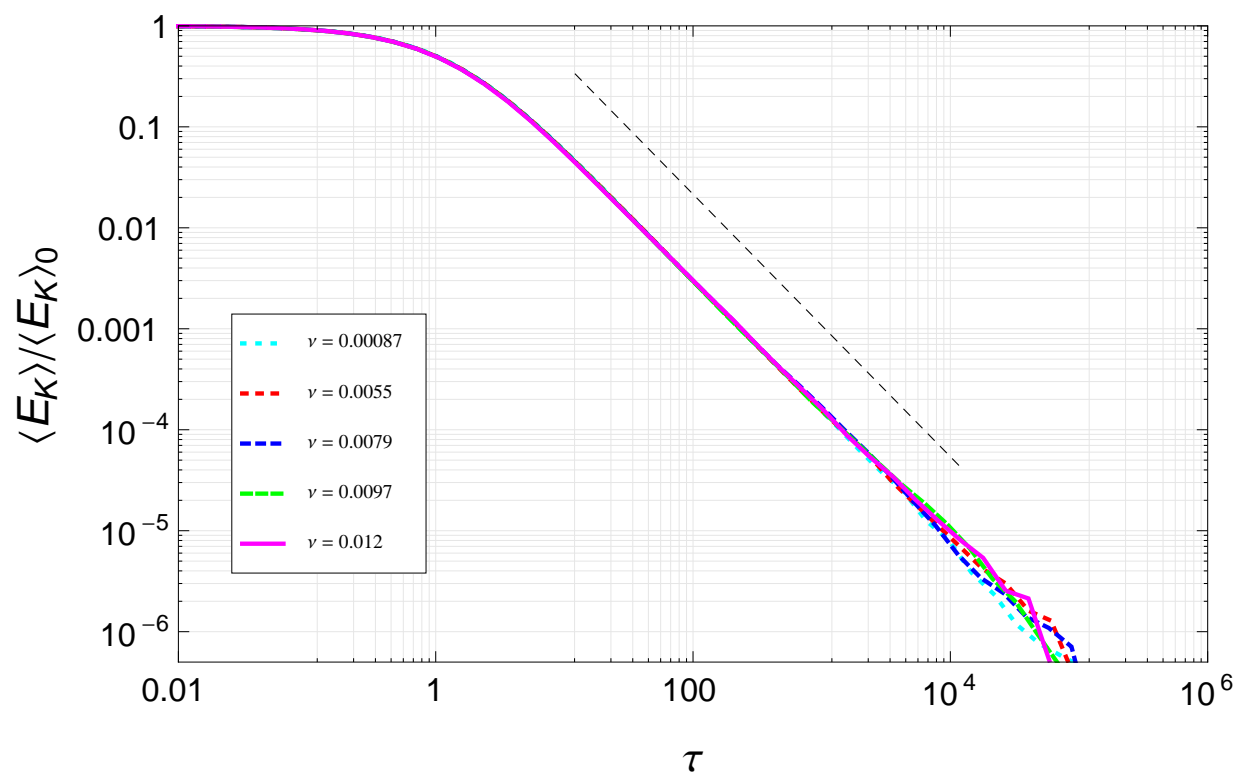

Figure 3.2: Energy as a function of the non-dimensional time $\tau=t / \tau_{0}$ for five systems with $N=10^{6}$ and packing fractions in the range $0.0008 \leq \nu \leq 0.012$. The energy follows a power law $\left\langle E_{K}\right\rangle /\left\langle E_{K}\right\rangle_{0} \propto \tau^{-\delta}$ with $\delta=1.3$, as indicated by the dashed line

\subsubsection{Fractal dimension and density}

With the final configuration from each simulation, we count the number of particles present in a circle of radius $r$ around ten randomly chosen particles of the cluster. We do this to obtain the number distribution $n(r)$, whose exponent is the fractal dimension of the system. We confirmed that the fractal dimension was almost independent of the points selected, by choosing points in the inner third, and in the outer third of the fractal: both measurements lead to practically the same results; here, we present data for $d_{f}$ based on inner points.

The fractal dimension we obtain is strongly dependent on the density of the system. If we start with a very dense system, there is no re-arrangement possible and the final state will practically coincide with the initial state. Due to this, an integer dimension of $d_{f}=2$ is expected for dense systems. For vanishing density, we expect an asymptotic lower fractal dimension, since after some point the mean free path is much larger than the cluster size, i.e., the system is so dilute that molecular chaos holds.

Figure 3.5 shows the fractal dimension plotted against the density for different systems. To measure the effect of the density, we vary the size of the system for a given number of particles $N=10^{6}$. The system sizes chosen are in the range $40000 d \geq L \geq 1000 d$, corresponding to densities between $0.0005 \leq \nu \leq 0.78$. We have realized one simulation for each system size, 


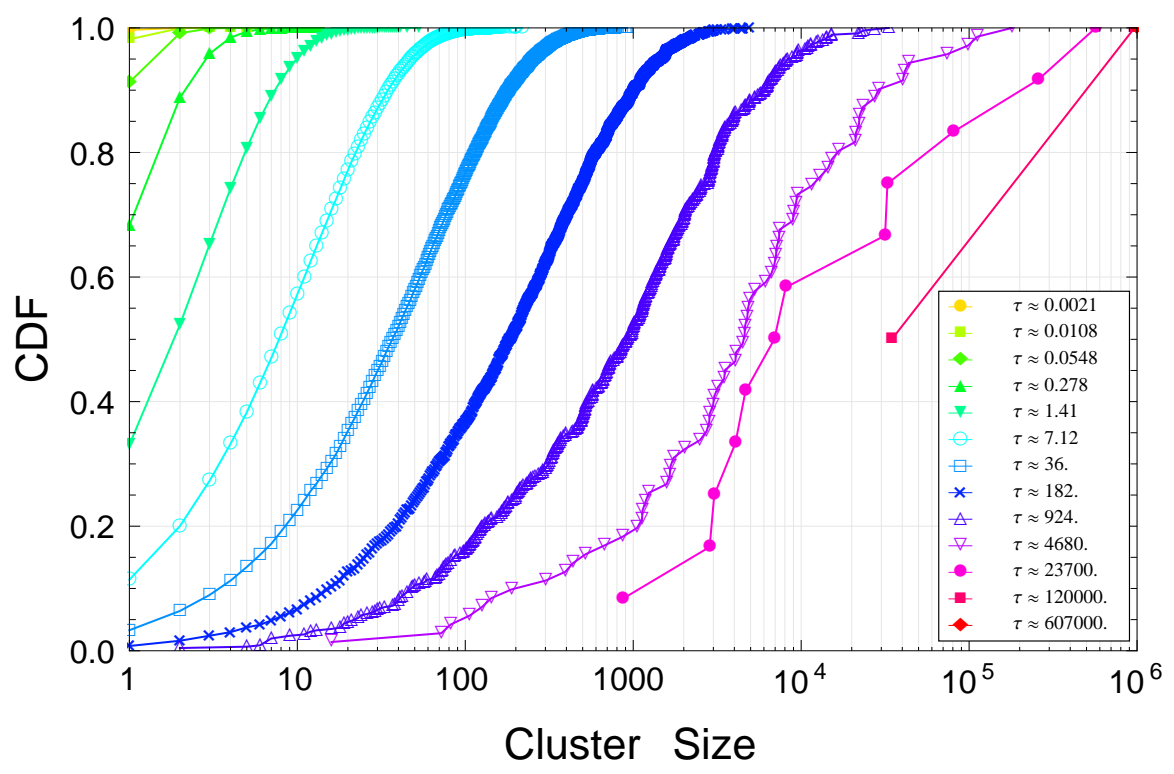

Figure 3.3: Cumulative distribution function for different non-dimensional times $\tau$ for a system of $N=10^{6}$ and $\nu=0.0097$. As time increases the number of cluster decreases and the distribution broadens, reaching a maximum around $\tau \sim 924$. Eventually, just two clusters are present: (red) squares. On the plot, each symbol (data point) represents one cluster, so we can see that as long as there are some free particles in the system, the distribution is smooth.

but gather statics by choosing different central particles.

The error bars correspond to the fluctuations in the measurement of the fractal dimension on a single simulation, and not to different realizations for the same system. As expected, for high densities the fractal dimension approaches 2 , namely for $d_{f}(0.78)=1.97 \pm 0.01$, that is, the cluster approaches a two dimensional structure. For vanishing densities it is found that $d_{f}(\nu \rightarrow 0)$ does not reach a clear asymptotic value and decreases with density, at least for the few values studied here. This fractal dimension is considerably smaller than the one found, by Witten et al. for the diffusionlimited aggregation process [50], where the fractal dimension is $d_{\mathrm{DLA}}=1.67$.

\subsection{Conclusions}

In this chapter we have presented event-driven simulations of irreversibly aggregating clusters in $2 D$ systems of various densities. These clusters have non-physical dynamics but represent a "toy" model that permits us to understand how to make cluster simulations in an event-driven algorithm. The formation of fractals was studied, and the exponent found depends strongly on 


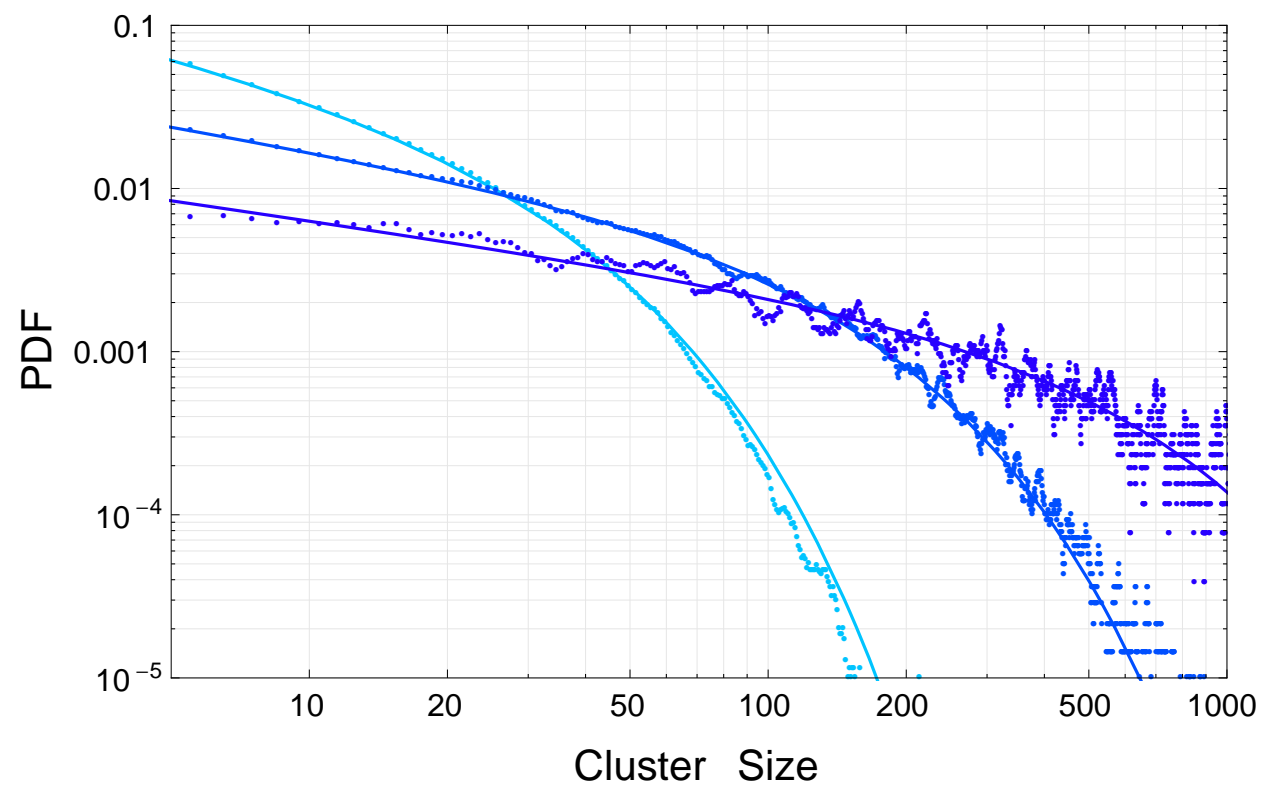

Figure 3.4: Probability distribution function for $\tau=7.12,36$, and 182 and the same system of Fig. 3.3. The distribution flattens and becomes more noisy as time passes. The solid lines correspond to the fit, while points correspond to the data. The functions plotted are $P(s ; 7.12)=0.2(1) s^{-0.63(1)} e^{-0.038(1) s} ; P(s ; 36)=0.052(1) s^{-0.46(1)} e^{-0.0086(1) s} ;$ and $P(s ; 182)=0.016(1) s^{-0.40(1)} e^{-0.0020(1) s}$ from top to bottom.

the initial density of the system, with dimensions in the range $1.4 \leq d_{f} \leq 2$. The denser the system, the closer to a two dimensional structure the fractal is. 


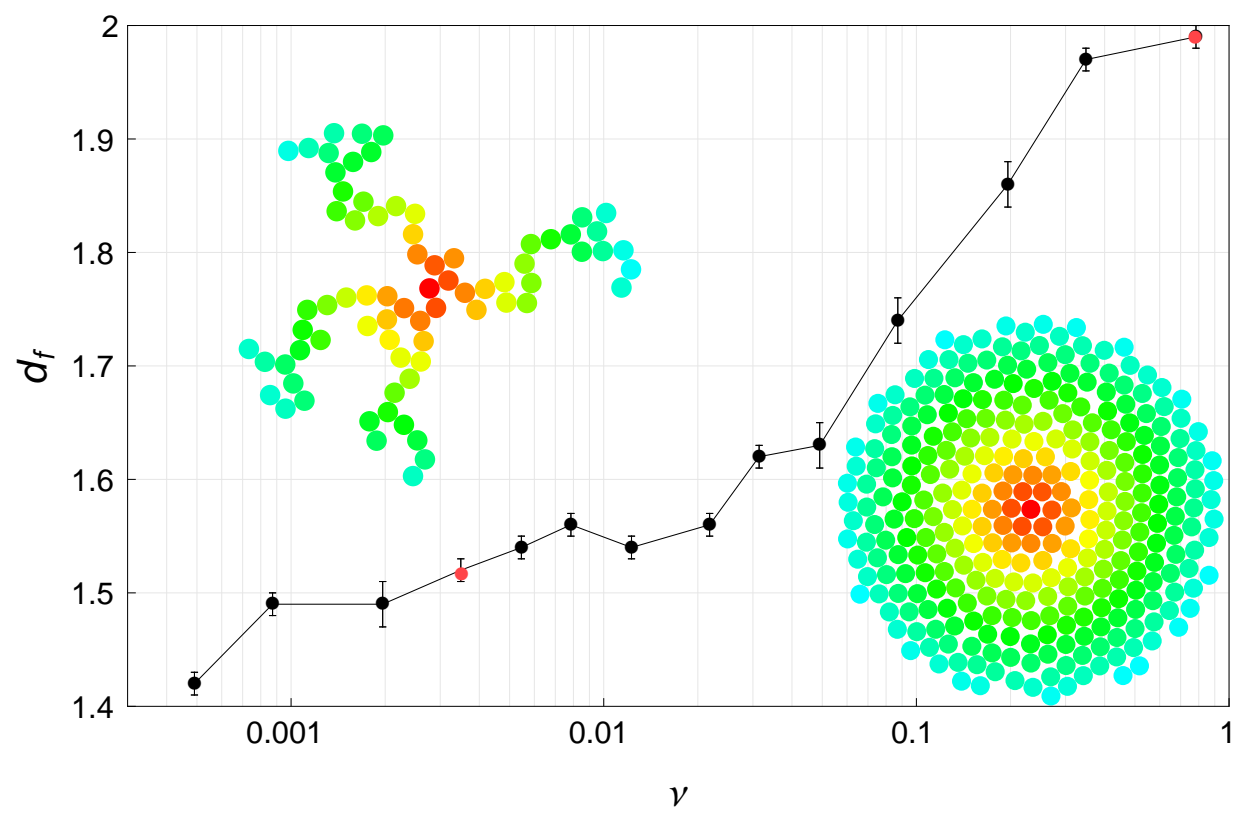

Figure 3.5: Fractal dimension as a function of packing fraction for systems with $N=10^{6}$ particles. The dots are the simulation results while the solid line is just a guide to the eye. The error bars correspond to the fluctuations associated with the measurement of $d_{f}$ and not to ensemble averages. In the inset, two examples of the structures obtained for two different densities $\nu_{\text {dilute }}=0.0035$ and $\nu_{\text {dense }}=0.784$, marked as red points on the plot. The structures are colourised from red to blue depending on the distance to the central particle. 



\section{Chapter 4}

\section{Free Cooling of Hard-Spheres with Long-range Interactions}

We study the stability of the free cooling of a granular gas of mono-disperse particles with long-range interactions. We analyse structure formation in this system by means of two independent methods: event-driven molecular dynamics with discrete potentials (in 2D and 3D) and linear stability analysis of the corresponding hydrodynamic equations (in 2D), where only the energy dissipation term is modified to account for the long-range interaction. Attractive potentials enhance structure formation while, on the other hand, repulsive potentials inhibit it, as intuition indicates. However, if the potential is weak enough, structures will develop before the repulsion has time to act. The time for this effect to take place is estimated and the modifications to the hydrodynamics modes discussed. Furthermore, for repulsive potentials, in the homogeneous regime, the cooling shows a universal behaviour when the temperature is normalised by the bounding energy. Remarkably, for low dissipation and density, the data is captured by a previously developed mean field theory for continuous potentials in 3D. The non-homogeneous regime is also studied and the effect of the potential strength in relation to the cluster formation is investigated.

\subsection{Introduction}

Granular gases are granular materials where the duration of a collision is much shorter than the typical collision time [17-24]. This situation can be obtained by either placing a dilute particle system in a micro-gravitational environment (such as parabolic flight [57]), or experimentally easier, by feeding the system with energy such that a gaseous steady state appears (e.g., by vertically vibrating the enclosure [58]). For a granular gas, in the dilute limit, binary collisions dominate over multiple collisions. Contrary to molecular 
gases, granular gases are dissipative. So the continuous loss of kinetic energy due to collision not only makes the gas cool down but is also accompanied by collective phenomena such as cluster formation or shear banding. Granular gases are subject to instabilities and cluster formation $[19,22,34,58,59]$, deviations from the Maxwell-Boltzmann velocity distribution [60,61], phase transitions [62] and the formation of vortices [19].

The hydrodynamic instability that leads to cluster formation is exclusively an effect of dissipation during collisions for the case of hard spheres [18], and thus should be enhanced by attractive potentials and diminished by repulsive potentials between the particles. Electrically charged granular media are, in nature and industrial processes, the rule rather than an exception (see Ref. [63] and references therein). Particle systems with attractive longrange interactions can be found, for example, in dry powders, electrostatic coating processes, and in space. In the latter case, huge mass distributions such as dense granular rings and disks around central bodies are affected by considerable self-gravitation [64-66].

This study is devoted to the behaviour of granular media with either (long-range) repulsive or attractive potentials in the dilute limit, and furthermore we do not consider attractive and repulsive potentials acting at the same time. Our goal is to understand how do dissipation and long-range interaction relate and what kind of dynamics produces the inclusion of both effects in a free cooling granular system.

We study the system through the stability analysis of the hydrodynamic equations for a granular fluid and compare the results with event-driven simulations. We find out that the homogeneous cooling of a granular gas, at low enough densities, and with repulsive long range interaction is universal in the sense that it does not depend on the shape of the potential. For the repulsive case the linear analysis predicts the behavior of the system, but not for the attractive case. In section 4.2 we present the $2 \mathrm{D}$ hydrodynamic equations for our system. We introduce the modified cooling rate due to longrange interaction in sec. 4.3 together with the stability analysis of the fluid equations. We introduce our simulation method in sec. 4.5 , and continue with numerical results in sec. 4.6. The phase diagram for this system is presented in 4.7. Conclusions and perspectives of future work finalise the paper in sec. 4.8

\subsection{Classical Granular Gas}

In this section we review the theory of the free cooling granular gas, what is know in the literature as Haff's law. 


\subsubsection{Free Cooling Theory}

In the homogeneous cooling state (HCS) [17] the decay of the kinetic energy $K$ is governed by the equation

$$
K(\tau) / K(0)=(1+\tau)^{-2}
$$

with the rescaled time $\tau=\left(1-r^{2}\right) t /\left(2 \mathfrak{D} t_{E}\right)$. $\mathfrak{D}$ is the dimension of the system, $r$ the coefficient of restitution, and $t_{E}$ is the initial Enskog collision rate [37],

$$
t_{E}=\frac{d \sqrt{\pi}}{2^{\mathfrak{D}} \mathfrak{D} \nu g_{\mathfrak{D}}(\nu) \sqrt{T_{g} / m}},
$$

with the initial granular temperature $T_{g}=T / m=\frac{2}{\mathfrak{D}} \frac{K}{N}$, i.e. twice the kinetic energy per particle per degree of freedom, while $T$ is the square of the velocity fluctuations. The diameter of the particle is $d, \nu$ is the packing fraction of the system and $m$ the mass of the particles. This collision rate defines a non-dimensional time $\tau=t / t_{E}(0)$ for the simulations (see below). In 2D, the pair correlation function at contact is given, approximately for low to moderate densities, by $[42,67]$

$$
g_{2}(\nu)=\frac{1-7 \nu / 16}{(1-\nu)^{2}}
$$

and in 3D by

$$
g_{3}(\nu)=\frac{1-\nu / 2}{(1-\nu)^{3}} .
$$

Improved formulae for higher density can be found in $[37,68,69]$.

\subsubsection{Hydrodynamic Equations}

The hydrodynamic equations for a granular gas are explained in detail in [70] (Chapter 2). For the sake of brevity, we present a summarised version.

The continuity equation for the mass density $(\rho=M / V)$ reads:

$$
\frac{D \rho}{D t}+\rho \frac{\partial u_{i}}{\partial x_{i}}=0,
$$

where $u_{i}$ is the velocity in the $x_{i}$ direction. Momentum conservation in absence of gravity gives:

$$
\rho \frac{D u_{i}}{D t}=-\frac{\partial \sigma_{i j}}{\partial x_{j}},
$$


where $\sigma_{i j}$ is the stress tensor. The energy balance reads:

$$
\rho \frac{D}{D t} T=-\sigma_{i k} \frac{\partial u_{i}}{\partial x_{k}}-\frac{\partial q_{k}}{\partial x_{k}}-\hat{\gamma} T
$$

where $q_{k}$ is the heat flux and $\hat{\gamma}$ the dimensional cooling rate. For the explicit formulation of the coefficients see [37,70] and Chapter 2.

The homogeneous reference solution (HCS) for a freely cooling gas is found by taking all the spatial derivatives in the hydrodynamic equations equal to zero, leaving just one non-dimensionalised equation for the temperature:

$$
\frac{d T}{d t}=-\tilde{\gamma} T
$$

where the energy density dissipation rate is $\tilde{\gamma}=-\gamma_{0} T^{1 / 2}$. The remaining fields - density and velocity in each component - remaining homogeneous and constant:

$$
\nu=\nu_{0}, \vec{u}=0 .
$$

This was the solution Haff derived from simple mechanical arguments in his seminal paper [17]. The homogeneous-state variables and coefficients are designated by a subscript " 0 " $\left(\nu_{0}, p_{0} \equiv p\left(\nu_{0}\right), s_{0} \equiv s\left(\nu_{0}\right)\right.$, etc. $)$, and the derivatives with respect to density (evaluated at $\nu_{0}$ ) are denoted as, e.g., $p_{\nu_{0}} \equiv d p /\left.d \nu\right|_{\nu_{0}}$.

\subsubsection{Cluster Instability}

The homogeneous cooling state, however, is not always a stable solution for the system. For the hard-sphere potential, when the system size is large enough (at a given dissipation), the homogeneous cooling becomes instable and shear and clustering modes appear in the system. For the attractive potential we expect that the cluster instability will be always enhanced, and on the contrary, for the repulsive case, impaired.

The spontaneous formation of clusters in a force free cooling granular gas can be understood by simple arguments [18,71]: consider density fluctuations in an otherwise homogeneous granular gas. In denser regions the particles collide more frequently than in more dilute regions, therefore, dense regions cool faster than dilute regions and the local pressure decays in these colder regions. The resulting pressure gradient causes a flux of particles into the denser region, which leads to further increase of the density. Hence, small fluctuations of the density are enhanced, which leads to the formation of clusters. 
Since the simulation and the hydrodynamic equations can be scaled to arbitrary units and non-dimensionalised, we will take both the particle radius and initial temperature to be one. The potential strength is scaled by the initial temperature and hence is dimensionless. Figure 4.1 shows snapshots for the same initial conditions after $7 \times 10^{5}$ collisions for an event-driven simulation with long-range potential. We use relative week potentials with $\phi=-10^{-5}, 0,10^{-5}$, that is, attractive, neutral, and repulsive. The three systems present similar clusters but the evolution is different for each one of them. The following section introduces the theoretical approach we will use to study these differences.
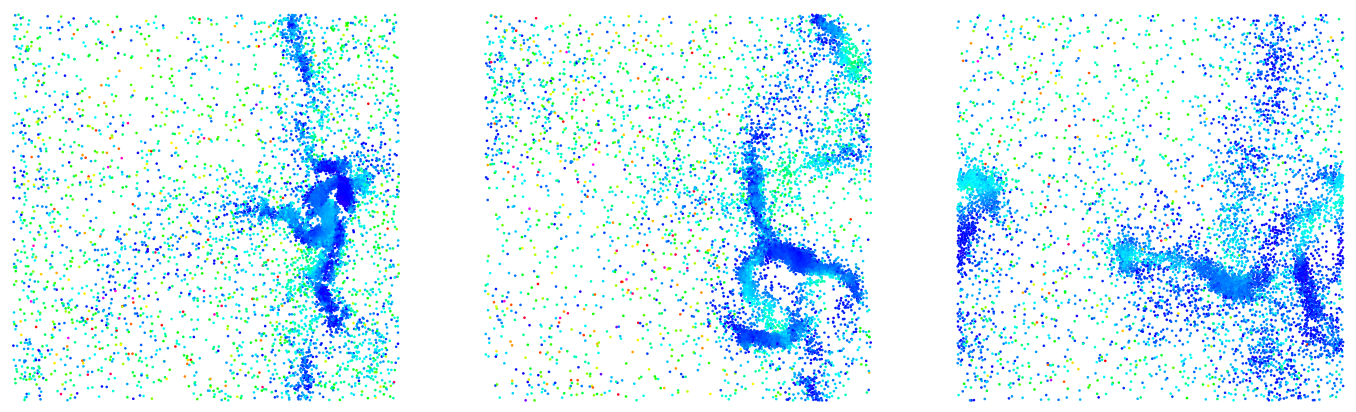

Figure 4.1: Snapshots after $7 \times 10^{5}$ collisions for systems with $\phi=$ $-10^{-5}, 0,10^{-5}$, from left to right. $N=6400, \nu=0.0578$ and $r=0.65$. The color code indicates the kinetic energy per particle, from blue (slow), to yellow (average) and red (fast).

\subsection{Long-range cooling: modification of the dissipation rate}

Haff's law is valid only for particles with hard-core interaction. For long range interaction, Müller and Luding [72,73] predicted, using a modified pseudoLiouville operator formalism, a reduced cooling rate due to the repulsive forces and an increased rate due to attractive forces.

In this theory, the ratio of the long-range potential at contact, $\phi$ to the temperature is the control parameter,

$$
\Gamma=\frac{|\phi|}{T}
$$

It must be noted that since the theory is a mean field theory, the shape of the potential does not enter in the formulation, only its value at contact [72].

Since the cooling rate is modified, the transport coefficients of the system will be modified accordingly. We will focus only on the change produced 
in the dissipation rate, since it is the dominant term in the dynamic of the system. Namely, the dissipation coefficient is modified as follows:

$$
\tilde{\gamma}=\left\{\begin{array}{lr}
\gamma_{0} & : \text { Haff } \\
\gamma_{0} \exp (-\Gamma) & : \text { Repulsive } \\
\gamma_{0}(2-\exp (-\Gamma)) & : \text { Attractive }
\end{array}\right.
$$

These modifications were derived for 3D systems by computing the average effect of a long-range potential in the collision frequency of a granular system with a pseudo-Liouville operator approach. We will use them directly in 2D accounting for the different dimensionality in the pair correlation function at contact and the numerical factors in the Enskog collision time. This is justified since the integration of the Liouville operator considers collisions in a plane due to angular momentum conservation, and hence only the prefactor is different between 3D and 2D, while the functional form in $\Gamma$ remains identical.

\section{Predicted Homogeneous Cooling}

The homogeneous cooling with long range interaction is given by Eq. (4.5). It was numerically solved (we used Mathematica 8) and Fig. 4.2 shows the evolution of the temperature for three cases: attractive $(\phi=-1)$, repulsive $(\phi=1)$ and no long-range interaction (Haff's law, $\phi=0)$.

Physically, Fig. 4.2 says the following: In Haff's case, the dynamics of the systems becomes slower as time advances, making the dissipation slower, and so on, as long as the system is homogeneous [17]. In the presence of long-range forces, the system can have at least two different regimes: at the beginning, the thermal energy is larger than the repulsive/attractive energy, its effect being negligible. As the system cools down, the repulsive/attractive barrier will start to be felt, and the cooling will be consequently modified.

For the attractive force the prediction says that once the particles start to feel the attraction they will dissipate energy faster but nevertheless will retain the power law of the dissipation and keep the homogeneity.

\subsection{Stability Analysis}

In this section we present the stability analysis for the modified cooling rate due to the long-range interaction in $2 \mathrm{D}$. We first write down the linearised system of equations and then the eigenvalue equations for the shear mode. The explicit form of the other modes can be found in $[19,70]$.

In what follows, we assume that the base state of the system is Haff's law. This is valid only when the long range potential is small compared with 

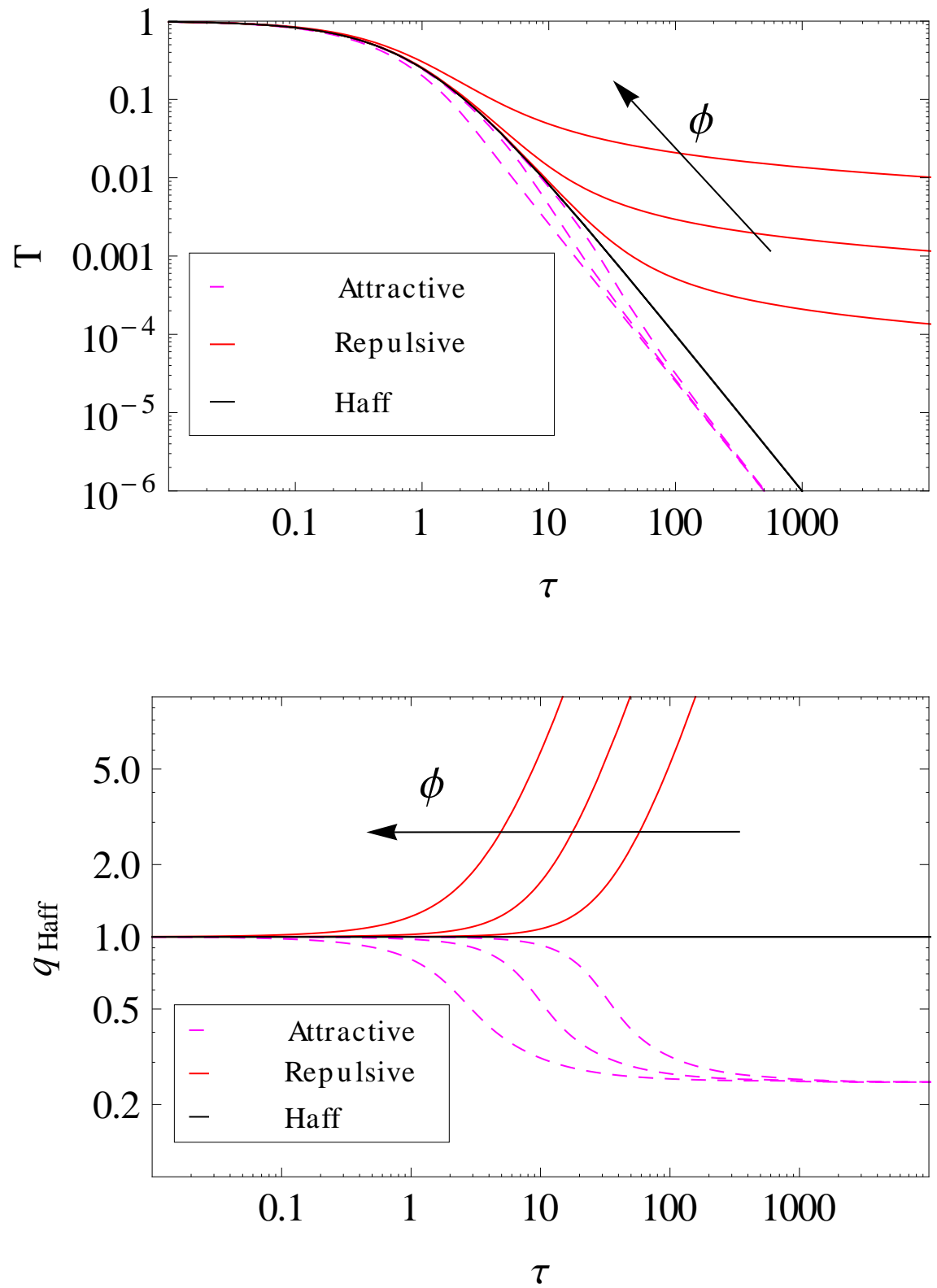

Figure 4.2: Cooling of granular systems. Top: temperature evolution for hard-spheres (Haff's law, solid black), repulsive interaction and attractive interaction as given by the numerical solution of the temperature equation (4.5) considering the dissipation term as in Eq. (4.8) for different values of phi $=10^{-3}, 10^{-2}, 10^{-1}$. Bottom: quality factor $q_{\text {Haff }}=T / T_{\text {Haff }}$ for the same long range potentials as on the top plot.

the temperature. The reason for this choice is the following. In the attractive case, once the temperature and the repulsive potential are comparable, the system becomes homogeneous to any perturbation, so it is irrelevant to make stability analysis on this state for we already know the answer. On the 
contrary, for the attractive case once the attractive potential is comparable to the temperature the systems develops clusters. The validity of the hydrodynamic equations in this case is, at least in the present form, questionable.

\section{Linear Equations}

Since the stability analysis here is the same as the one done in Chapter 2, we refer the reader there for the details and explicit formulation of the transport coefficients.

In the following, we study the linear stability of the system in its new time variable, $\tau$, since then the equations can be written in the form $d \mathbf{Z}^{\prime} / d \tau=$ $F\left(\mathbf{Z}^{\prime}\right)$, with the vector of perturbed fields $\mathbf{Z}^{\prime}$. Since we work in the approximation $\phi \ll 1$, the equations become time-dependent only via $\Gamma$.

If we would keep the physical time as our variable, the equation for the temperature would be explicitly time-dependent.

In order to linearise the hydrodynamic equations about the homogeneous but time-dependent solution (4.1), the fields are split into their homogeneous and their perturbation (primed) parts:

$$
\nu=\nu_{0}\left(1+\nu^{\prime}\right), u=f u^{\prime}, v=f v^{\prime}, T=f^{2}\left(1+T^{\prime}\right),
$$

where $\nu$ is the volume fraction, $u(v)$ the bulk velocity in $x(y)$ direction, and the primes indicate the perturbations. The primed quantities define $\mathbf{Z}^{\prime}$, and the factor $f$, the collision frequency, is introduced for later convenience.

The final goal of this section is to examine the linear stability of small perturbations of the form $\exp (i \mathbf{k} \cdot \mathbf{x})$. Without loss of generality, consider perturbations only along the $x$ direction so that the derivatives in the $y$ direction vanish. Taking the perturbations up to first order gives four coupled equations. By inserting the fields into Eqs. (4.3), (4.4) and (4.5):

$$
\begin{aligned}
\nu_{t}^{\prime} & =-f u_{x}^{\prime} \\
\nu_{0}\left(f u^{\prime}\right)_{t} & =-p_{x}^{\prime}+\left(\mu_{0}+\zeta_{0}\right) f u_{x x}^{\prime}, \\
\nu_{0}\left(f v^{\prime}\right)_{t} & =\mu_{0} v_{x x}^{\prime} \\
\nu_{0}\left(f^{2} T^{\prime}\right)_{t} & =-p_{0} f u_{x}^{\prime}+\kappa_{0} f^{2} T_{x x}^{\prime}-\gamma_{0} f^{2} T^{\prime}-\gamma^{\prime} f^{2}-2 \nu_{0} f f_{t} \nu^{\prime},
\end{aligned}
$$

where the explicit expressions for the non-dimensional transport coefficients in the homogeneous state, $p_{0}, \mu_{0}, \zeta_{0}, \gamma_{0}$, and their perturbed values $p^{\prime}$ and $\gamma^{\prime}$ can be found in Ref. [19] and in Sec. 2.3.4.

For the classical stability analysis, one changes variables to intrinsic time scale of the system, given by the collision frequency, $\tau$, using the identities $f=\exp \left(-\epsilon^{2} \tilde{\gamma} \tau\right), d \tau / d t=f / s_{0}$, and scaling lengths by density as $X=$ $\nu_{0} x$ and thus wave numbers as $K=\nu_{0}^{-1} k$. In our case, however, $\tilde{\gamma}$ is not 
a constant. We nevertheless neglect this dependence and simply add the modified dissipation rate to the classical eigen-value equations for stability, replacing $\tilde{\gamma}$ by the corresponding long-range term from (4.8). The validity of this assumption will be confirmed later by the simulations.

\section{Shear Mode}

Since the shear mode is decoupled from the other modes in the hydrodynamic equations, the method we use can be easily explained by this example. The equation for the shear mode in the non-dimensional coordinates for time and space reads:

$$
\nu_{0}\left(f v^{\prime}\right)_{\tau}=\mu_{0} v_{x x}^{\prime},
$$

with $\nu_{0}$ the non-perturbed density and $\mu_{0}=$ the shear viscosity (see Sec. 2.3.4). The approximation we make now consists of introducing the new dissipation rate taken all the other variables as constants.

The standard method for stability analysis [19] considers a plane wave as the solution for the hydrodynamic modes and studies the growth rate of an arbitrary perturbation. If the growth rate is positive, the mode is instable. Assuming that $v^{\prime} \propto \exp (-\sigma \tau+k x)$, the dispersion relation for the shear mode in Haff's case reads

$$
\sigma=-\mu_{0} k^{2}+\epsilon^{2} \tilde{\gamma}(T)
$$

This approximation is formally equivalent to Eq. (9) in Ref. [74]. As noted before, for the long-range case we assume that the homogeneous cooling is still the base state since the long-range potential has a small amplitude, i.e. $\Gamma_{0} \ll 1$. Hence, the collision frequency does not appear to first order and the exponential terms enters only on the dissipation rate: $\tilde{\gamma}(T)=\gamma_{0} e^{-\Gamma}$.

Figure 4.3 shows the evolution for ten different wave numbers of the shear mode, from $k=1, \ldots, 10$ in even intervals, solved numerically. The attractive case (left) shows that for $k$ small enough the system is instable, while for the repulsive case all the wavelengths are eventually suppressed and the system becomes stable and homogeneous.

\section{Critical wavelength for the other modes}

As we saw in Chapter 2, the other hydrodynamic modes are linear combinations of the density, temperature and transversal velocity. They can be solved numerically but for the sake of simplicity we are going to focus only on the critical wave number as a function of density for different $\Gamma$ and draw the physical consequences of it.

Instead of looking at the dispersion relation for the other modes, we 

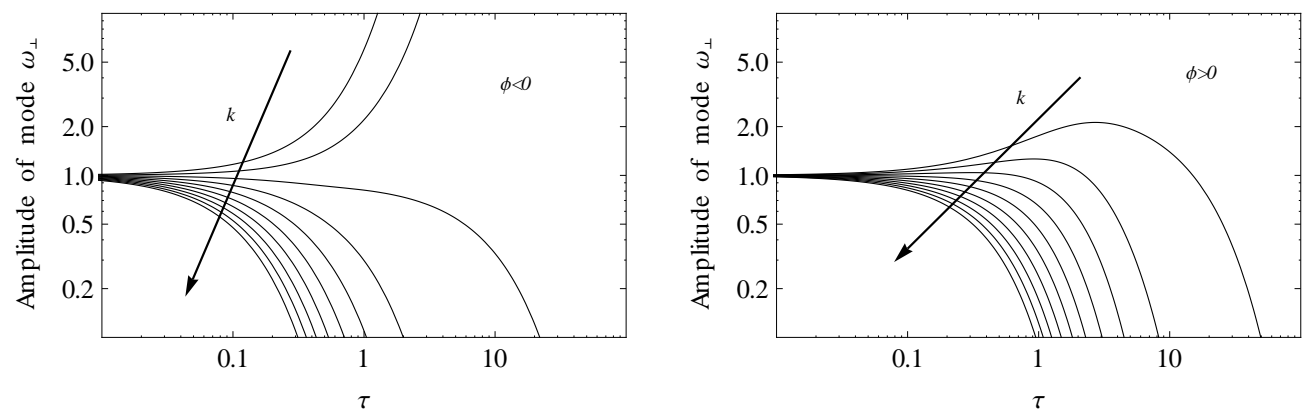

Figure 4.3: Evolution of shear mode for different $K$. (We have considered $\epsilon^{2} \gamma_{0}=\mu_{0}=1$ for the sake of simplicity; for different restitution coefficients and density the plot is scaled but the form remains the same. In the left, $\phi=-1$ while in the right $\phi=1$.

focus on the dependence of the critical wave lengths on density [19]. This is a clearer way to picture how the long-range potential affects the stability of the system.

Figure 4.5 shows the different criteria for instability for attractive and repulsive potentials at different $\phi$ for $r=0.9$. Each line represents the smallest $K$ at which each mode becomes instable. As $K$ is decreased, the shear mode is the first to become unstable. As $K$ is decreased further, the stability condition for the granular heat mode is violated.

If we move now downward in the plots, we see that in the repulsive case the critical $K$ moves upward as $\phi$ increases, while the repulsive case is exactly the contrary: the critical $K$ decreases as $\phi$ increases. Furthermore, for the attractive case, qualitative changes appear at around $\phi \sim-1$, while for the repulsive case they appear at $\phi \sim 0.3$.

This make a quantitative difference between the attractive and the repulsive case: while the attractive case becomes significantly different at $\phi \sim 1$, the repulsive potential becomes active much earlier, and furthermore, when the thermal energy and the potential are comparable, all the disturbances are blushed away and the system remains homogeneous independent of the restitution coefficient and the density of the system.

In the next section we will see how this is reflected by the simulations.

\subsection{Event-Driven simulations}

The simulations used to prove the theoretical predictions in Ref. [72] were done with continuous potentials, which make them computationally expensive and time consuming. However, the theoretical description does not consider the shape of the potential but only its value at contact. Therefore, we 

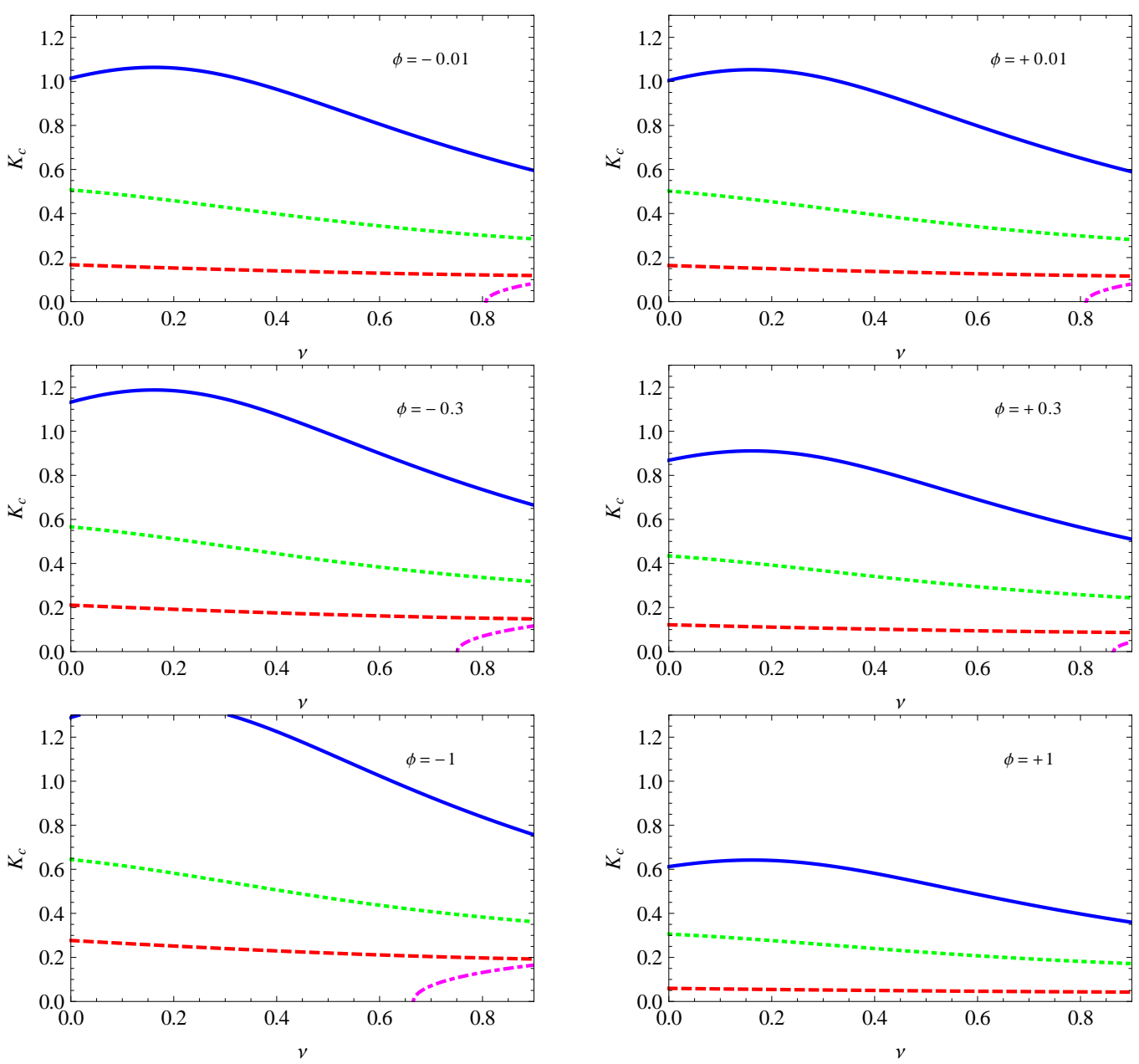

Figure 4.4: Critical wave numbers as a function of density for attractive (left) and repulsive (right) potentials at $\phi= \pm 0.01$ (upper row), $\phi= \pm 0.3$ (middle) and $\phi= \pm 1$ (lower row). Shear-mode in solid-blue and heat-mode (dotted-green), together with the other stability criteria (see [19]): $b>0$ (dashed-red) and $a b-c>0$ (dash-dotted-magenta).

use much faster discrete potential simulations where the range of attraction is finite.

Event-driven simulations have been widely used to study granular gases $[19,22,71]$, and have shown to capture the correct behavior. In what follows we present the details of the simulations.

\subsubsection{Discontinuous potentials}

Discrete potentials, such as the hard sphere model, have an important advantage over more complex "soft" potentials. Between collisions the spheres or molecules experience no forces and travel on ballistic trajectories. The dynamics can be solved analytically, and the integration of the equations of mo- 
tion is processed as a sequence of events rather than fixed, small time-steps. Current event driven molecular dynamics algorithms are quite advanced and allow the simulation of large systems for the long times required to extract accurate transport properties and study, e.g., the evolution of clusters $[70,75]$ over many orders of magnitude.
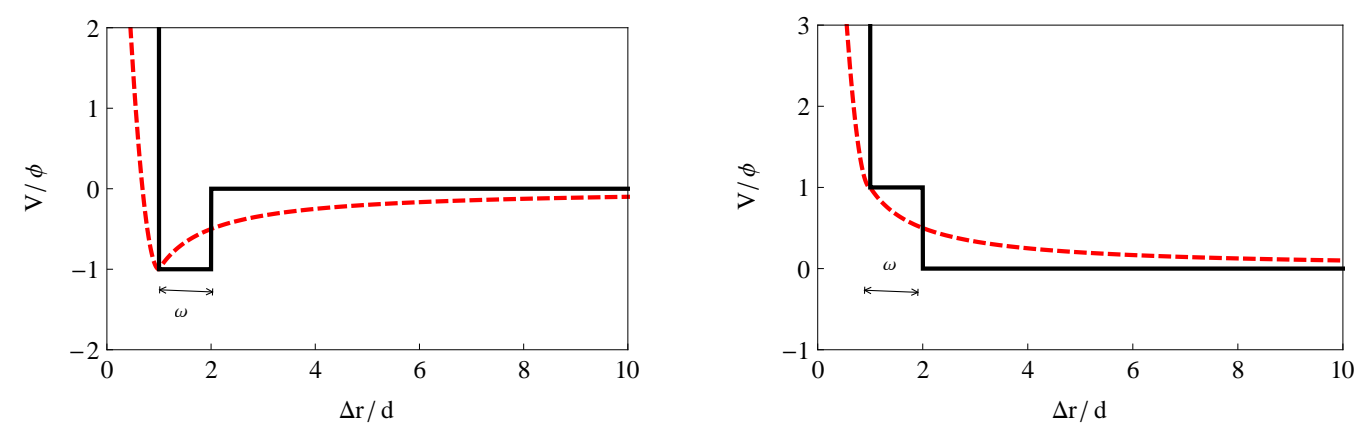

Figure 4.5: Plot of the potential energy for both continuous and discontinuous models with attractive (left) and repulsive (right) potentials as a function of the inter-particle distance $r$.

For our simulations we use a two-level potential. The core is a hard sphere and there is a well (or barrier) at a distance $\omega=1.5$ (for all simulations unless otherwise noted) and of amplitude $\phi$. The core is a hard sphere potential and the relative velocity in a collision is updated only in the normal direction according to

$$
\mathbf{v}_{i j}^{\prime}=\mathbf{v}_{i j}-(1+r)\left(\mathbf{v}_{i j} \cdot \mathbf{r}_{i j}\right)
$$

where $\mathbf{r}_{i j}$ is the distance between the particles, $\mathbf{v}_{i j}$ is the pre-collisional relative velocity, and the prime indicates a post-collisional quantity. At crossing the well (barrier) the particles loose or gain energy instantaneously depending on the direction of the collision. The velocity after the crossing can be written as:

$$
\mathbf{v}_{i j}^{\prime}=\sqrt{v_{i j}^{2} \pm 2 \phi / m}
$$

with $\left|\mathbf{v}_{i j}\right|=v_{i j}$, and the sign depends on whether the potential is attractive or repulsive and whether the collision is outgoing or incoming. For an incoming collision, in the attractive (repulsive) case, the particles gain (loose) energy. For the outgoing collision this is reversed. How the sign and magnitude of this potential affect the macroscopic evolution of a free-cooling gas is the subject of the rest of the study. 


\subsubsection{System and Preparation}

The simulation consists of a system of $N$ particles in a square box of side length $L$ with periodic boundary conditions. Particles are mono-disperse with diameter $d$ and mass $m$. The packing fraction of the system is given by $\nu=N \pi d^{3} /\left(6 L^{3}\right)$ in $3 \mathrm{D}$ and by $\nu=N \pi d^{2} /\left(4 L^{2}\right)$ in $2 \mathrm{D}$. The simulations were carried out in DynamO, a free and open-source event-driven code [13].

\section{Initial Conditions}

The initial state is prepared as follows: (1) Start with a square lattice (2D) or HCP packing (3D), uniform random velocities and a fixed $\phi$. (2) Let the system equilibrate: each particle collides at least 100 times elastically until a homogeneous regime is reached. This is tested looking at the distribution of velocities and confirming that is very close to a Maxwellian. (3) Once thermalised, the velocities are scaled so $T_{0}=1$ and dissipation is turned on. From here, the system is let to freely cool down.

\section{Simulation Units}

The simulation runs a non-dimensionalised system. The units of length, mass and time are set such that, $d=1, m=1$ and $T_{0}=1$. Since we are interested in the perturbative case, the potential, $\phi$, is varied between $10^{-3}$ and $10^{-5}$ for both, attractive and repulsive cases.

\subsection{Numerical Results}

In this sectiont we present first the results for the attractive regime, followed by the repulsive case.

\subsubsection{Attractive forces}

In this subsection we analyse data for cooling with attractive potentials of different intensity and for different restitution coefficients. The objective is to understand how the dynamics is influenced by these two factors and how they interact. We focus on the evolution of three aspects: the temperature, i.e. cooling, the velocity distribution of the particles, and the cluster structure and size distribution.

The initial state for different systems is always the same: the distribution of particles is homogeneous, with a Maxwellian velocity distribution, and, hence, the cooling is well described by Haff's law. As time passes dissipation and the attractive potential will have time to act and modify this picture giving rise to clusters. 


\section{Cooling}

The theoretical prediction from Müller and Luding [72] for the cooling rate is an homogeneous state with a modified dissipation rate, namely, twice as large. From Eq. (4.8), we have that $\lim _{t \rightarrow \infty} \gamma=2 \gamma_{0}$. Furthermore, systems with different $\phi$ will deviate from Haff's law at different times; the smaller $\phi$ the more time it takes to deviate, however, the functional form remains the same.

Fig. 4.6 shows the temperature (normalised by Haff's law) for systems with $\phi=10^{-3}, 2 \times 10^{-3}, 5 \times 10^{-3}, 8 \times 10^{-3}, 10^{-4}$ and low dissipation so the cooling is homogeneous. The cooling is well predicted by the theory only for the initial deviation from Haff's law but there is no agreement after that; simulations dissipate more energy than predicted. Interestingly, the simulations do seem to collapse to a power law of the same slope but with different scaling factor. This can be seen around $\tau \simeq 100$ where all the curves collapse at a value close to $q_{\mathrm{Haff}} \simeq 1.5$. Due to the long time it takes to simulate the clusterised state, we do not have data on the long term regime.

The time evolution for these low-dissipation systems can be seen in Fig. 4.7. Once the attractive force is larger than the thermal fluctuations, the system develops clusters. The structure of these clusters is typical for a cluster-cluster aggregation process, with an exponential decay on the cluster size (see below).

\section{Velocity Distribution}

The temperature alone is not a good indicator of the structure formation process since this also depends on phi. A better indicator is to look for the deviation of the velocity distribution from a Maxwellian. For this, we focus on the evolution of the kurtosis $\beta_{2}=\mu_{4} / \mu_{2}^{2}$, where $\mu_{i}$ denotes the $i$ th central moment (and $\mu_{2}$ in particular the variance) as a function of $\Gamma$. When the $\beta_{2} \simeq 3.108$ the system is homogeneous. (It most be noted that since the system has a finite number of particles, the kurtosis does not reach the theoretical value but fluctuates near to it.) As soon as clusters appear in the system, the velocities of the particles in the cluster are more correlated and hence the distribution of velocities deviates from the Maxwellian towards higher values of $\beta_{2}$.

By studying the evolution of the system as a function of $\Gamma$ one is looking at the same time at the temporal evolution of the system. The advantage of looking at $\Gamma$ instead of at the proper time of the system is that different coefficient of restitution can be studied in the same plot without shifting the he axis. For different coefficients of restitution and phi the temporal 


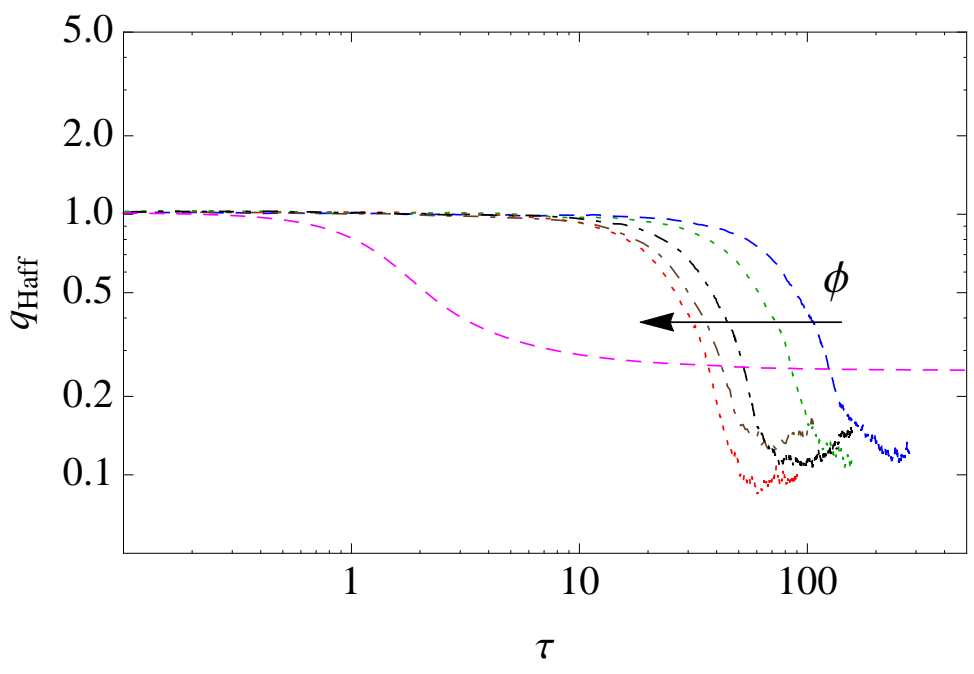

Figure 4.6: Temperature evolution normalized by Haff's law with attractive potential for $r=0.99, \phi=10^{-3}, 2 \times 10^{-3}, 5 \times 10^{-3}, 8 \times 10^{-3}, 10^{-4}$ together with Haff's law (solid-black) and the theoretical prediction for $\phi=1$ (dashed magenta). The onset of cooling in the simulations is well predicted but the later cooling rate is overestimated, the magenta line runs noticeably on top of the simulations.

$$
\Gamma^{-1}=1
$$

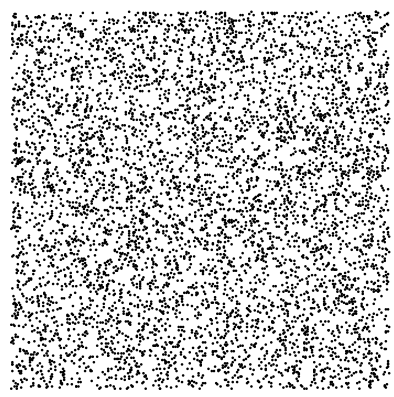

$\Gamma^{-1}=0.1$

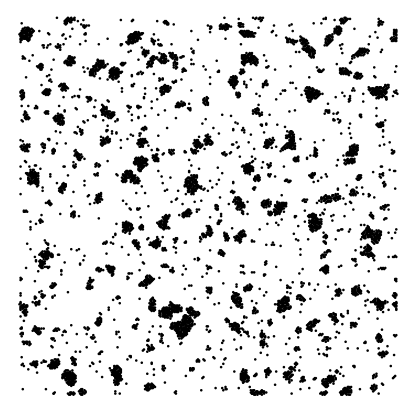

$\Gamma^{-1}=0.003$

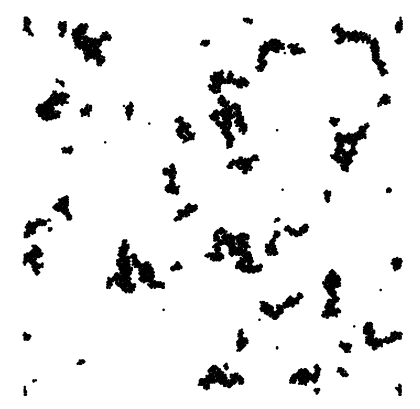

Figure 4.7: Simulation snapshots at different $\Gamma$ (time increases from left to right) for a considerable attractive potential $(\phi=1)$ for $N=6400, \nu=$ 0.0578 and $r=0.99$. This corresponds to a system equivalent to the lower dashed line in Fig. 4.8 but with a larger attractive force. 
evolution can spread over several orders of magnitude. With $\Gamma$, all these curves collapse in a single region.

Figure 4.8 shows the kurtosis during the cooling of three systems with attractive potentials for different restitution coefficients and potential strength for a fixed density. If the cooling is homogeneous (solid and dashed lines), the deviation from the homogeneous state is due solely to the attractive potential and sets up when $\Gamma^{-1}$ is smaller than one. When the cooling is not homogeneous, the system deviates from the Maxwellian distribution of velocities before the attractive potential has time to act.

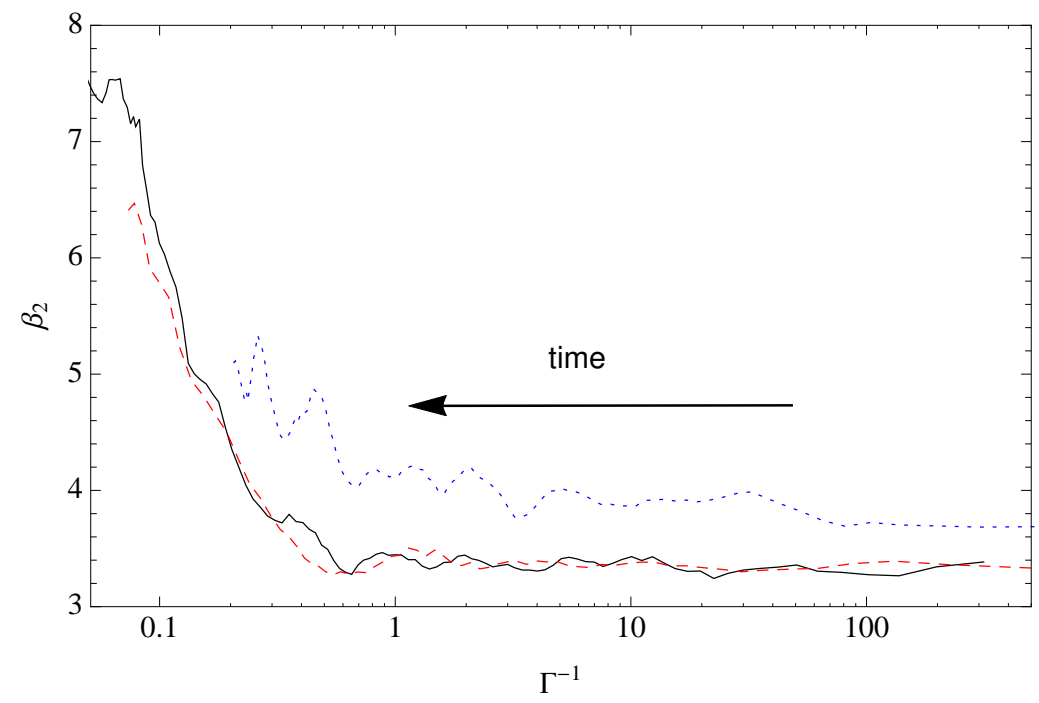

Figure 4.8: Kurtosis of the velocity distribution as a function of $\Gamma$ for systems with attractive potential. Different $\phi$ collapse on the same curve as long as the coefficient of restitution is large enough. When the coefficient of restitution goes below the critical value given by HCS, the system develops dissipative clusters before the attractive cooling sets in. The bottom lines correspond to an evolution as in Fig. 4.7, while the upper line corresponds to an evolution as observed in Fig. 4.9.

\section{The cluster structure as a function of $\phi$}

Despite similar final states for each system with dissipation and attractive force, there are a number of distinctive characteristics depending on how strong the attractive potential is compared to the initial kinetic energy of the system.

Roughly, we divide the phenomenology in two different regimes quantified by $\Gamma$ : comparable $(\Gamma \sim 1)$ and small $(\Gamma \ll 1)$ long-range effects. In the first regime, the attractive potential is stronger than the kinetic energy and hence when particles collide, due to the dissipation, they tend to remain bound 
together creating clusters in a homogeneous way. The second regime is one where the attractive potential is weak enough to let the cluster instability appear in the system. Once a cluster occurs, the attractive force binds it together and makes the dissipation to be stronger than in an equivalent cluster of hard spheres. The regime $\Gamma \gg 1$ is not considered, since it resembles the limit of cluster-cluster aggregation and the dissipation has no role to play there.
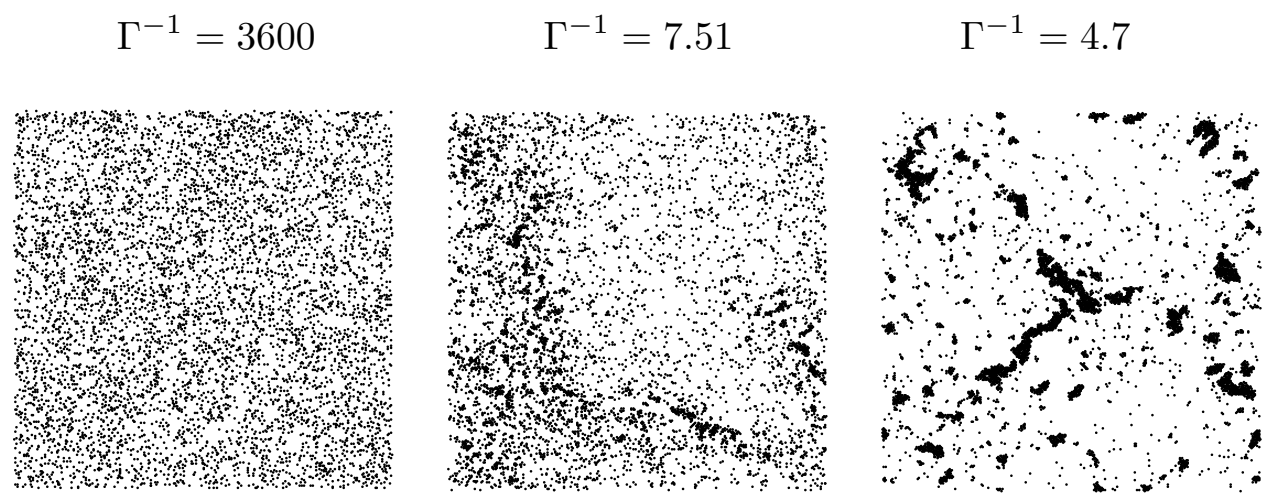

Figure 4.9: Simulation snapshots at different $\Gamma$ (time increases from left to right) for a weak attractive potential $\left(\phi=10^{-4}\right)$ for $N=6400, \nu=0.0578$ and $r=0.6$. This correspond to an evolution as the one depicted in Fig. 4.8, upper dotted line.

Figures 4.7 and 4.9 show snapshots of a system for different number of collisions and a different restitution coefficient in each figure. In the first case, with a relatively strong potential and low dissipation, the cooling is homogeneous until $\Gamma \simeq 1$ when clusters start to appear homogeneously, in a way similar to the cluster-cluster aggregation process. Fig. 4.9 shows snapshots for the same system but with an increased dissipation, $r=0.6$ and reduced attractive potential, $\phi=10^{-4}$. This gives enough time for dissipation-induced clusters to appear first. Once a cluster appears, since the relative velocity of the its particles is smaller than the thermal fluctuations, the attractive forces come into play, increasing the cooling inside the cluster. The effect of this is that there is no energy left to break the cluster, and once it forms, it will remain in the system with roughly the same shape, thus creating a non-homogeneous clusterisation. This can be seen from the cluster size 
distribution and its temporal evolution.

Figure 4.10 shows the cumulative distribution function (CDF) for the cluster size distribution for the two final snapshots of Figs. 4.7 and 4.9. In the homogeneous case (red dots) the distribution of cluster sizes is similar to a Poisson distribution: most of the clusters have the average size and there are fewer clusters with large sizes. On the other hand, for the nonhomogeneous case (black squares), most of the clusters have few particles, and there is one big cluster that has most of the remaining particles in the system. This can be readily appreciated by looking at the largest cluster for each simulation. In the homogeneous case, the largest cluster has around a hundred particles, while for the homogeneous case, the largest cluster is ten times bigger.

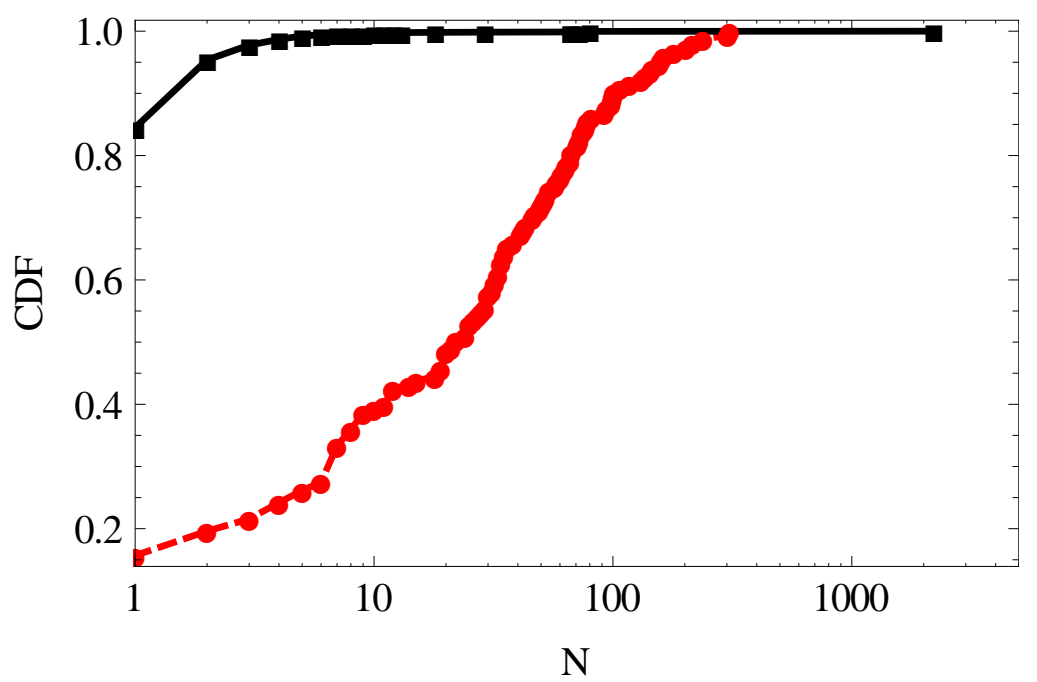

Figure 4.10: Cumulative cluster size as a function of cluster number for systems similar to those in Figs. 4.7 (red dots) and 4.9 (black squares). In the homogeneous case (dots) there are plenty of average size clusters, while in the in-homogeneous case (squares) the "winner takes it all".

\section{Attractive systems are always instable}

The reason for this behavior is that hard-spheres with attractive long-range interactions present condensation [76]. Indeed, for the elastic case, there is a region of temperatures where the liquid and gas phases can coexist. Consequently, for a homogeneous gas of cooling hard-spheres with long-range attraction, the temperature eventually reaches the critical point where the liquid phase becomes stable, and then clusters develop. These clusters will grow due to the inelasticity of the particles to eventually reach a single cluster composed of all the particles of the system. In this way, the free cooling of 
hard-spheres with long-range attraction is always instable to cluster formation disregarding the size of the system. However, the clusterisation process can be, in turn, homogeneous or inhomogeneous. It most be noted that the term homogeneous is scale-dependent: once the energy of the system is low enough particles merge into pairs breaking the local homogeneity, but remaining homogeneous in larger scale that comprises several small clusters.

This makes a fundamental difference with the free cooling of hard-spheres, where the clustering instability depends on the wavelength of the perturbation; if the system is small enough, the cooling will be always stable. We proved this by realizing small $(N \approx 100)$ simulations of attractive elastic particles - the phase separation always appeared independent of the system size. Furthermore, even for two particles, if the temperature is low enough, they will eventually find each other and merge into a cluster. Thus, since the phase separation does not depend on the system size, it always appears in a free cooling system when the temperature gets low enough $(\Gamma \approx 1)$.

\section{Comparison with wet granulates}

Our model can be compared to the cooling of wet granulates $[77,78]$. Ulrich et al. studied the cooling of a very simple model for the interaction of two wet grains, which only takes into account the essential features of a capillary bridge: hysteresis and dissipation with a well-defined energy loss. Cooling is controlled by the probability for a bridge to rupture and hence logarithmically slow in the long time limit, when a percolating structure has been formed. In contrast to theirs, our model has the dissipation occurring at collision and conserves energy at the crossing of the energy barrier (dissipation takes place at the "liquid bridge breaking" in Ulrich's model). This two microscopic differences may have a great influence in the macroscopic behavior of the system. As we have seen, our model develops inelastic clusters, contrary to Ulrich's which is homogeneous. This can, however, be due to the strong attraction they use as initial condition $(\Gamma=1)$. A detailed comparison of the two models is beyond the scope of this study; however, it remains as an interesting open question to investigate in the future.

\section{Effects of higher density}

For the continuous potential of Ref. [72], the cooling of denser systems was shown to be not predicted by the dilute limit theory. This was due to the multiparticle interaction that occur in dense systems with long-range coupling. To see if the discrete potential reproduces this behaviour, we realised simulations analogous to the ones in Müller and Luding's paper [72].

One of the most notable features of the continuous potential simulations is 
that the cooling is not monotonically decreasing: the system phase-separates and the geometrical rearrangements produce an increase in the kinetic energy of the system. We did not observe this increase in the temperature for the simulations with a well width of $\omega=1.5$ despite seeing the phase separation. Hence we decided to vary the potential width to see if this long-scale rearrangements are recovered with discontinuous potentials. Figure 4.11 shows the energy for dense systems with different well widths $\omega$ in 3D. For this density, $\nu=0.157$, all the systems present a phase transition below a critical temperature: the homogeneous system becomes instable and the system phase-separates in a liquid and a gaseous (almost vacuum) region. This is independent of the well width $\omega$. However, as the well becomes wider, the phase separation changes qualitatively, from a percolating system with big bubbles to a system made of one big drop. For different $\phi$ the qualitative evolution is similar, only shifted to the corresponding temperature (data not shown). The discrete system also presents a peak in the kinetic energy if $\omega$ is large enough. In this case, the qualitative change from a cooling that is strictly monotonous to one that presents a peak, occurs around $\omega_{p} \approx 2.25$. It must be noted that since the strength of the potential was kept constant, the "bump" in the temperature shifts to earlier times since the potential energy of the system increases too when increasing $\omega$.

In these systems where the density is so large that the particles' interactions are not binary anymore, the theory does not predict the cooling behavior at all. However, the physics - at least the increase in the temperature due to geometrical rearrangements - is recovered with a discrete potential.

\subsubsection{Repulsive forces}

Intuition tells us that in the repulsive case there are two regimes depending on whether the original hard-sphere system presents a homogeneous or nonhomogeneous cooling. For the homogeneous cooling situation it is obvious that the repulsive potential will not enhance the clustering and the cooling will remain homogeneous. In the non-homogeneous case, the repulsive forces will act against the cluster formation since they tend to separate particles. However, one can expect that if the potential is weak enough, it will not affect the formation of clusters, at least temporarily before, eventually, the temperature drops under the repulsive energy and the clusters are eliminated. Quantifying this statement is the subject of the following subsections.

\section{Homogeneous Cooling}

Contrary to the attractive case, the mean field theory for repulsion is in great agreement with the simulations. The density remains homogeneous 


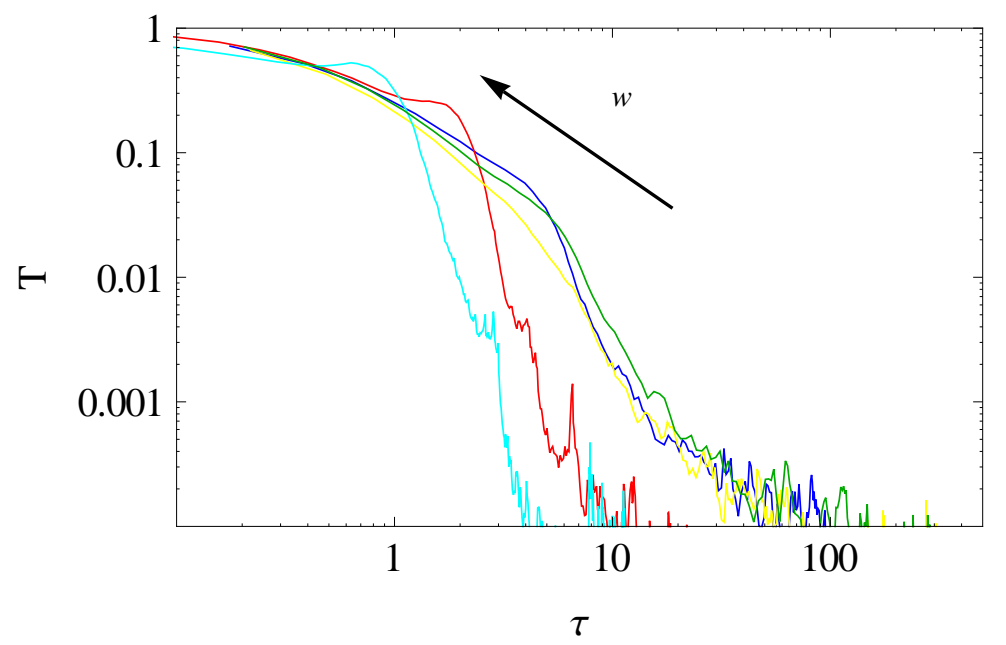

Figure 4.11: Cooling for dense $(\nu=0.157)$ systems with large dissipation, $r=0.85$ and different potential width $w=1.1,1.5,2,2.5,3$ but fixed $\phi=0.1$. As the well becomes wider, the kinetic energy presents a peak due to largescale reorganization.

and the temperature follows Eq. (4.5). This is a strong result as there are no free parameters in the theory, everything is determined by the potential at contact. Fig. 4.12 shows the temperature for two systems, in both 3D and $2 \mathrm{D}$. The prediction works equally well for any $\phi$, the only difference is that the cooling is shifted to earlier/later times depending on the strength of the potential. For 3D, the modification in the cooling rate fits perfectly the cooling of the system as long as it remains homogeneous. For 2D systems there is an appreciable deviation for long times: the theory under-predicts the cooling.
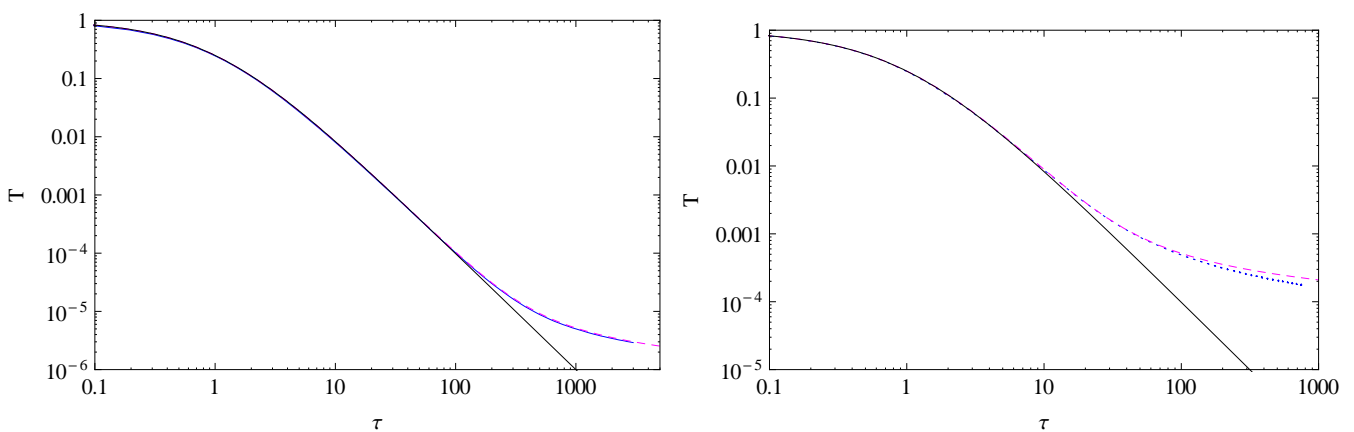

Figure 4.12: Cooling in 3D and 2D for the homogeneous case with $\phi=10^{-3}$ and $\phi=10^{-5}$, left and right respectively. The $2 \mathrm{D}$ system is denser $\left(\nu_{2 D}=\right.$ 0.057 versus $\nu_{3 D}=0.0052$ ) and that may cause the small difference for large $\tau$ between the theory (magenta dashed line) and the simulations (blue dotted line). The black solid line is Haff's cooling state for reference. 


\section{Non-Homogeneous Cooling}

A more interesting case is when the system is large/dissipative enough to present clusters. If the repulsive potential is weak enough, the time it takes to separate two particles that are close to each other is much larger than the time the cluster formation takes to develop, thus creating transient clusters in the system. If the potential at the beginning is much smaller than the thermal energy, the system has time to develop clusters before the repulsive force has time to separate them similar to the transient cluster due to velocitydependent restitution coefficient [71].

$\Gamma^{-1}=7000$

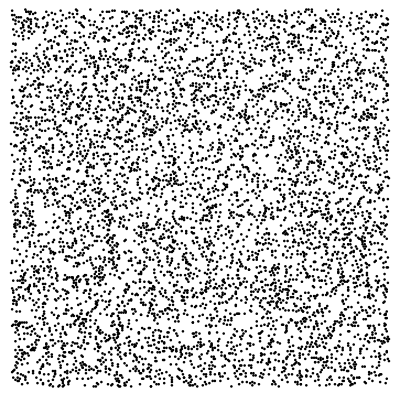

$\Gamma^{-1}=7.3$

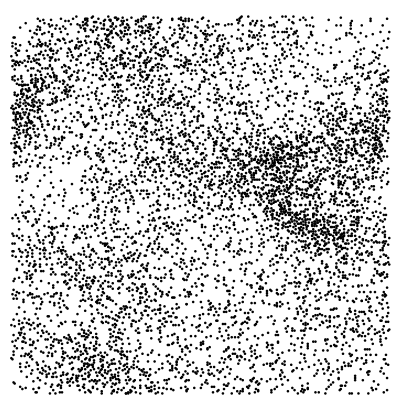

$\Gamma^{-1}=4.15$

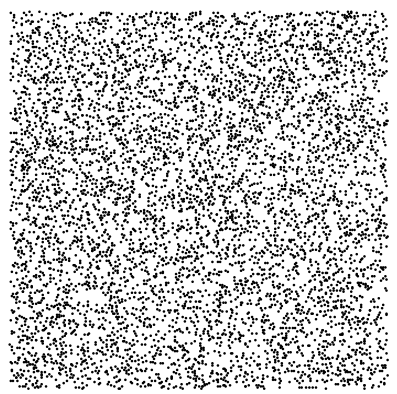

Figure 4.13: Simulation snapshots at different $\Gamma$ (time increases from left to right) for a weak repulsive potential $\left(\phi=10^{-4}\right)$ with $N=6400, \nu=0.057$ and $r=0.6$. In the picture of the middle the transient cluster formation can be seen; in this case, the kurtosis deviates towards a lower value due to the presence of clusters.

Figure 4.13 shows snapshots of the system at different times for a given repulsive potential and restitution coefficient. In the middle picture, the transient cluster structures can be seen, while before and after (left and right) the system is homogeneous. This corresponds to the evolution of the system represented in Fig. 4.14 by the dotted blue line.

Figure 4.14 shows the kurtosis of the velocity distribution as a function of $\Gamma$ for three different $\phi$ and restitution coefficient $r=0.99,0.95,0.9$. If the repulsive potential is weak enough and there is enough dissipation, the cluster instability can develop, making the distribution of velocities much 


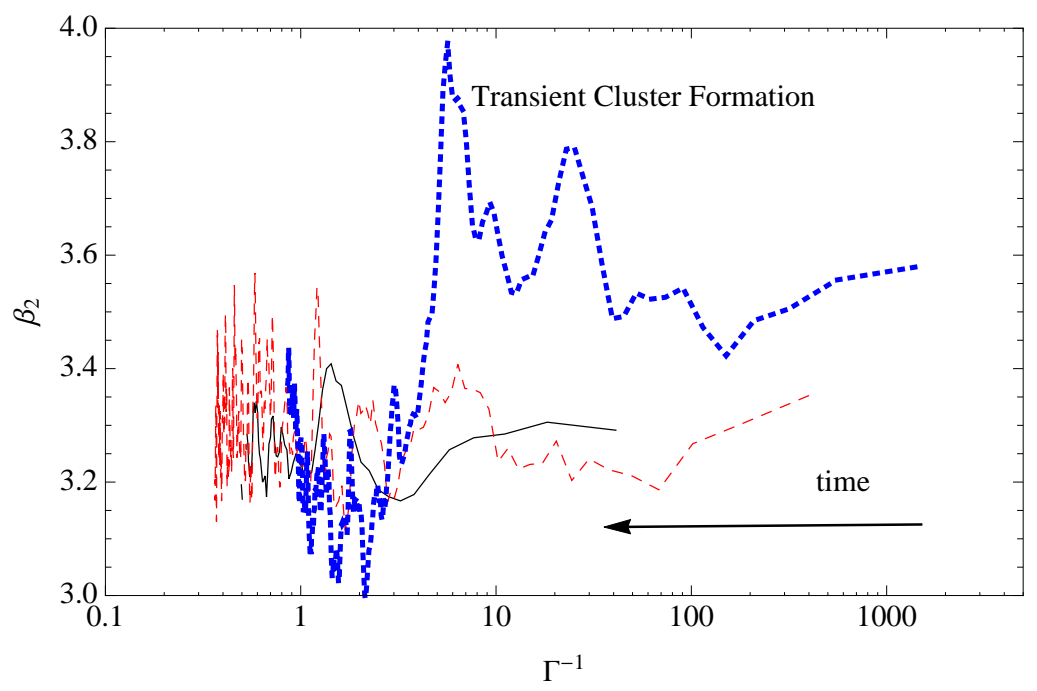

Figure 4.14: Kurtosis of the velocity distribution for three systems with $\phi=10^{-3}, 10^{-4}, 10^{-5}$ (solid, dashed and dotted lines) and different restitution coefficients $r=0.99,0.95,0.9$ as a function of $\Gamma$. The peak in the kurtosis at $\Gamma^{-1} \simeq 10$ corresponds to the appearance of a transient cluster. As $\Gamma$ approaches the unity, the velocity distribution tends to decorrelate and $\beta_{2}$ decreases.

more correlated and hence having a larger kurtosis (dotted line). When the thermal energy is comparable to $\phi$, i.e. $\Gamma \simeq 0.1$, the repulsive force starts to destroy the clusters and produces again a more Maxwellian distribution of the velocities. If the dissipation is small enough, the evolution for different coefficients of restitution in the plane $\Gamma-\beta_{2}$ is similar. The fluctuations around $\Gamma=1$ are due to the long time it takes the system to reach lower temperatures, and hence the evolution appears compressed when plotted as a function of $\Gamma$ instead of $\tau$. The fluctuations look larger than in the repulsive only because of the different scales of plotting; for large $\Gamma^{-1}$ both oscillate a similar amount.

\section{Transient formations}

The evolution of the free cooling with repulsive interactions is somewhat analogous to a system with a velocity dependent restitution coefficient, where cluster and shearing appear transiently [71]. However, the mechanism that determines the presence of clusters is the repulsive force being non-zero. Any amount of repulsive force between the particles will inhibit the cluster formation. Since there is no external pressure to compress the system, the particles always tend to repel each other. However, this repulsion mechanism has a temporal scale set by the intensity of the potential at contact. For two spheres touching, the time they need to separate a particle diameter, i.e. to 
do not feel the attractive potential, is

$$
t_{r} \simeq d / \sqrt{2 \phi / m}
$$

Consequently, unless the time of free flight for the particles becomes comparable to $t_{r}$, the repulsive potential is not able to separate the particles and the dynamics is dominated by the hard-sphere properties. Once the timescale of the system approaches $t_{r}$ the clusters begin to disintegrate and the system eventually returns to a homogeneous state.

\section{Effects of higher density}

For dilute enough systems the cooling rate becomes practically logarithmic, as is the case for a velocity-dependent coefficient of restitution. However, if the density is high enough, the system can reach an elastic regime due to the finite size of the system and the consequent bound for the kinetic energy. For such systems, the evolution is no longer independent of the coefficient of restitution, as it is the case in the dilute regime (data not shown). Since the barrier in the discontinuous case is abrupt, when the temperature is low enough particles cannot overcome the repulsive barrier and stop colliding. Then particles acquire an effective radius equal to the well width and the energy of the system remains constant.

Contrary to the attractive case, increasing the well width would be of no use in trying to recover the physics of the continuous potentials. What is needed in this case is to study how the inclusion of more steps in the potential approaches the continuous case. However, that is beyond the scope of this study.

\subsection{Phase diagram for cooling with long-range interactions}

Finally, the phenomenology for the clustering of hard spheres with long range interactions can be summarised as follows: for attractive potentials the free cooling systems always show clusters on the long term. If $\phi$ is large enough the cooling is homogeneous: once individual particles are bound together, the cooling continues as a cluster-cluster aggregation process. However, if the systems presents a shear or a cluster instability, the system will show inelastic clusters that cool down faster than the homogeneous hard-sphere case, giving rise to a different cluster structure.

For repulsive cases the homogeneous cooling is well predicted by a simple modification of the collision frequency. If the system is instable to clustering 
and $\phi$ small enough, the system will present inelastic transient clusters, in a way analogous to a granular gas with a velocity-dependent coefficient of restitution. Figure 4.15 shows a qualitative sketch of the phase diagram, for both attractive and repulsive potentials at a fixed density $\nu=0.057$ and different restitution coefficients and potential strengths.

A qualitative transition line can be drawn on the phase diagram by looking at how the critical restitution coefficient for the shear instability and a given system size changes as a function of $\phi$. From the stability analysis we have that for the smallest $k_{\text {min }}$, the critical restitution coefficient is given by $\mu_{0} k_{\min }^{2}=\epsilon_{c}^{2}$. If we add a long range potential the critical restitution coefficient will change. The transition line in the plane $r-\phi$ is given implicitly by $\epsilon^{2} \exp (\phi / T)=\epsilon_{c}^{2}$. Solving for this equation for the potential as a function of $r$, one gets in the repulsive case

$$
\phi_{c}=T \log \left(\frac{\left(1-r_{c}\right)^{2}}{(1-r)^{2}}\right)
$$

for $r<r_{c}$. In the attractive case, the critical $\phi$ reads:

$$
\phi_{c}=T \log \left(\frac{(-1+r)^{2}}{1+2(-2+r) r-\left(-2+r_{c}\right) r_{c}}\right) .
$$

In both cases, the critical line depends on temperature $T$. What temperature one should put there? If we use the initial temperature the line does not recover the behaviour we see for the repulsive case, where the system becomes stable for larger dissipation. If we use an arbitrary temperature of $T=10^{-3}$, the curve reproduces the trend form the simulations as seen in Fig. 4.15. Further study is required to understand the cause of this discrepancy.

\subsection{Conclusions}

The free cooling of granular matter with long-range interactions has been studied by means of event-driven simulations and stability analysis. The physical behaviour was investigated by means of stability analysis of the modified hydrodynamic equations where the dissipation rate was taken from a previous mean-field theory [72]. This theory was developed for 3D but we have shown that it also works for 2D systems in a straightforward manner. Simulations confirmed the theoretical prediction for low densities in the repulsive case (in $2 \mathrm{D}$ and $3 \mathrm{D}$ ) but felt short for the attractive case where non-linear effects are more important.

For attractive potentials the formation of structures, i.e., clusters, is strongly enhanced, as is expected from the results on condensation of elastic hard-spheres with attractive potentials [76]. This effect is not predicted 


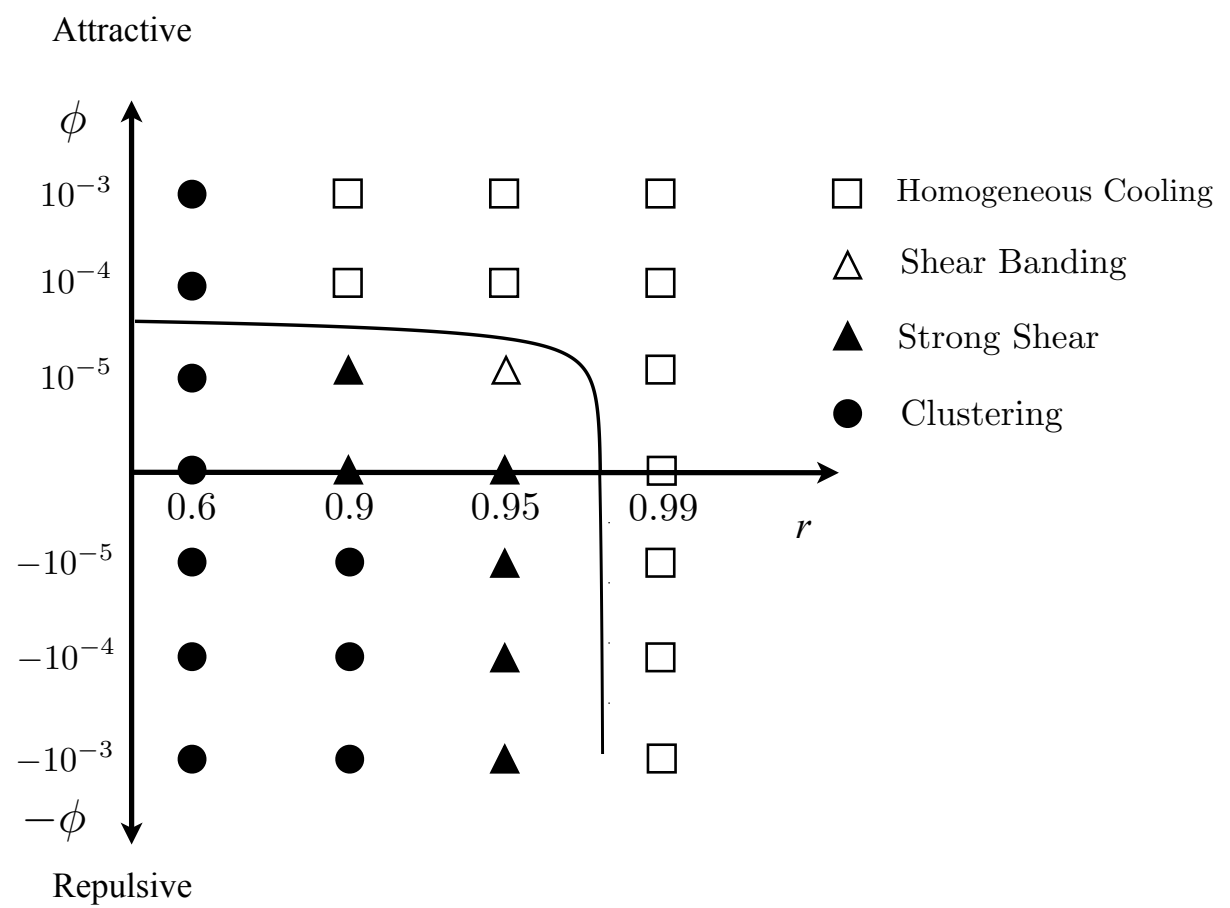

Figure 4.15: Phase diagram of the free cooling for the potential strengths $\phi$ and dissipation, $r$, for systems with $N=6400$ and $\nu=0.057$. The line is the transition curve for $T=10^{-3}$.

by the simple mean-field theory nor by the corresponding stability analysis where only the dissipation rate accounts for the long-range interactions. Indeed, the stability analysis of the hydrodynamic equations over-predicts the stability of the cooling with attractive forces; simulations show that the cooling is always instable to cluster formation, contrary to what the stability analysis says. Finally, the geometry of the resulting clusters is determined by a complex interplay between dissipation, potential strength and range, density.

For repulsive potentials, the theory predicts consistently the cooling behaviour for low dissipation and density when the system is homogeneous. This confirms once again that discrete potentials are a good approximation in this regime and capture much of the interesting physics of particulate systems. For larger dissipation and low repulsion we have found that transient clusters appear in the system in a way analogous to the cooling of grains with a velocity dependent coefficient of restitution. This is expected since the hydrodynamic equations have the same functional form.

Finally, these results point to the great importance of microscopic parameters in the macroscopic evolution of the system and the complex interaction 
of different phenomena. In particular, we showed that the long-time dynamics for a system with both dissipation and long-range interactions is not simply the addition of each effect but a complex interplay of forces that will depend on the evolution of the temperature from the initial state and the dissipation rate of the particles.

Future research in this subject comprises the unification of the algorithm from Chapter 3, together with long-range potentials. The idea is to be able to simulate the cluster of particles as solid structures, moving together, that can break and form in a dynamic way. This algorithm would make the simulations of dense and highly dissipative granular gases extremely fast, what could be applied, for example, to fluidised beds of nano-particles that agglomerate due to van der Waals forces [79]. 



\section{Chapter 5}

\section{Shaping Segregation*}

Controlling segregation is both a practical and a theoretical challenge. In this chapter we demonstrate that by introducing asymmetries along the axial direction of a rotating tumbler, the size-driven segregation in the axial direction of a granular mix can be controlled and speeded up. This observation and the new understanding that springs from its explanation - could lead to radical new designs for a vast set of particle processing applications.

\subsection{Introduction}

Granular flows in rotating drums present a rich variety of phenomena. They have been widely used to study mixing, segregation, and pattern formation [5]. While the majority of studies on rotating-drum flows focused on the circular cylinder geometry, several works, particularly in the last decade, explored similar phenomena in tumblers with other geometries [80-84]. Noncircular tumblers are important from both an application perspective, as they are used in various industries, and from a theoretical perspective, to further validate theoretical approaches developed primarily from observations of the simpler cylindrical configuration.

The role of geometry in mixing and segregation has long been known [85]. However, to the best of my knowledge this is the first study to present mixed convex-concave drum shapes. (Although there is some research on model SAG mills that present an indentation in the inner surface of the drum, hence weakly breaking the convexity, see e.g. [86,87].) As it shall be seen, concave rotating tumblers can be used to enhance the segregation of mixtures, a discovery whose applications could be far-reaching in industries ranging from pharmaceuticals to mining.

The structure of this chapter is as follows. I first introduce the system and explain both, experiments and simulations setups. Then, I characterise

\footnotetext{
*Based on S. González, S. Luding, A.R. Thornton, submitted to Phys. Rev. Lett..
} 
the dynamics of the granular flow in a concave tumbler. A comparison with the flow in a pentagonal tumbler, and in general with convex shapes is made and differences are highlighted. Bi-dispersity in size is added and I study the effect of different shapes in the segregation pattern along the axial direction. Finally, I demonstrate that the combination of different geometries along the axial direction can enhance the strength of segregation in binary mixtures along that direction.

The study is started with a drum shaped as a five-point star, commonly known as a pentagram. Its shape has puzzled humanity since the Sumerians; it was the symbol of perfection for the Pitagoreans; and, represented the five elements in ancient China. It belongs to the dihedral symmetry group and the ratio of its width to each side is the square of the golden ratio, 1.618..., and thus it was considered perfect by the Greeks. The first objective of this chapter is to explore the dynamics of a granular flow in a star-shaped tumbler as an example of a granular flow in a concave geometry. Since the star is not symmetric under reflexions on the horizontal plane, one expects different dynamics when the star is pointing up or down. The second objective is to explain such differences of the flow and harvest them to achieve a controlled axial segregation.

\subsection{Experimental set-up}

For the experiments, I use tumblers made of layers of $2 \mathrm{~mm}$ plywood laser-cut to arbitrary shapes. The drum is partially filled with glass beads and rotated at a constant speed $(\Omega=30 \mathrm{rpm})$ by a stepper motor. The granular medium is confined vertically between two acrylic plates in order to illuminate the bulk from behind and record it from the front (see Fig. 5.1, upper row). The length of the star is $a=119 \mathrm{~mm}$ and it is $W=10 \mathrm{~mm}$ wide, creating a relatively thin, quasi-2D configuration. Laser-cutting is a versatile and fast way to produce drums of arbitrary shape at low cost. Thus, one can easily perform studies that involve different shapes and optimise the shape for the required function. I use volume-coloured glass beads of nominal size $3.5 \pm 0.3$ $\mathrm{mm}$ and density of $2.5 \pm 0.02 \mathrm{~kg} / \mathrm{m}^{3}$ (manufacturer data sheet).

In the simulations, particles' contact forces are represented using a standard spring dash-pot model [14]. For the first simulations described here, slightly poly-disperse particles $\left(r_{\text {mean }}=3.5 \pm 0.03 \mathrm{~mm}\right.$, uniform distribution) are used and the same density as in the experiments. The boundary conditions are those of a horizontal rotating five-point star of length $a=119 \mathrm{~mm}$ and depth $W=10 \mathrm{~mm}$, periodic in the direction perpendicular to the plane of view in order to avoid boundary effects due to the walls. These are the same dimensions as the experiment. The rotation of the drum is achieved 

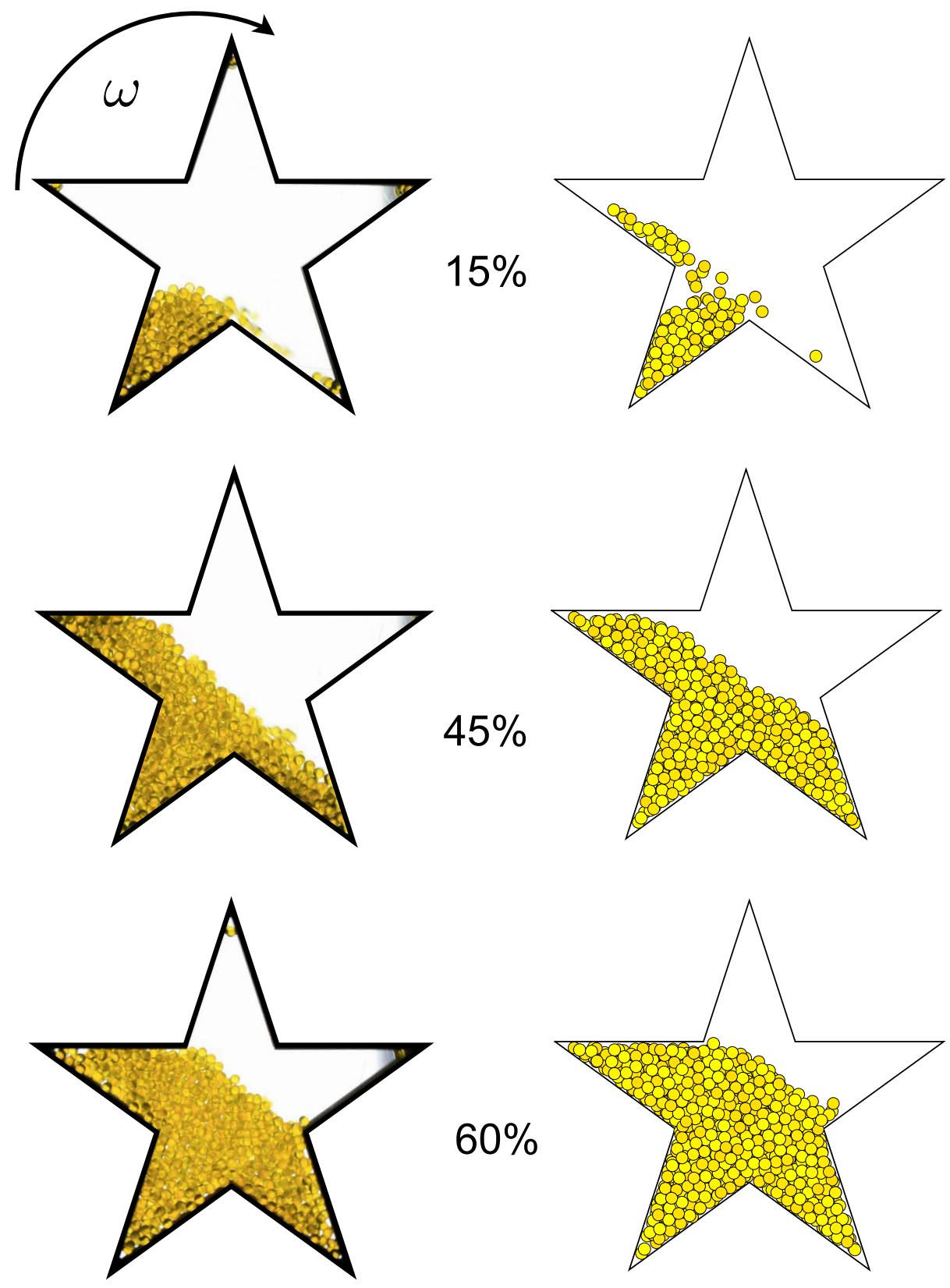

Figure 5.1: Granular flow in a rotating star: experiments (left) and simulations (right). The effect of increasing filling fraction (measured as the occupied area of the polygon) in the drum's dynamics can be seen from top to bottom $(10 \%, 30 \%$ and $60 \%)$. 
by changing the angle of gravity in the plane $\mathrm{x}-\mathrm{y}$ with an angular velocity of $\Omega=\pi / 2 \mathrm{rad} / \mathrm{s}$, hence mimicking the rotation of the walls. Since I am interested in the effect of the filling fraction, the angular velocity is fixed at this value since it provides a continuous free-surface avalanche [4].

For the bi-disperse experiments and simulations in the final section I use particles of 4 and $2.5 \mathrm{~mm}$, in a constant volume distribution, i.e. there is the same volume, and not number, of both particle types.

\subsection{The effect of the filling fraction}

There are four different regimes of flow depending on the filling fraction. If the grains occupy a volume smaller than that of one of leg of the star, the grains flow from leg to leg in an intermittent way, Fig. 5.1 (a). If the amount of grains increases such that there are always grains in at least two legs, the flow is constant but its angle changes due to the geometry, Fig. 5.1 (b). When the grains reach the level of filling between two and four legs, the flow is continuous but with roughly two different flow profiles depending on the angle of the star, Fig. 5.1 (c). Once the grains occupy more than four legs, the flow becomes intermittent and the displacement of the grains is strongly limited, so the transport of grains in the bulk decreases and the dynamics is mostly due to geometrical rearrangements (data not shown).

The most interesting regime corresponds to Fig. 5.1 (c). In this case one can clearly see the unsteady flow produced by a star-shaped rotating tumbler at constant rotation rate. The geometric shape naturally causes periodic changes in the flowing layer as a function of the instantaneous orientation of the star; something recently reported also in other geometries [84]. On this regime I focus next.

\subsection{Bi-stable flow}

In a cylindrical rotating drum, the steady flow has a constant kinetic energy. For the star, however, the flow is oscillating: when a star tip is pointing up, the flow is slow while when the star is pointing down, the flow is much faster. Figure 5.2 shows the instantaneous velocity field for both the star and the pentagon. The star shows a great variation in the magnitude of the velocity between the pointing up versus pointing down configuration. Whereas the pentagon shows a more constant flow pattern; in both orientations the magnitude of the velocity is similar, and only the length of the flowing layer is changing.

The explanation is that when the star is pointing up, the avalanche occurs in a thick layer of particles. As the star rotates, more space becomes 
available for the particles to flow and there is a saltation flow going on. This produces a fast avalanche in the down part of the flow, and the consequent movement of all the flowing layer. In turn, this explains why the total kinetic energy of the particles shows five maxima during one cycle, see Fig. 5.3. By allowing particles more space to flow, a big, fast, avalanche is produced. This avalanche is not symmetric along the free-surface. Most of the kinetic energy is on the downside, where it is easier to flow due to the free volume. Eventually, the leg of the star is filled with particles and the avalanche recovers its slow flow. As the star turns the process repeats. Now I want to focus on how this feature can be used to control segregation. To do this, one needs to introduce the convex counterpart of the star, the pentagon.

\subsection{Comparison of Star with Pentagon}

The flow in a concave tumbler is clearly different from the flow encountered on convex tumblers [80-83,88]. Take, for example, a rotating pentagon that has the same rotation symmetry as the pentagram but is convex. The differences in their respective flows should be due then only to the concavity/convexity of the tumbler.

As the pentagon rotates, the total length of the flowing layer changes, hence creating an oscillation in the kinetic energy with the same period as the pentagram, see Fig. 5.3. However, this flow is much more continuous in the pentagon and the velocity of the flowing layer is more constant and its variation between minimum and maximum is smaller.

The periodic structure of the kinetic energy (see Fig. 5.3) can be understood by simple arguments. If one considers the speed of the flowing layer and its depth constant, then, the kinetic energy is proportional to the length $L$ of the flowing layer. Disregarding the angle of the walls (i.e. assuming a square instead of a pentagon so I disregard the fact that the walls in the pentagon are not parallel), the length of the flowing layer scales as $L \propto 1 / \cos (\theta)$, with $\theta \in[0,2 \pi / 5]$ the angle of rotation modulo the symmetry of the shape, in this case $2 \pi / 5$; see fig. 5.4 for the explanation (and Ref. [84] for an example in a triangular geometry). Hence, the kinetic energy is $E \propto 1 / \cos (\theta)^{2}$. The agreement of the simulation with this simple model is remarkably good, as can be seen in Fig. 5.3 by the agreement between the solid and dotted line.

The explanation for the such a behaviour of the kinetic energy is the following. Consider the mean velocity of the system written as a continuous field with the free surface in the horizontal and assuming a constant velocity 
in $z$, for simplicity,

$$
\bar{v}=\frac{1}{A} \int_{-L / 2}^{L / 2} \int_{-L / 2}^{H} v(x, y) d x d y
$$

where $A$ is the total area of the system. Since the packing fraction remains roughly constant, so does the area. One can make a change of variable in the $x$ direction,

$$
\bar{v}=\frac{L}{A} \int_{0}^{1} \int_{-L / 2}^{H} v\left(x^{\prime}, y\right) d x^{\prime} d y .
$$

Using, for example, the velocity profile proposed in Ref. [89], the integral is a constant. Since the flow depth is a fixed height, $h$, much smaller than the vertical size of the flow, the integral is independent of the angle of rotation of the tumbler. So, for an arbitrary angle, one has that the average velocity

$$
\bar{v}(\theta) \propto L(\theta)
$$

In a pentagon, the flowing layer is at the edge of the geometric region of constant volume. This region is always connected for convex shapes and thus the filling height $H$ does not change much. However, for concave shapes the constant-volume regions are not always connected. This causes $H$ to change strongly and discontinuously as the star rotates. Consequently, both of the last assumptions, namely that the flowing layer is smaller than the material height and that the vertical profile is independent of the angle of rotation, are broken for concave shapes. In this way, grains flow faster when they encounter a "run-out" leg with free volume, where the relative change in the container's wall angle affects the velocity of the flow.

This fundamental difference between the flow in a convex and concave rotating tumbler - in the concave case there is a run-out leg with faster flow - is the key ingredient to develop a new mechanism of segregation, as shown in the following. 

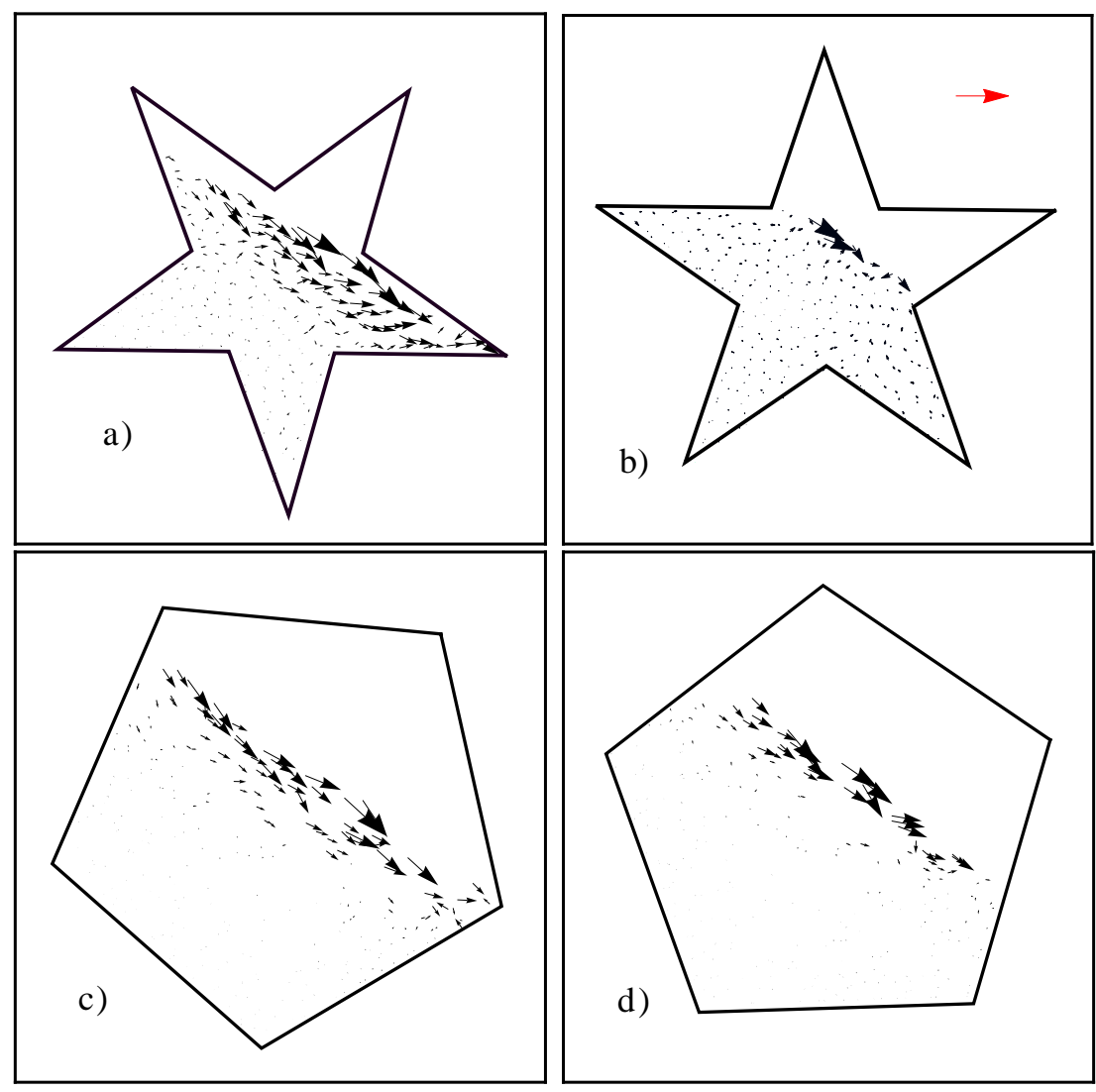

Figure 5.2: Velocity field for two snapshots of the simulation on the moving frame and a filling fraction of $60 \%$. The arrows represent the instantaneous velocity in the $\mathrm{x}-\mathrm{y}$ plane for all the particles. In all the pictures the scale is the same; the red horizontal arrow representing $0.25 \mathrm{~ms}^{-1}$. For the star pointing up (b) the flow presents a typical rotating drum profile that decreases exponentially from the free surface. When the star is pointing down (a), the right bottom leg provides the space for a shallow and fast avalanche to occur. For the pentagon, the energy per particle in the avalanche remains roughly the same (c and d), but with more particles flowing when the pentagon is pointing down (c) since due to the geometry, there are more particles available to flow. 


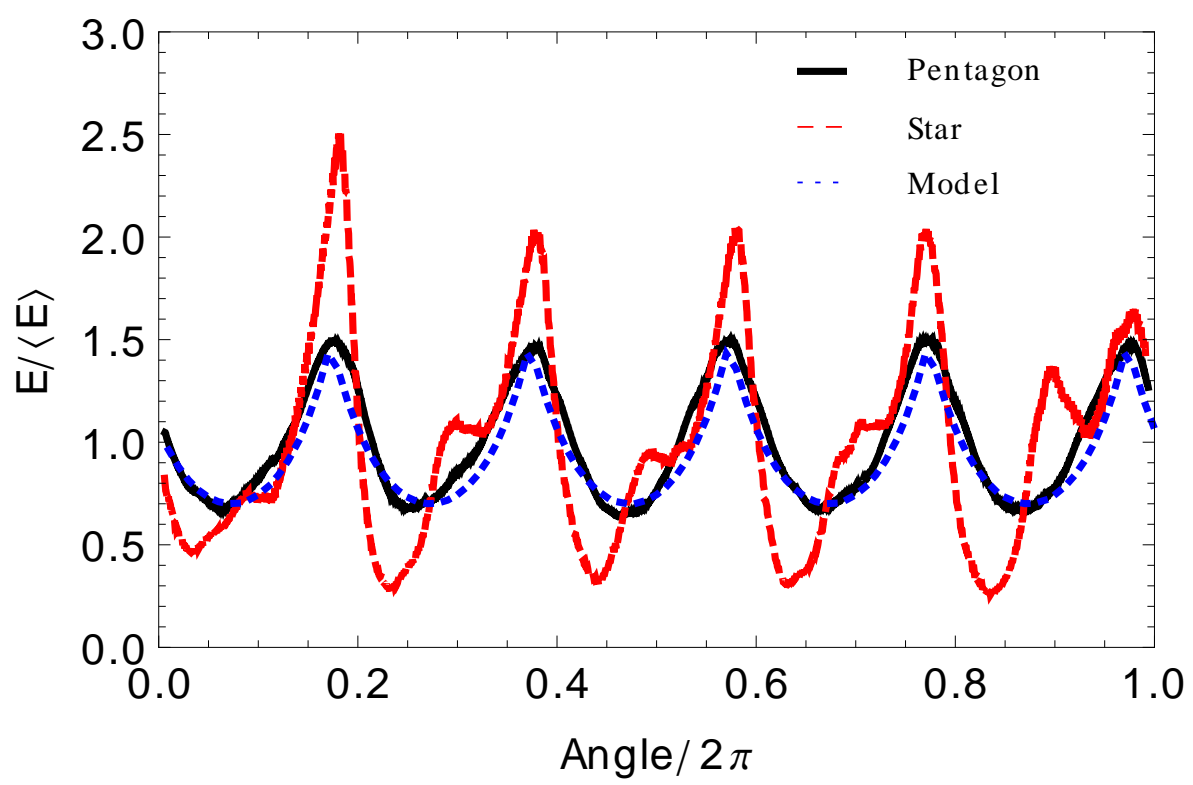

Figure 5.3: Kinetic energy as a function of time during one cycle for the simulation of a pentagonal tumbler (solid, black), a star (red, dashed), and the result form the model for the pentagon (blue, dotted). The structure and angle of rotation of the drum can be guessed from the energy evolution. In this case, both systems show the same temporal structure of five peaks per turn. However, the star presents a relatively faster avalanche when pointing down; this is the main feature that it will be used in order to induce segregation along the axial direction. Data averaged over eight cycles over one simulation of each geometry. 


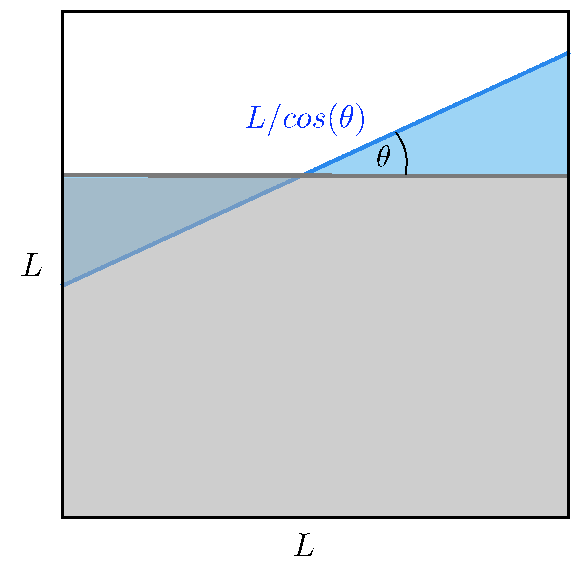

Figure 5.4: Relation of the length of the free surface to the rotation angle for the case of a square tumbler. Since the occupied area is conserved as the square rotates, the length of the free surface is determined by the geometry. This formula is valid in $\pi / 2$ intervals. In the case of the pentagon, the analytic formula is more complex, but as a first order approximation one can consider the same functional form as for the square only valid in $2 \pi / 5$ intervals. 


\subsection{Shape-induced axial segregation}

The differences in the flow patterns presented by a star and a pentagon can be used to influence the segregation behaviour of a bi-disperse mixture. The influence of the container's shape on segregation patterns has already been reported [90,91], however, this is the first time that it is used in a rotating tumbler. It is also known that modifying the geometry (e.g. by adding obstacles or mixing blades in the drum) can reduce segregation [92] but it has not been shown how to improve and control it - a matter of obvious practical importance.

When using a bi-disperse mixture of particles in any rotating container, the small particles will go preferably to the centre of the drum irrespective of the drum's shape $[5,93]$. This is also the case for the star and the pentagon: small particles are preferably in the centre of the drum, see Fig. 5.7.

However, if two different geometries are used along the axial direction, say, by making a tumbler half star-shaped and half a pentagon along the axial direction, the usually slow segregation in this axis can be enhanced and its direction controlled, see Fig. 5.7. In both experiments and simulations two sections with the same width of the original experiment are combined, hence doubling the size of the drum along the z-axis. The rapid axial segregation happens only when a convex and non-convex shape are brought together, as for convex shapes the flow does not show much differences in the level of the flow, just its length. I performed different experiments putting together circular and square sections, pentagonal and square, and square sections rotated in different angles (data not shown). None of these configurations presented axial segregation on the time scale of observation (around 20 revolutions).

One can understand that the change in the geometry - from convex to concave - of the drum creates a level difference between the two sides of the tumbler due to the strong variations on the concave side. The grains tend to go to the side of less potential energy, i.e. the pentagonal side, which happens to be also the side with more free-volume. This can be seen from Fig. 5.5: the segregation occurs in an oscillating way, when the big particles drop from the run-out leg of the star to the pentagon the centre of mass of the large particles shifts to the negative side (and the one of the small particles to the positive sides). However, since there is mixing in the system some large particles go again to the star side. In this way, an oscillating movement of the centre of mass of each species is observed: big particles fall to the pentagonal side five times per turn when the run-out leg is empty; once the flows covers the run-out leg some large particles return to the start side. The drift of particles follows a clear trend until it saturates around the eighth revolution.

As the mix is bi-disperse, in each side the radial segregation is taking place 


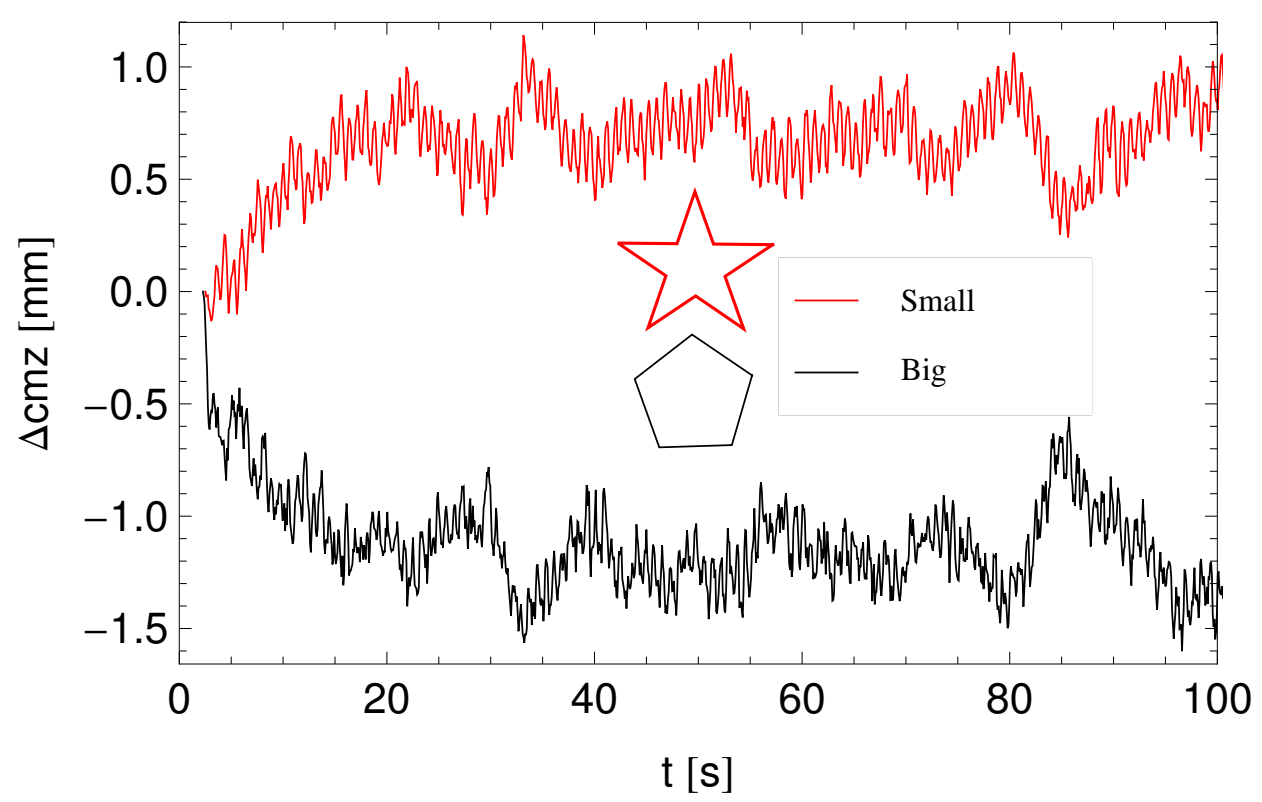

Figure 5.5: Evolution of the displacement of the centre of mass for the simulations at $50 \%$ filling fraction in a star-pentagon geometry, each of width $\Delta z=11 \mathrm{~mm}$. Small particles move towards the star side $(z>0)$ while the big particles move to the pentagonal side $(z<0)$. The system equilibrates after some 8 turns $(32 \mathrm{~s})$.

so the large particles go to the surface and the small to the centre. Since for this packing fraction the avalanche in the pentagonal side of the drum $(z<0$ in the simulation domain) is slower than in the star-shaped section $(z>0)$, the large particles can move to the empty side since they are faster and there is room for them in the pentagonal side. Once the two avalanches reach the same angle there is no more flux of particles. This process is repeated five times per turn. By this mechanism, there is a net transport of large particles to the convex side of the drum while the non-convex side remains mostly composed of small particles. This mechanism is orders of magnitude faster than the phenomenon of banding previously reported for granular materials in axially homogeneous tumblers [94].

One must note that the segregation is not the same for every filling fraction. Figure 5.8 shows the change in the number of large particles in the pentagonal side of the tumbler for different filling fractions. If the system is too empty, the avalanche on the star side of the drum arrives at the same time than the one in the pentagon and the axial segregation is slower. It must be noted that there is also axial segregation due to the wall; large particles tend to be close to walls while small particles are mostly in the centre, in accordance with [95].

This points to the existence of two mechanisms of segregation. On the 

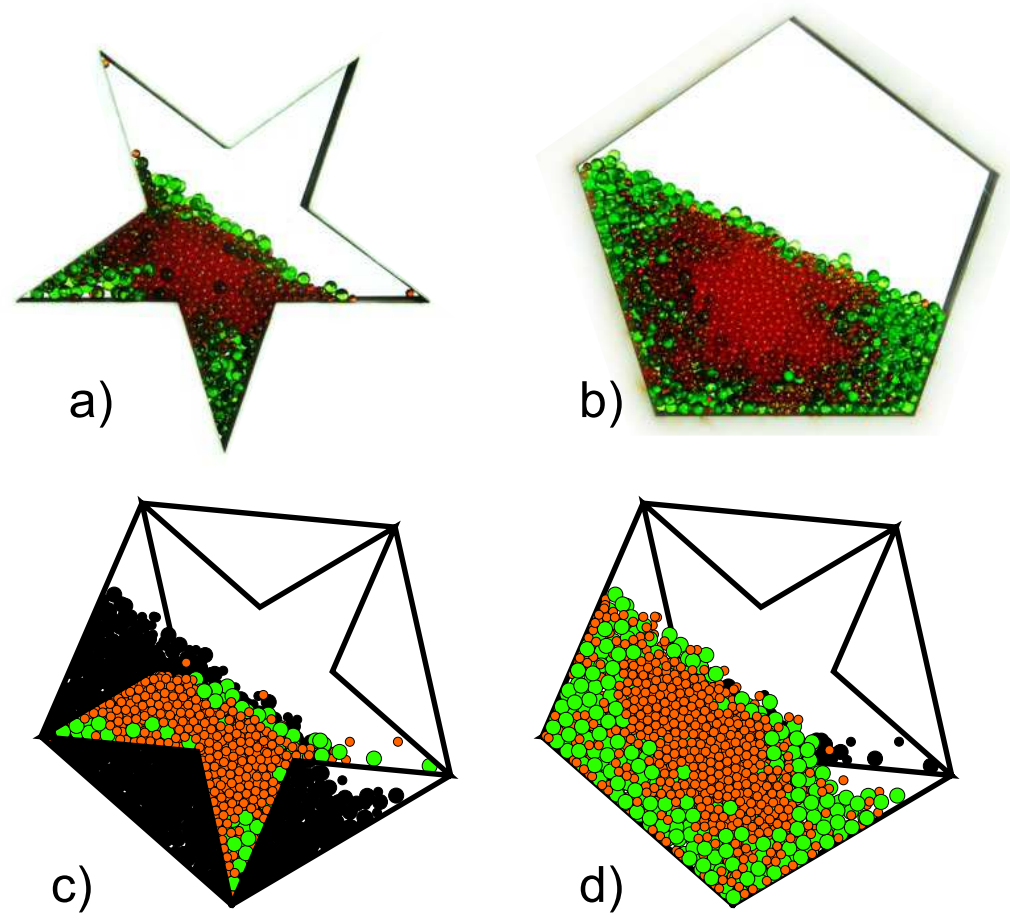

Figure 5.6: Photographs of the experiment after four revolutions in the axially homogeneous tumbler for the star (a) and the pentagon (b). Simulations for the axially inhomogeneous, layered tumbler after four revolutions, from the front (c), i.e., the star-shaped side, and from the back (d), the pentagonal side. Particles are coloured by size with orange small and green large; black particles correspond to those particles that belong to the opposite side of the tumbler. It can be seen in (d) that the particles in the back, run-out zone (black particles) are higher than those in front (green and orange), and consequently, they can eventually fall to the pentagonal side.

one hand, the excluded volume effects make the small particles to go preferably to the star side since the large particles do not fit on the legs so easily, in accordance with [91]. However, this mechanism does not explain the maximum on the segregation at around $50 \%$ filling fraction. This is can only be due to the flow structure as previously discussed.

\subsection{Conclusions}

In this chapter I showed that for a five-point-star shaped drum filled with grains there is a rich flow behaviour, depending on the filling fraction due to the non-convexity of the drum. Different regimes are found for a fixed angular velocity. From intermittent avalanching (low filling fraction) to geometrical rearrangements (high filling fraction) passing by continuous flow 
(around half filled). By controlling the shape of the drum, it is possible to produce different dynamics with a single driving frequency; if different dynamic regimes coexist in a drum along the axial direction, segregation will occur. I have used this insight to control the segregation pattern in a binary mixture of grains, achieving fast shape-induced size segregation along the axial direction. The possible applications of this mechanism can be revolutionary. Firstly, this is a way to produce axial segregation orders of magnitude faster than previously reported. Secondly, this can be used, for example, on rotating kilns to have differential residence times depending on the size of the particles. Finally, this mechanism can be used on milling devices: by doing a sandwich of concave sections with a convex shape in the middle, large particles can be induced to go to the middle of the tumbler, thus making the milling more effective by keeping the grinders and larger particles in the mill while moving the fines to the sides, where they can be removed. The range of application of this discovery goes from the production of pharmaceutical products to improved mill design in the mining industry and sheds new light onto the role of the boundary condition in segregation problems.

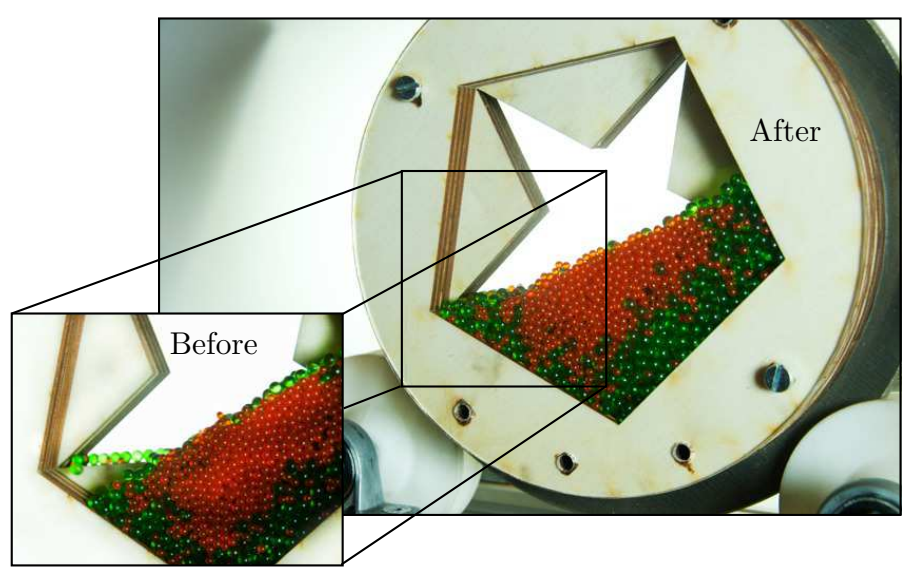

Figure 5.7: Photographs of the experiments showing the mechanism of segregation, before and after the flow in the pentagon catches up with the on in the star. On the star side (before), particles have a larger potential energy and thus tend to flow to the other side. As the tumbler rotates the run-out zone is filled with particles (after). 


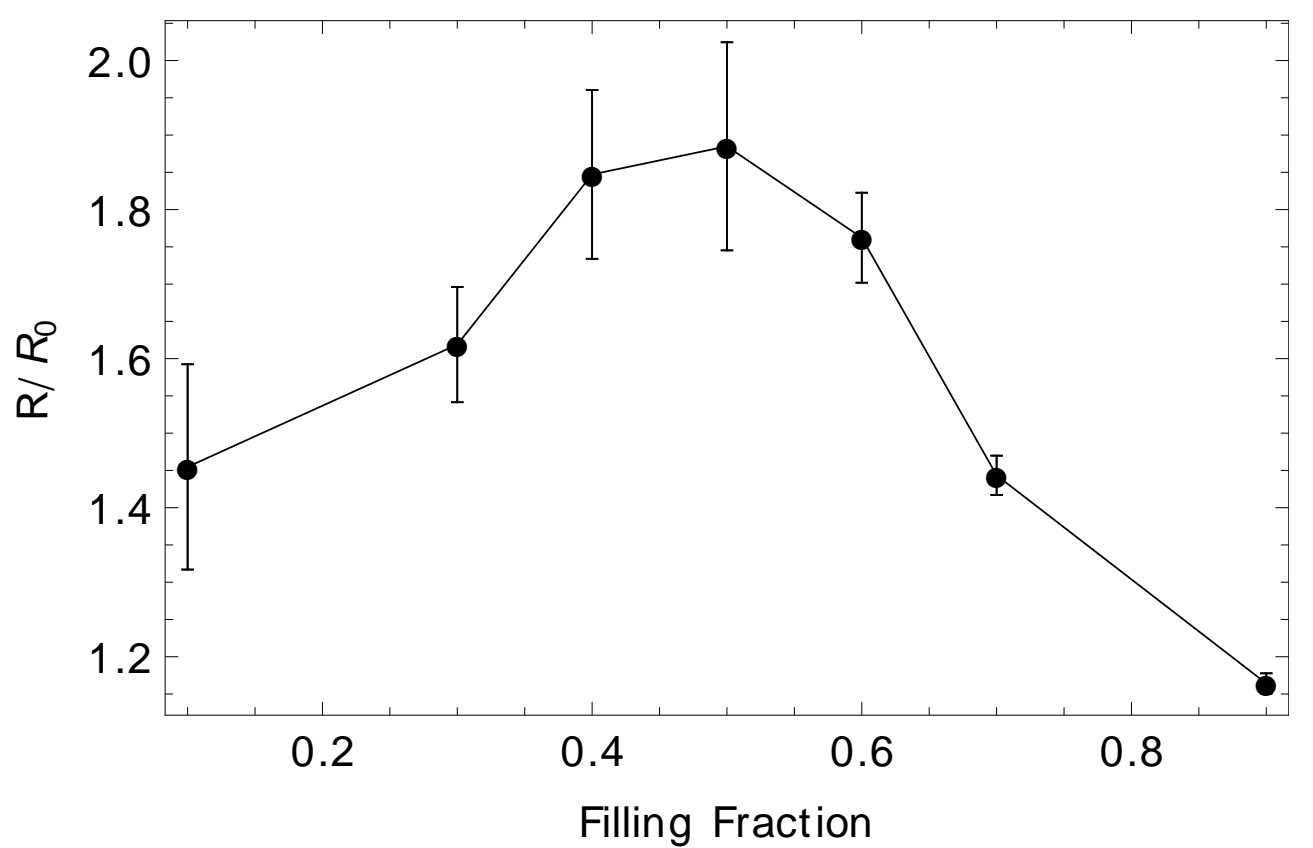

Figure 5.8: Number ratio $\left(R=N_{\text {large }} / N_{\text {small }}\right)$ in the pentagonal side of the drum $(z<0)$ versus the filling fraction normalised by the initial conditions. A value of two means that the ratio of large to small particles is twice the initial value. The segregation has a clear maximum for the half filled configuration, however, it also has a larger error. Data is averaged in ten snapshots during two turns of the tumbler and the errors bar are the standard deviation of these measurements. 


\section{Chapter 6}

\section{A ritual that is not rotten*}

Tout problème humain demande à être considéré à partir du temps. L'idéal étant que toujours le présent serve à construire l'avenir.

Franz Fanon

\subsection{Introduction}

In April 2011, Nature published a special edition covering the future of the $\mathrm{PhD}{ }^{1}$. In it, different authors argued that the PhD must be completely reformed: too many $\mathrm{PhDs}$ for not enough University positions; a formation that is too specialized to be used in industry; no noticeable economical gain for PhD holders compared to Master graduates.

On top of this less than exciting diagnostic for the $\mathrm{PhD}$, Science nowadays is more and more mistaken for an economical endeavor ${ }^{2}$; the publishing model favors the profit making of private companies instead of the communication of science; research founding promotes the repetition of well proven recipes instead of adventures in unknown lands.

With such a perspective I felt that my job was utterly senseless and pointless. However, I deeply believe that science is something worth giving your life for, something that changes the world, and that it is beautiful.

Trying to resolve such a deadlock, I came to face the question "What is Science?" There is no easy answer. Furthermore, I was obliged to take a philosophical position in order to attempt to answer this question. This philosophical study then became both a vital and an academic task for me.

*A shorter version of this text was presented at the music festival Green Vibrations, 17th of May, 2012, Enschede, The Netherlands.

${ }^{1}$ Available at http://www . nature.com/news/specials/phdfuture/index.html

${ }^{2}$ See Nature Volume 484 Number 7392 (2012). 
In what follows I introduce the philosophical perspective I chose to tackle this problem and some of the conclusions I have reached.

\subsection{The Beginnings}

\section{The Event of Science}

A long long time ago, in a faraway land, two men are talking. Maybe Socrates and Parmenides; however, their names are unimportant. Two Greek men talk about abstract matters. They argue, but they are not interested in winning or losing.

They know something: discussion is the not-impossible path to arrive at the truth. They refused to accept any pre-given opinion, any tradition without questioning it, and so they look for the Truth in itself. They have forgotten praying and myths.

The attitude of these two unknown men toward their world rapidly grew into a defined cultural form that the Greeks called philosophy. This was nothing but universal science, science of the world as a whole, of the universal unity of all being.

Very soon, the interest in the totality and, by the same token, the question regarding the all-embracing becoming - Heraclitus' river - and the resulting being - the being with capital B from the philosophers - began to particularize into disciplines. So science began. ${ }^{3}$

\section{My path to Science}

The house surrounded by shit. That is the name I have for Latin-America. It comes from a poem by Nicanor Parra, a fellow Chilean. The poem is called Flies in the shit and goes like this:

To the gentlemen - to the tourist - to the revolutionary

I would like to ask them just one question:

have you ever seen a fleet of flies

hover around a piece of shit

land and work in the shit?

have you ever seen flies in the shit?

because I was born and raised with the flies

in a house surrounded by shit. ${ }^{4}$

\footnotetext{
${ }^{3}$ This account is based on Borges' poem El origen and Husserl's Philosophy and the Crisis of European Man.

${ }^{4}$ Nicanor Parra, Emergency Poems (New York: New Directions 1972), our translation.
} 
I was born there. In the house at my right, a step-father raping and impregnating his 15-years-old step-daughter. In the house at my left, an 80 years old woman getting older and loosing her mind, living alone, getting her purse in the middle of the night to go to the dentist, only to fall right outside her door, crying. I was trying to convince her to go back to bed, telling her that it is midnight and it was cold. I was twelve by then. In my house... well, a bit of violence, not so much - a bit of poverty, neither that much for Latin American standards. A house with books at least. With an encyclopedia whose pages I read over and over again, realizing that there were truths. No matter whether I woke up to the sound of breaking glass in my parents bedroom, there were truths. There is something that is there and does not ask you where you are from, what is your sex, and how you look to be learned. It is there, it is free, it is yours: Knowledge. And I decided that I wanted to understand. Understand the world.

I thought in my childish mind that if you were able to understand the world, the human suffering I saw around me could be understood and hence changed.

Eventually I grew up, I studied physics in the best university of Chile and managed to escape and live in Europe where I'm finishing a PhD.

After a year in Europe, I came back to Chile for a conference and was invited to visit the University of Chicago. I went there for two months. It's the best place in the world to make experimental granular dynamics, my subject of research. The same university responsible for the atomic bomb ${ }^{5}$ and the neo-liberal economic policies ${ }^{6}$.

I went there with my bike and my pink comforter. After a week or so I wanted to kill myself: for my life as a scientist to go undisturbed, I needed the police cars to protect the closed environment of the University of Chicago so the black people from the ghettos around the university would not come in to kill us. ${ }^{7}$ And then science appeared to me as not worth it. No discovery would make the situation right, no new idea, no new invention could justify science if science doesn't care about the world.

I was depressed in Chicago. I was sad. I felt utterly alone and a feeling of senselessness invaded me. The only thing I could think was: it is not worth it, science is not worth it.

And nobody seemed to care about it. In the building I was, some of the most intelligent men of the world; a few blocks south, black people living in poverty killing each other. It was natural for them. That was the way things were.

\footnotetext{
${ }^{5}$ See http://en.wikipedia.org/wiki/Chicago_Pile-1

${ }^{6}$ See http://en.wikipedia.org/wiki/Chicago_boys

${ }^{7}$ For some data on Chicago's distribution of poverty, see http://www.city-data.com/ poverty/poverty-Chicago-Illinois.html
} 


\section{The deadlock of doing science}

So after 10 years of study, I was faced with the same problem that I was faced with as a child. There is suffering in the world and the method I chose to explain reality was not able to say anything about what really interested me. The feeling, the certitude that the world was wrong could not be grounded in any scientific statement. It could not be demonstrated as I used to do with my physics problems. My naïve idea was that if one could demonstrate that the way society works is wrong, people would understand and change their ways. Now I see that this is not possible nor desirable. Furthermore, having access to the whole truth implies the end of freedom, and to take a decision with all the knowledge means no courage at all. ${ }^{8}$

Then my question was: how come this conversation the two Greek men had so long ago became this? Why did science take for granted a state of the world as if it were something natural? Why did we betray our forefathers? I have spent the last two years trying to understand this, and the more I got into it, the more it seems to correspond to a state of the world and not only to science.

Today, science and technological innovation are necessary for the material working of capitalism. Does scientific knowledge also imply a theoretical support of the economic and politic order of the world? Or on the contrary, is there an idea of a better world beyond what we know today that comes from natural historical necessity, as the japanese philosopher Kojin Karatani sustains [96]? Or none of the above but, worse, is it the case that science cannot talk about politics due to a constitutive and insurmountable lack of knowledge, and should remain silent there where it cannot speak?

To answer these questions we need to make a genealogy of Science.

\footnotetext{
${ }^{8}$ This is Kantian insight in the Critique of Practical Reason. If we were to gain access to the noumenal domain, to the Ding an sich: "...instead of the conflict which now the moral disposition has to wage with inclinations and in which, after some defeats, moral strength of mind may be gradually won, God and eternity in their awful majesty would stand unceasingly before our eyes. [...] Thus most actions conforming to the law would be done from fear, few would be done from hope, none from duty. The moral worth of actions, on which alone the worth of the person and even of the world depends in the eyes of supreme wisdom, would not exist at all. The conduct of man, so long as his nature remained as it is now, would be changed into mere mechanism, where, as in a puppet show, everything would gesticulate well but no life would be found in the figures." (Immanuel Kant, Critique of Practical Reason, New York: Macmillan 1956, p. 152-153.) So, for Kant, the direct access to the noumenal domain would deprive us of the very "spontaneity" which forms the kernel of freedom: it would turn us into lifeless automaton, or, to put it in today's terms, into "rational agents". The basic gesture of Kant's transcendental turn is thus to invert the obstacle into a positive condition. To turn the limits of our cognition into the condition of our freedom. The impossibility of knowing all becomes the condition of possibility for our doing freely.
} 


\subsection{Genealogy of Science}

Summarizing greatly, the conversation that the Greeks started, passed to the Romans and when Christianism appeared it was "baptized". This lasted a good thousands years. So in the middle ages, universities where under the church dominance. Knowledge was only passed through, teaching Aristotle. (Remember Aquinas' "creatura non potest creare", creatures cannot create.) There was a clear understanding of a flat and ordered world. Quite sound and beautiful, but utterly false.

In the 1600s one of the greatest revolutions of humanity occured. A man, Galileo Galilei showed that the worldview the church was putting forward was simply wrong. Thanks to him, we got rid of Aristotle, the Church lost its central place in the world, and we proved that reason was the way to understand reality.

This new vision of the world that appeared with the scientific revolution of Galileo, and the consequent changes in social structure (formation of nations, state and a capitalist economic system), transformed the role of universities and included for the first time the creation of knowledge as a mayor point. This was the German model of universities, developed by Alexander von Humboldt. In 1810, Humboldt convinced the King of Prussia to build a university in Berlin based on Friedrich Schleiermacher's liberal ideas; the goal was to demonstrate the process of the discovery of knowledge and to teach students to "take account of fundamental laws of science in all their thinking." Thus, seminars and laboratories started to evolve. In this way the student becomes a researcher and the teacher a guide.

In the nineteenth century and the beginning of the twentieth, research was carried out mostly in universities. There was however, a significant amount done also in institutes, private gardens and houses, or even patent offices, using well-thought-through communication with low frequency, in contrast to today's practices. Time passed, there was a world war, then another, and scientists moved from one continent to the other, where they ended up working on atomic bombs among other things.

This was an important shift since transformed science - that was a national endeavor, with journals in national languages - into a global enterprise with its common language, English.

After the second world war the economic dominance of the United States imposed an economic worldview that supposedly will bring freedom to the world. The invisible hand of the market knows what's best for the world and America will take care to impose the market on all the world and in every sphere of existence, from the commodification of sex to the patenting of genetic code and living beings. 


\subsection{The University Today}

In what follows, I want to focus on the economisist worldview that has permeated science, endangering its practice. I start with an example: a friend of mine received a letter from the University of Twente that addresses him as "Beste Klant...", and continues to inform him that if he doesn't pay his debt of 3.000 euros, he won't be able to continue his studies. Signed by Credit Department, UT.

This is something that can only happen at the beginning of the 21st century. Education is not seen anymore as a right or a privilege but as an economic relation - an investment by part of the consumers who acquire a certain good, namely education, that will eventually result in profit for the consumer. In this view, the University becomes a business, and science, the Trojan horse that will convince costumers to apply to one or another university. Hence, the dominant discourse becomes one of efficiency, productivity and valorization.

Historically, this world view extended worldwide with the fall of communist Russia and the imposition of capitalism in China. There is no alternative - a slogan made infamous by Margaret Thatcher - history seemed to be saying to us, this is the best you will ever get, or at least the less bad. Only in such a worldview a student can be called a "client", a label that for me is outrageous and contradicts everything that education and culture stand for.

In science, this translated in the need to "measure" scientific output. Since the easiest thing to measure is the number of publications - computers are really good at doing that - the means of communication, i.e. the paper, became the end of science. What matters today is, mostly, not to find truths but to publish papers. Of course there are still exceptions, and good science continues to be made, but when we publish more than a million papers per year, something tells me that we are doing something wrong.

\subsection{What science can say}

As a scientist, one should attack this economic view for several reasons. First, the validity of its axioms is highly questionable from a scientific point of view. Second, the economist view is sensless in scientific context, as I will argue later. Third, the economic developement nowadays is putting our biological support in a strain hitherto unheard-of, endangering our own survival as a species. ${ }^{9}$

\footnotetext{
${ }^{9}$ I think the perfect counter example of what I propose here is the anti religious prattle of Dawkings and Dennet, amonsgt others. What we have today, instead of a proper political intervention from scientists, is intolerant racist propaganda veiled as atheism. What Dennet and Dawkins do, is criticizing religion because of its content, while formally
} 
What are the axioms of economic practice? The invisible hand of the market, rational agents and exponential growth.

Think of you, do you really think you are rational? Have you ever been in love? The truth is that we are not rational, at least not all the time. That we do things for different reasons, not only calculating which is the best output for us. This is a biological fact. The truth is that we do not know what human nature is. We are not our genes nor our synapses, as some reductionist authors pretend. Science is no closer to understand what the human is than Borges was when he said that we are drops in Heraclitus' river.

The invisible "hand" of the market in turn is a metaphor with no explicative value, it explains nothing. Furthermore, there is no such a thing as free markets, nor markets make free, as in freedom. They are always already inside a political frame. Why can't I buy kids? or human kidneys? or love? Because not everything is a commodity nor everything should be a commodity.

Economic growth is another such dogma: "To believe in unlimited growth right now still seems to be an act of pure faith" ${ }^{10}$ [97]. We live in a finite world, and if alone for thermodynamic arguments ${ }^{11}$, we cannot continue growing indefinitely.

In the next section I attempt a different approach to understand science, one that seems to me to contain more truth. For this, we need to make a detour through philosophy.

\subsection{Philosophical Position}

To talk about science always presupposes a definite philosophical position. There is no neutral point from where to look at it. Mark Taylor in an editorial

behaving as the same religious people they attack. In their critic, they assume that occidental institutions are the natural result of evolution, and hence they justify the state of the world as something necessary, being religious de facto. Science did not undermine religious power because of a particular animosity regarding religion. It undermined religion because the institution of religion was based on precepts that contradicted the material grounding of the world. If we want a scientific critic of society, today it should start by capitalism, for is the where most of our dangers come from (global warming, food sovereignity, dna-manipulation, etc.). What are a few Islamic terrorist compared with the harm a copyright law on seeds brings to the world? Science's depoliticizing is not its greatest achievement but the mere price of its survival in today's world. I see no reason for not taking science's premises to their logical conclusion, even if this means that by doing so science becomes politicized.

${ }^{10}$ This article, appeared in Nature a good six months after I gave my talk, gives me hope on science: we are finally saying (in a high impact journal, the epitome of the scientific establishment) what has been in front of our eyes for years, and we cannot continue pretending it is not there.

${ }^{11}$ http://physics.ucsd.edu/do-the-math/2012/04/economist-meets-physicist/. 
comment in Nature ${ }^{12}$, for example, talks about the "global competition for the multibillion-dollar education market", taking for granted an economic notion of education.

What is at stake here is not simply a personal point of view. Taylor has a certain (neo-liberal) understanding of the world that permeates his views on education. His discourse appears logical only under a certain conception of the world - a world view that is, just as the flat universe of the middle ages, simply wrong.

When talking about science, notions such as being, truth and meaning cannot be taken as given; they depend on the way we understand the world. For a poor psychoanalyst, science is the sublimation of sexual drives; for a Marxist, the expression of class struggle and the need to always transform capitalist society; for an american positivist, the only discourse that has any truth. By explicitly stating my philosophical position I submit to discussion not only what I want to say but also the ground on which I base myself to say something. I invite the reader to question her or his most "natural" conceptions, trying to repeat in spirit what the Greek philosophers did.

How do I understand science if I don't want to recourse to the economic discourse of innovation so dominant today? One way to do it is the proposed by the philosopher Alain Badiou [98,99]. Science as a procedure of truth - where what didn't have existence previously comes into being - formally equivalent to the artistic process, love and politics. ${ }^{13}$ This is not the place to enter in a discussion with (or concerning) Badiou; I just want to point out that the answer to what is science is not a given, and most importantly, something that cannot be reduced to the language of efficiency and performance without losing a fundamental part of it: science discovers truths.

From a certain configuration of the world, in the midst of the old worldview, science finds the new. What it was not there but that once discovered becomes something that always already was part of the world.

So, when FOM (my funding agency) writes me that "after all your primary focus is testing hypotheses, analysing research results and publishing your findings," something of vital importance gets lost. They miss the point concernig what science is. It seems that for them, reality is there waiting to be discovered by methods that we already think to know perfectly. It just needs time and funding so we can find out how the world works.

I disagree profundly with this view. I think that there is an effective act of creation in science, and more general, in naming, in giving name to

\footnotetext{
${ }^{12}$ Nature 472, 261 (2011) — doi:10.1038/472261a

${ }^{13}$ For Badiou, a "generic procedure" is the ontological process by which a truth is constituted. He insists that the human animal knows only four generic procedures: love, politics, art and science. (Alain Badiou, Logics of Worlds: Being and Event II, Albert Toscano (tr.), Continuum, 2009)
} 
things. The name is not a label that we put over things as a nameplate. When we name something, we are at the same time creating it. I do not mean a creation ex nihilo in the material world, for this would be magic. I mean that by naming, we create the way to relate to some part of reality, and in giving a name, we define the domain of possibilities for that part of reality. ${ }^{14}$

This creation associated with the act of naming does not happen in a linear way, as FOM seems to imply. It happens through events, breaks with the previously established order. Schrödinger's equation, the Copernican revolution, the theory of relativity; they are not improvements over old theories, but completely different ways of looking at the world.

There is a fruitful distinction made by the French philosopher Jacques Derrida between le futur and l'avenir, two words for "the future" in French:

In general, I try to distinguish between what one calls the future and "l'avenir." The future is that which - tomorrow, later, next century - will be. There's a future which is predictable, programmed, scheduled, foreseeable. But there is a future, l'avenir (to come) which refers to someone who comes whose arrival is totally unexpected. For me, that is the real future. That which is totally unpredictable. The other who comes without my being able to anticipate their arrival. So if there is a real future beyond this other known future, it's l'avenir in that it's the coming of the other when I am completely unable to foresee their arrival. ${ }^{15}$

The objective of science is precisely to create l'avenir. In Einstein's words: "If we knew what it was we were doing, it would not be called research." Scientists have a dream. We have no illusion about it, but we dream of discovering truths about the world. We are trying to avoid the old and we search for the new. The new that we cannot bring, that we only can hope to arrive out of grace, and for which the most subtle intelligence is needed.

Of course in doing science there is a lot of "testing hypotheses, analysing research results and publishing your findings," but this is not, nor it can be, the primary focus of my research. There is no recipe for doing science, no "best practices", no ISO 9000 that tells me how to discover something new. If there were, I could learn how to do science in 21 days with the appropiate manual and not spend four years in close contact with a senior researcher in order to learn this so particular craft.

\footnotetext{
${ }^{14}$ Think of order parameters in physics: they are not an "objective" property to be discovered by scientists, they are created insamuch as from all the possible physical observables, I pick one that signals the phase transition.

${ }^{15}$ Transcript of the film Derrida, 2002. Available at http://kirbydick.com/derrida/ DerridaTRANSCRIPT.doc
} 


\subsection{What is to be done?}

In Galileo's times, science was revolutionary. It undermined the foundations of religious institutions and consequently modified our vision of the world. Today, scientific discoveries emerge within a complex and international network of (mostly state-founded) institutions that are completely interwoven with capitalist economy. Science and capitalism seem so interrelated that people have lost the capacity to imagine or think of them as separate processes. However, science was done before the emergence of capitalism, and it will continue after capitalism disappears.

Husserl warned us, in 1935: "The crisis of European existence can end in only one of two ways: in the ruin of a Europe alienated from its rational sense of life, fallen into a barbarian hatred of spirit; or in the rebirth of Europe from the spirit of philosophy, through a heroism of reason that will definitively overcome naturalism." He didn't live to see that Europe ended up in Auschwitz and that the barbarian hatred of spirit imposed itself over the heroism of reason. Our task is not to repeat this.

Today, a certain way of understanding economy is threatening to dissolve Europe. An economic view that is not grounded on science [100]. Someone could argue that economics and politics have nothing to do with science, but I disagree for two reasons.

First, the biological understanding that we have of human beings oblige us to see ourselves not as individual units, isolated beings ${ }^{16}$. What we know from science contradicts the assumptions of economy. We are not the rational individuals that economy presupposes. It is the sum of our social relations that makes us what we are.

Second, our scientific understanding of the world obliges us to put the "care for all human beings and the natural capital upon which they depend [...] at the top of the political agenda," [101] if we want a sustainable environment in which to live. This literally means to change the world. ${ }^{17}$

Science is not an isolated activity. It depends - and at the same time shapes - the social environment in which it is made. The way we do science is both a consequence and a determining factor of the world we inhabit. To put it bluntly and stretching a bit the metaphor: the poverty of the

\footnotetext{
${ }^{16}$ For a complete treatment of this, see Alva Nöe's Out of our heads. Why you are not your brain, and other lessons from the biology of consciousness, Hill \& Wang, New York, 2009, and Evan Thompson's Mind in life: Biology, phenomenology, and the sciences of mind, Harvard University Press, 2007.

${ }^{17}$ Despite agreeing with their conclusions, I don't think that "natural capital" is a good concept. It remains too much dependent on an econiomicist view of the world, the same world view that brought us into this mess in the first place. Furthermore, people use this concept as something that is already existing on the world, and forget that by using the concept of capital we are limited to think in economical terms, which as I hope to have showed, are nothing more than blind belief.
} 
black ghettos is a necessary consequence of the existence of the University of Chicago. $^{18}$

\section{L'avenir of Science}

Amongst the four procedures that Badiou considers, science deserves a special place. Science is the only truth procedure that, in modernity, managed to bring the new inside the old institutions. On the contrary, art created the new in a constant war against the established schools, and hence the name vanguard. The same with politics: every revolution politicizes something that wasn't part of the political agenda before. However, the price science had to pay for this is that it cannot criticize the system that founds it, betraying its task in favor of the continuation of the institution of science.

Scientists do not own compliance to capitalism, for then they would be no more than intellectual prostitutes. Scientists have a debt to humanity, and even more, to truth. The opportunity of risking one's life, as Basquiat or Rimbaud did, is the price science had to pay in order to produce the new inside the old institution. However, this is not necessary, and only a circumstantial accident. Just a hundred years ago, frontier science was being done by a man working in a Swiss patent office and spending his nights reading Kant and mathematics ${ }^{19}$, and there is no reason for not to repeat his example.

People bet their lives for truth, not only Galileo, or Curie, or Spinoza, but every person who reached for unknown lands without a return ticket. Most of them died without having found anything, but their search wasn't worthless. Today, science is widely accepted as a necessary endeavor. Nobody looses his or her life for doubting the theory of relativity. However, a science that complies so gaily with the demands of society is not science at all. It is not revolutionary, it is just technique. Put somewhat differently, to make science means to search for the truth, not to be part of a community of professionals that accept without questioning the dreadful standards that governments use to assign money for research. What I mean with this is that a science that fits too easily in the established order is certainly bound not to change anything. In Derrida's terms, it belongs to le future. What we need, as should be clear by now, is a science that creates l'avenir.

I think that the problem is that we, scientists, forgot the fundamental

\footnotetext{
${ }^{18}$ This metaphor is not as far reached as it may seem at first sight. The University of Chicago was directly responsible for the application of neo-liberal policies around the globe during the 80s. This marked a shift from the full-employemnt model of John Keynes to the natural (sic.) unemployement rate of Milton Friedman. By doing this, the poverty in the black gettos was naturalised: this is the way things are.

${ }^{19}$ As recounted in Il était sept fois la révolution : Albert Einstein et les autres, Etienne Klein, Flammarion, 2007.
} 
lesson of philosophy: not to accept without question any pre-given opinion, any tradition; to search for the truth by ourselves.

The french poet Arthur Rimbaud wrote, "L'amour est à réinventer, on le sait." 20 I think that with science it is something similar. One needs to re-invent it each time. It's an adventure. And this goes not only for science. The lives of each one of us are an adventure. History is the adventure of humanity. Everything that we value and see as natural things today are not natural things, they came to be through history. We had to fight for democracy, for female vote, for equal rights for black people.

Now we have to fight for free science - the question is how?

\subsection{A ritual that is not rotten}

What I am advocating is not to leave the scientific endeavor and replace it by philosophizing. Neither to spend all our energies just talking about how to properly do science instead of go and do it. I do not have the answers on how to be a scientist, being just a starting one myself and not particularly brilliant at that.

However, I think that a rather simple linguistic exercise could help us: let's change the metaphors we use to understand science. What I propose is to look at science as something closer to love than to a private business. It would make no sense talking about relations in a couple in terms of efficiency and output - ground the decision to have a child on the GDP of the country, or find the minimum number of nights out that "produce" a good relation. In the same way, one should be embarrassed of talking about the economic impact of one's research. We do it for the fun of it (both science and love) and we should be proud of it.

The metaphor can be further extended. Relationships start with an event. A night together, a talk in a cafe, the casual encounter of our eyes in the middle of the crowd. And they continue in time, they develop. Rituals and certain ways of doing things emerge. One repeats oneself without being repetitive. A kiss remains a beautiful moment of silence, even after years of sharing life together. Rituals should be like kisses.

The inaugural event of science was the imagined talk between Socrates and Parmenides that Borges writes about. It has its own rituals and institutions, conferences and peer reviewing are two examples. But science is also different. It's a relationship that lasts thousands of years and involves millions of people. However, it has the halo of magic that love has as well. One should do science respecting that.

\footnotetext{
${ }^{20}$ Love must be reinvented, one knows it. Arthur Rimbaud, A Season in Hell, available at http://abardel.free.fr/tout_rimbaud/une_saison_en_enfer.htm
} 
Postmodernist discourses have attempted to dispose ideas as illusory. They reduce human behavior to rational decision making or hedonist pleasure satisfaction; they are cynical and cannot see beyond profit. However, these attempts are themselves mere illusion - an easy answer in a complex world, not different from the understanding of the world we had in the middle ages. To the best of our knowledge we don't know what man is. A clear statement of the limits of what we know is much greater aid than faulty and simplistic models, mostly when they become normative, as it is the case with neo-classical economics. Furthermore, by acknowledging our ignorance, we open the space for our freedom to exist. The freedom to do what we want.

\subsection{Conclusion: The science I want to make}

I was taken out of the house surrounded by shit thanks to the knowledge that was waiting for me in the Encyclopedia. I had no future, and knowledge gave me an avenir. If I want to understand my personal history I cannot avoid to confront the political and social history of Chile, and in general, of the world. And since biologically we are not isolated individuals, but part of a network of relations, my story is also part of your story. This story started with two men in Greece who decided to think, continued with all those who kept the conversation alive through history, and is still written today.

I want a science that doesn't stay unabated by social reality. A science that remains faithful to the event of philosophy. A science that refuses to accept without question any pre-given opinion, any tradition, and so looks for the truth in itself, with the certitude that discussion is the not-impossible path to reach it. A science that is truly interdisciplinary, even at the cost of not publishing several papers a year but instead each time that a new and big understanding is developed, may this take years. A science with a critical spirit and not afraid of telling the truth; and brave enough to act accordingly.

Wittgenstein writes somewhere in his notebooks that "each of the sentences I write is trying to say the whole thing, i.e., the same thing over and over again; it is as though they were all simply views of one object seen from different angles." [102] For me, this also applies to science. We are trying to understand the same thing from different points of view: the mystery of existence; the phenomenon of life; that things are; in a word, we are trying to understand the world. And this should be science's goal; not the number of publications, the economic impact of research or the fancy of funding agencies or managers. My research should reflect this. 



\section{Chapter 7}

\section{Conclusions and Outlook}

In this thesis, I have studied different systems with both hard- and softspheres models. In particular, I studied how the long range interaction affected the cooling behaviour of granular gases; developed a new algorithm for the cluster-cluster aggregation process; and discovered a new mechanism to control the axial segregation in rotating tumblers by the shaping the container in a particular way.

General conclusions include:

1. The dynamics of 2D free cooling hard sphere gas in the dense regime is better predicted by a modified pair correlation function at contact that considers the phase transition to ordered crystals.

2. Discrete potentials reproduce much of the physics of continuous potentials, at least for the free cooling of granular gases at low densities.

3. The fractal dimension of granular aggregates obtained by cluster-cluster aggregation depends on the density of the homogeneous initial gas.

4. In the presence of long range forces, cluster formation in granular gases is a complex phenomenon that involves the subtle interplay between dissipation and attractive force.

5. I have developed a framework that comprises an easily modifiable experimental set-up and same-scale computer simulations for rotating tumblers.

6. There are fundamental differences between the flow in a convex and a concave tumbler. By controlling the shape of the drum, it is possible to produce different dynamics with a single driving frequency.

7. Axial segregation in rotating tumblers can be controlled by modifying the shape of the drum. This can have far fetching practical consequences in industries ranging from pharmaceutical to mining. 
In the different subjects we studied there are a few lines of research that could be continued. In what follows we give an account of the ones we consider the most important.

\section{Different Models for Clustering}

One of the open questions left by this thesis is what is the relation between the clusters obtained by the "sticky" potential and the square well.

One is extremely simple but does have the proper physics. For example, the role of rotation is totally neglected; Does this modify the structure of the clusters? How bad is this approximation for dilute systems? How does the inclusion of rotation - and hence conservation of angular momentum affects the cooling dynamics?

Finally, with the creation of a new stable ED algorithm by Bannerman [103] it is possible now to simulate non-spherical particles in a stable way. The extension to arbitrary clusters is rather straightforward, and an obvious line of research to be applied, among other, in the simulation of nano-cluster in a fluidised bed.

\section{Event-driven simulations}

As future work, one can envisage at least three areas of development. First, finish the implementation of aggregates of spherical particles in event-driven simulations. This could be use to, for example, continue the cluster-cluster aggregation process in 3D including rotation, so to study the dynamics of nano-aggregates in gases, or take into account the duration of collisions in event-driven simulations (see Ref. [104]). Furthermore, event-driven algorithms could be used to study the dynamics of systems where Monte-Carlo simulations have already showed fascinating properties [105].

Second, study of segregation in long rotating drums. For this, the use of sleeping particles would be extremely efficient, allowing the simulation of millions of particles at almost no computational cost. To realize this, a proper comparison between event-driven simulations and experiments is needed, in order to assert the parameter region where the model is representative of the actual dynamics.

Third, continue the study of the discontinuous potentials as approximations of long range potentials. Discontinuous potentials has been already used in the simulation of complex molecules as benzene [106], or in the simulation of polymer chains [107]. To quantify the applicability range of discrete potentials for long-range interactions systems can improve the understanding of complex dynamics with a relatively simple simulation. 


\section{Shaping Segregation}

Our results show that by controlling the shape of a rotating tumbler, the segregation in the axial direction can be controlled.

The next step should be to contact industrial partners to see how and where this knowledge can be used, for example, in SAG mills where the biggest problem concerns the milling of the medium size particles. Together with this, a more comprehensive study involving different drum shapes, different component number, different symmetries, and why not, non-symmetric shapes, is the eviden way of continuation for this work.

In concrete, I would like to answer the following questions: What is the optimal drum's shape to obtain a given segregation pattern? How to use this insight in existing processes in the granular industry? What is the best shape to maximise milling volume and minimise residency time in a SAG mill? 


\section{Curriculum vitae}

\section{Sebastián González}

Born on July 29th, 1983 in Santiago, Chile

Jun $2001 \quad$ Bachelor of Science in Physics

University of Chile

Dec 2010 Master of Science in Physics,

University of Chile

Jan. 2010 - Jan. 2013 PhD candidate,

University of Twente

Publications

1. S. González, D. Risso, and R. Soto. Extended event driven molecular dynamics for simulating dense granular matter. The European Physical Journal - Special Topics, 179:33-41, 2009. 10.1140/epjst/e2010-01192-4

2. S. González and S. Luding. Consequences of using different paircorrelation functions on the stability properties of the homogeneous cooling state for a monodisperse system of near-elastic disks. Eur. Phys. J. Special Topics, 179:55-68, 2009

3. S. González, A. R. Thornton, and S. Luding. An event-driven algorithm for fractal cluster formation. Computer Physics Communications, 182(9):1842 - 1845, 2011 


\section{Bibliography}

[1] J. L. Borges. Labyrinths: Selected Stories \& Other Writings. New Directions Paperbook, 186. New Directions Pub, 1964.

[2] P. G. de Gennes. Reflections on the mechanics of granular matter. Physica A, 261:267-293, 1998.

[3] H. M. Jaeger, S. R. Nagel, and R. P. Behringer. Granular solids, liquids, and gases. Rev. Mod. Phys., 68(4):1259-1273, 1996.

[4] J. Mellmann. The transverse motion of solids in rotating cylindersforms of motion and transition behavior. Powder Technology, 118(3):251 $270,2001$.

[5] G. Seiden and P. J. Thomas. Complexity, segregation, and pattern formation in rotating-drum flows. Rev. Mod. Phys., 83:1323-1365, Nov 2011.

[6] J. M. Ottino and D. V. Khakhar. Mixing and segregation of granular materials. Ann. Rev. Fluid Mech., 32:55, 2000.

[7] K. M. Hill and J. Kakalios. Reversible axial segregation of binary mixtures of granular materials. Phys. Rev. E, 49(5):R3610, 1994.

[8] T. Pöschel and N. V. Brilliantov, editors. Granular Gas Dynamics. Springer, Berlin, Germany, 2003.

[9] B. D. Lubachevsky. How to simulate billards and similar systems. J. Comp. Phys., 94(2):255, 1991.

[10] T. Pöschel and T. Schwager. Computational Granular Dynamics. Springer, Berlin, 2005.

[11] M. Marín, D. Risso, and P. Cordero. Efficient algorithms for many-body hard particle molecular dynamics. Journal of Computational Physics, 109(2):306 - 317, 1993. 
[12] S. González, D. Risso, and R. Soto. Extended event driven molecular dynamics for simulating dense granular matter. The European Physical Journal - Special Topics, 179:33-41, 2009. 10.1140/epjst/e2010-011924.

[13] M. N. Bannerman, R. Sargant, and L. Lue. Dynamo: a free 2(n) general event-driven molecular dynamics simulator. Journal of Computational Chemistry, 32(15):3329-3338, 2011.

[14] A. R. Thornton, T. Weinhart, S. Luding, and O. Bokhove. Modeling of particle size segregation: Calibration using the discrete particle method. International Journal of Modern Physics C, 23(08):1240014, 2012 .

[15] S. Luding. Collisions \& contacts between two particles. In H. J. Herrmann, J.-P. Hovi, and S. Luding, editors, Physics of dry granular media - NATO ASI Series E350, page 285, Dordrecht, 1998. Kluwer Academic Publishers.

[16] V. Ogarko and S. Luding. A fast multilevel algorithm for contact detection of arbitrarily polydisperse objects. Computer Physics Communications, 183(4):931-936, 2012.

[17] P. K. Haff. Grain flow as a fluid-mechanical phenomenon. J. Fluid Mech., 134:401-430, 1983.

[18] I. Goldhirsch and G. Zanetti. Clustering instability in dissipative gases. Phys. Rev. Lett., 70(11):1619-1622, 1993.

[19] S. McNamara. Hydrodynamic modes of a uniform granular medium. Phys. Fluids A, 5(12):3056 - 3070, 1993.

[20] S. McNamara and W. R. Young. Dynamics of a freely evolving, twodimensional granular medium. Phys. Rev. E, 53(5):5089-5100, 1996.

[21] H. J. Herrmann, J.-P. Hovi, and S. Luding, editors. Physics of dry granular media - NATO ASI Series E 350, Dordrecht, 1998. Kluwer Academic Publishers.

[22] S. Luding and H. J. Herrmann. Cluster growth in freely cooling granular media. Chaos, 9(3):673-681, 1999.

[23] T. Pöschel and S. Luding, editors. Granular Gases, Berlin, 2001. Springer. Lecture Notes in Physics 564.

[24] J. J. Brey, M. J. Ruiz-Montero, and A. Dominguez. Shear state of freely evolving granular gases. Phys. Rev. E, 78(4):041301, 2008. 
[25] P. Richard, A. Valance, J. F. Metayer, P. Sanchez, J. Crassous, M. Louge, and R. Delannay. Rheology of confined granular flows: Scale invariance, glass transition, and friction weakening. Phys. Rev. Lett., 101(24):248002, 2008.

[26] E. Khain. Bistability and hysteresis in dense shear granular flow. Europhys. Lett., 87:14001, 2009.

[27] T. Shinbrot, N. H. Duong, M. Hettenbach, and L. Kwan. Coexisting static and flowing regions in a centrifuging granular heap. Granular Matter, 9(5):295-307, 2007.

[28] H. P. Zhu, Z. Y. Zhou, R. Y. Yang, and A. B. Yu. Discrete particle simulation of particulate systems: A review of major applications and findings. Chem. Eng. Science, 63(23):5728-5770, 2008.

[29] I. Goldhirsch, M. L. Tan, and G. Zanetti. A molecular dynamical study of granular fluids I: The unforced granular gas in two dimensions. Journal of Scientific Computing, 8:1-40, 1993.

[30] J. J. Brey, M. J. Ruiz-Montero, and D. Cubero. Homogeneous cooling state of a low-density granular flow. Phys. Rev. E, 54:3664-3671, 1996.

[31] S. Luding. Clustering instabilities, arching, and anomalous interaction probabilities as examples for cooperative phenomena in dry granular media. T.A.S.K. Quarterly, Scientific Bulletin of Academic Computer Centre of the Technical University of Gdansk, 2(3):417-443, July, 1998.

[32] S. Luding and S. McNamara. How to handle the inelastic collapse of a dissipative hard-sphere gas with the TC model. Granular Matter, 1(3):113-128, 1998. e-print cond-mat/9810009.

[33] S. Miller and S. Luding. Cluster growth in two- and three-dimensional granular gases. Phys. Rev. E, 69:031305, 2004.

[34] S. Luding. Structure and cluster formation in granular media. Pramana-Journal of Physics, 64(6):893-902, 2005.

[35] D. Bonamy, F. Daviaud, L. Laurent, M. Bonetti, and J. P. Bouchaud. Multiscale clustering in granular surface flows. Phys. Rev. Lett., 89(3):034301, 2002.

[36] N. Sela and I. Goldhirsch. Hydrodynamic equations for rapid flows of smooth inelastic spheres to Burnett order. J. Fluid Mech., 361:41-74, 1998. 
[37] S. Luding. Towards dense, realistic granular media in 2d. Nonlinearity, 22(12):R101-R146, 2009.

[38] J. T. Jenkins and M. W. Richman. Kinetic theory for plane shear flows of a dense gas of identical, rough, inelastic, circular disks. Phys. of Fluids, 28:3485-3494, 1985.

[39] I. S. Aranson and L. S. Tsimring. Patterns and collective behavior in granular media: Theoretical concepts. Rev. Mod. Phys., 78(2):641-692, 2006 .

[40] K. Saitoh and H. Hayakawa. Rheology of a granular gas under a plane shear. Phys. Rev. E, 75(2):021302, 2007.

[41] E. Khain. Hydrodynamics of fluid-solid coexistence in dense shear granular flow. Phys. Rev. E, 75(5):051310, 2007.

[42] D. Henderson. A simple equation of state for hard discs. Molec. Phys., 30(3):971-972, 1975.

[43] E. L. Grossman, T. Zhou, and E. Ben-Naim. Towards granular hydrodynamics in two-dimensions. Phys. Rev. E, 55:4200, 1997.

[44] S. Luding. Global equation of state of two-dimensional hard sphere systems. Phys. Rev. E, 63:042201-1-4, 2001.

[45] C. Dominik and A. G. G. Tielens. The physics of dust coagulation and the structure of dust aggregates in space. The Astrophysical Journal, 480:647-673, 1997.

[46] J. R. Royer, D. J. Evans, L. Oyarte, Q. Guo, E. Kapit, M. E. Mobius, S. R. Waitukaitis, and H. M. Jaeger. High-speed tracking of rupture and clustering in freely falling granular streams. Nature, 459(7250):11101113, Jun 2009.

[47] M. Moseler and U. Landman. Formation, stability, and breakup of nanojets. Science, 289(5482):1165-1169, 2000.

[48] A. S. Keys, A. R. Abate, S. C. Glotzer, and D. J. Durian. Measurement of growing dynamical length scales and prediction of the jamming transition in a granular material. Nat Phys, 3(4):260-264, Apr 2007.

[49] A. Schmidt-Ott. In situ measurement of the fractal dimensionality of ultrafine aerosol particles. Applied Physics Letters, 52(12):954-956, 1988. 
[50] T. A. Witten and L. M. Sander. Diffusion-limited aggregation. Phys. Rev. B, 27:5686-5697, May 1983.

[51] E. Trizac and J. P. Hansen. Dynamic scaling behavior of ballistic coalescence. Phys. Rev. Lett., 74(21):4114-4117, 1995.

[52] P. Meakin and Z. R. Wasserman. Some universality properties associated with the cluster-cluster aggregation model. Physics Letters A, 103(67):337 - 341, 1984.

[53] J. H. Werth, H. Knudsen, and H. Hinrichsen. Agglomeration of oppositely charged particles in nonpolar liquids. Phys. Rev. E, 73:021402, Feb 2006.

[54] L. Hernández de la Peña, R. van Zon, J. Schofield, and S. B. Opps. Discontinuous molecular dynamics for semiflexible and rigid bodies. The Journal of Chemical Physics, 126(7):074105, 2007.

[55] C. De Michele. Simulating hard rigid bodies. Journal of Computational Physics, 229(9):3276 - 3294, 2010.

[56] G. F. Carnevale, Y. Pomeau, and W. R. Young. Statistics of ballistic agglomeration. Phys. Rev. Lett., 64:2913-2916, Jun 1990.

[57] M. N. Bannerman, J. E. Kollmer, A. Sack, M. Heckel, P. Mueller, and T. Pöschel. Movers and shakers: Granular damping in microgravity. Phys. Rev. E, 84:011301, Jul 2011.

[58] J. S. Olafsen and J. S. Urbach. Velocity distributions and density fluctuations in a 2D granular gas. Phys. Rev. E, 60:R2468, 1999.

[59] S. McNamara and W. R. Young. Kinetics of a one-dimensional granular medium in the quasielastic limit. Phys. Fluids A, 5(1):34, 1993.

[60] N. V. Brilliantov and T. Pöschel. Deviation from maxwell distribution in granular gases with constant restitution coefficient. Phys. Rev. E, 61:2809-2814, 2000.

[61] N. Brilliantov and T. Pöschel. Hydrodynamics and transport coefficients for dilute granular gases. Physical Review E, 67:061304, 2003. e-print cond-mat/0301152.

[62] S. E. Esipov and T. Pöschel. The granular phase diagram. J. Stat. Phys., 86:1385-1395, 1997. e-print cond-mat/9609096.

[63] T. Pahtz, H. J. Herrmann, and T. Shinbrot. Why do particle clouds generate electric charges? Nat Phys, 6(5):364-368, May 2010. 
[64] M. Horanyi, G. Morfill, and E. Grun. Mechanism for the acceleration and ejection of dust grains from Jupiter's magnetosphere. Nature, 363(6425):144-146, May 1993.

[65] A. Brahic. Numerical simulation of a system of colliding bodies in a gravitational field. Journal of Computational Physics, 22(2):171 - 188, 1976.

[66] F. G. Bridges, A. Hatzes, and D. N. C. Lin. Structure, stability and evolution of Saturn's rings. Nature, 309:333, 1984.

[67] W. F. Carnahan and K. E. Starling. Equation of state for nonattracting rigid spheres. J. Chem. Phys., 51(2):635-636, 1969.

[68] S. Torquato. Nearest-neighbor statistics for packings of hard spheres and disks. Phys. Rev. E, 51:3170, 1995.

[69] V. Ogarko and S. Luding. Equation of state and jamming density for equivalent bi- and polydisperse, smooth, hard sphere systems. The Journal of Chemical Physics, 136(12):124508, 2012.

[70] S. González and S. Luding. Consequences of using different paircorrelation functions on the stability properties of the homogeneous cooling state for a monodisperse system of near-elastic disks. Eur. Phys. J. Special Topics, 179:55-68, 2009.

[71] T. Pöschel, N. V. Brilliantov, and T. Schwager. Transient clusters in granular gases. J. Phys.-Cond. Matter, 17(24):S2705-S2713, 2005.

[72] M. K. Müller and S. Luding. Homogeneous cooling with repulsive and attractive long-range potentials. Mathematical Modelling of Natural Phenomena, 6(04):118-150, Jul 2011.

[73] M. K. Müller and S. Luding. Homogeneous cooling with repulsive and attractive long-range interactions. AIP Conference Proceedings, 1145(1):697-700, 2009.

[74] N. Brilliantov, C. Salueña, T. Schwager, and T. Pöschel. Transient structures in a granular gas. Phys. Rev. Lett., 93:134301, Sep 2004.

[75] S. González, A. R. Thornton, and S. Luding. An event-driven algorithm for fractal cluster formation. Computer Physics Communications, 182(9):1842 - 1845, 2011.

[76] N. G. van Kampen. Condensation of a classical gas with long-range attraction. Phys. Rev., 135(2A):A362-A369, Jul 1964. 
[77] V. Y. Zaburdaev, M. Brinkmann, and S. Herminghaus. Free cooling of the one-dimensional wet granular gas. Phys. Rev. Lett., 97:018001, Jul 2006.

[78] S. Ulrich, T. Aspelmeier, K. Roeller, A. Fingerle, S. Herminghaus, and A. Zippelius. Cooling and aggregation in wet granulates. Phys. Rev. Lett., 102:148002, Apr 2009.

[79] L. de Martin, W. G. Bouwman, and J. R. van Ommen. Two-level hierarchical structure in nano-powder agglomerates in gas media. Bulletin of the American Physical Society, 57, 2012.

[80] S. W. Meier, S. E. Cisar, R. M. Lueptow, and J. M. Ottino. Capturing patterns and symmetries in chaotic granular flow. Phys. Rev. E, 74:031310, Sep 2006.

[81] I. C. Christov, J. M. Ottino, and R. M. Lueptow. Chaotic mixing via streamline jumping in quasi-two-dimensional tumbled granular flows. Chaos: An Interdisciplinary Journal of Nonlinear Science, 20(2):023102, 2010.

[82] K. M. Hill, N. Jain, and J. M. Ottino. Modes of granular segregation in a noncircular rotating cylinder. Phys. Rev. E, 64:011302, Jun 2001.

[83] L. Naji and R. Stannarius. Axial and radial segregation of granular mixtures in a rotating spherical container. Phys. Rev. E, 79:031307, Mar 2009.

[84] N. A. Pohlman and D. F. Paprocki Jr. Transient behavior of granular materials as result of tumbler shape and orientation effects. Granular Matter, pages 1-9, 2012.

[85] G. Metcalfe, T. Shinbrot, J. J. McCarthy, and J. M. Ottino. Avalanche mixing of granular solids. Nature, 374(6517):39-41, Mar 1995.

[86] P. W. Cleary, R. Morrisson, and S. Morrell. Comparison of DEM and experiment for a scale model SAG mill. International Journal of Mineral Processing, 68(14):129 - 165, 2003.

[87] D. Morton and S. Dunstall. Using the Web to increase the availability of DEM-based mill modelling. Minerals Engineering, 17(1112):1199 1207, 2004.

[88] D. Mounty. Particle Size Segregation in Convex Rotating Drums. PhD thesis, University of Manchester, 2007. 
[89] J. M. N. T. Gray. Granular flow in partially filled slowly rotating drums. Journal of Fluid Mechanics, 441:1-29, 72001.

[90] M. B. Hu, X. Z. Kong, Q. S. Wu, and Y. H. Wu. Effects of container geometry on granular segregation pattern. Chinese Physics B, 14(9):1844, 2005.

[91] M. B. Hu, X. Z. Kong, Q. S. Wu, and Y. H. Wu. Granular segregation in a multi-botleneck container: Mobility effect. International Journal of Modern Physics B, 19(10):1793-1800, 2005.

[92] D. Shi, A. A. Abatan, W. L. Vargas, and J. J. McCarthy. Eliminating segregation in free-surface flows of particles. Phys. Rev. Lett., 99:148001, Oct 2007 .

[93] S. B. Savage and C. K. K. Lun. Particle size segregation in inclined chute flow of dry cohesionless granular solids. J. Fluid. Mech., 189:311, 1988.

[94] O. Zik, D. Levine, S. G. Lipson, S. Shtrikman, and J. Stavans. Rotationally induced segregation of granular materials. Phys. Rev. Lett., 73:644-647, Aug 1994.

[95] M. M. H. D. Arntz, W. K. Otter, H. H. Beeftink, R. M. Boom, and W. J. Briels. The influence of end walls on the segregation pattern in a horizontal rotating drum. Granular Matter, pages 1-14, 2012.

[96] K. Karatani. Beyond capital-nation-state. Rethinking Marxism, 20(4):569-595, 2008.

[97] M. Buchanan. Pattern of growth. Nature Physics, 8(11):775-775, 2012.

[98] A. Badiou and O. Feltham. Being and Event. Continuum, 2007.

[99] A. Badiou. Logics of Worlds: Being and Event, 2. Being and Event. Continuum, 2009.

[100] J. P. Bouchaud. Economics needs a scientific revolution. Nature, 455(7217):1181-1181, Oct 2008.

[101] P. R. Ehrlich, P. M. Kareiva, and G. C. Daily. Securing natural capital and expanding equity to rescale civilization. Nature, 486(7401):68-73, Jun 2012.

[102] L. Wittgenstein, G. H. Wright, and H. Nyman. Culture and Value. University of Chicago Press, 1984. 
[103] M. N. Bannerman and S. Strobl. Stable Event-Driven Particle Dynamics: Spherically Symmetric Potentials. ArXiv e-prints, November 2012.

[104] P. Müller and T. Pöschel. Oblique impact of frictionless spheres: on the limitations of hard sphere models for granular dynamics. Granular Matter, pages 1-6, 2012. 10.1007/s10035-012-0324-5.

[105] J. de Graaf, R. van Roij, and M. Dijkstra. Dense regular packings of irregular nonconvex particles. Phys. Rev. Lett., 107:155501, Oct 2011.

[106] L. Hernández de la Peña, R. van Zon, J. Schofield, and S. B. Opps. Discontinuous molecular dynamics for rigid bodies: Applications. The Journal of Chemical Physics, 126(7):074106, 2007.

[107] C. Hartmann, C. Schütte, G. Kalibaeva, M. Di Pierro, and G. Ciccotti. Fast simulation of polymer chains. The Journal of Chemical Physics, 130(14):144101, 2009. 